JOSÉ AMENDOLA NETTO ANDRADE

\title{
REINFORCEMENT LEARNING APPLIED TO VESSEL NAVIGATION IN FAST-TIME SIMULATIONS
}


JOSÉ AMENDOLA NETTO ANDRADE

\title{
REINFORCEMENT LEARNING APPLIED TO VESSEL NAVIGATION IN FAST-TIME SIMULATIONS
}

\author{
Master Thesis presented to the Escola \\ Politécnica, Universidade de São Paulo to \\ obtain the degree of Master of Science.
}




\section{REINFORCEMENT LEARNING APPLIED TO VESSEL NAVIGATION IN FAST-TIME SIMULATIONS}

Revised Version

Master Thesis presented to the Escola Politécnica, Universidade de São Paulo to obtain the degree of Master of Science.

Research Area:

Control and Automation

Advisor:

Prof. Dr. Eduardo Aoun Tannuri

Co-advisor:

Prof. Dr. Fábio Gagliardi Cozman 
Autorizo a reprodução e divulgação total ou parcial deste trabalho, por qualquer meio convencional ou eletrônico, para fins de estudo e pesquisa, desde que citada a fonte.

Este exemplar foi revisado e corrigido em relação à versão original, sob responsabilidade única do autor e com a anuência de seu orientador.

São Paulo, de de

Assinatura do autor:

Assinatura do orientador:

\section{Catalogação-na-publicação}

Amendola, José

Reinforcement Learning Applied to Vessel Navigation in Fast-Time Simulations / J. Amendola -- versão corr. -- São Paulo, 2020.

$96 \mathrm{p}$.

Dissertação (Mestrado) - Escola Politécnica da Universidade de São Paulo. Departamento de Engenharia Mecatrônica e de Sistemas Mecânicos.

1.Reinforcement Learning 2.Navigation in Restricted Waters 3.Machine Learning 4.Fast-Time Simulations I.Universidade de São Paulo. Escola Politécnica. Departamento de Engenharia Mecatrônica e de Sistemas Mecânicos II.t. 


\section{ACKNOWLEDGMENTS}

I am grateful to my family for always supporting me and encouraging me in difficult times. Specially my mother Regiane, for making efforts so as to provide me with resources and opportunities she had not had herself. I would like to thank my fiancee Juliana for all the love, patience and understanding during the conclusion of this work.

I am very grateful to my advisor, Professor Eduardo Tannuri, who encouraged me and promptly endorsed my idea of combining reinforcement learning with maritime simulation. He was was very open to conversation, teached me a lot about the simulation domain and provided resources for the progress of the work whenever I needed. I am also grateful to my co-advisor, Professor Fabio Cozman, for guidance during publications and work development. A special thanks goes to Professor Anna Reali, who was very dedicated in counseling me and using her expertise to improve my work.

I am grateful to all TPN members for creating a light and fruitful work atmosphere, always indulging in collaboration and love for knowledge. Thanks to Humberto Makiyama, for being a comprehensive leader and pushing me trough professional enhancement. Thanks to all my colleagues Leonardo Garcia, Felipe Marino, Asdrubal Queiroz, Leanderson Ribeiro, Edgar Szilagy, for the many inspiring conversations. Thanks to Lucas Miura for the invaluable help and technical discussions. Thanks to Hélio Sinohara and André Ianagui for the willingness to answer questions.

Thanks to Petrobrás, who has funded the TPN laboratory and made possible a high quality research environment. The TPN laboratory has been inspiring me since I was an undergraduate at the University of São Paulo and I am proud to be part of it. Finally, I would like to thank and acknowledge CnPQ for its scholarship. 
"To know that we know what we know, and to know that we do not know what we do not know, that is true knowledge."

-- Nicolaus Copernicus 


\section{RESUMO}

Simulações em tempo acelerado têm se provado uma ferramenta essencial para engenharia marítima, não somente para projeto de navios, mas também para detectar pontos críticos e possíveis gargalos em projetos de portos. Contudo, tais simulações não são realizadas por pilotos profissionais e isso pode se tornar uma tarefa complexa com resultados não tão fiéis à realidade. Tais questões podem apresentar uma oportunidade para introduzir Aprendizado por Reforço no domínio marítimo.

Esse trabalho propõe uma solução baseada em Aprendizagem por Reforço que é capaz de gerar de forma automática trajetórias de navios em águas restritas sob o efeito de forças ambientais. O agente aprende interagindo com o simulador e recebendo sinais de reforço. Ele também provê comandos discretos em intervalos discretos de tempo para emular as limitações presentes na pilotagem humana.

O método avalia a versão distribuída de dois algoritmos no estado da arte em aprendizado por reforço. Ele lida com segmentos de canais como episódios separados e inclui informação de curvatura para ações antecipatórias. Experimentos foram conduzidos considerando cenários realistas com canais estreitos e curvos onde a incidência de vento e corrente variam ao longo da trajetória.

O caráter inovador do trabalho se dá pelo fato de que a solução proposta não requer qualquer conhecimento prévio dos modelos dinâmicos ou de caminhos pré-definidos para serem seguidos pelo navio. Isso pode impactar as simulações em tempo acelerado exigindo menos esforço humano na obtenção das trajetórias. O método adotado utiliza uma representação simples e pode ser aplicado a quaisquer canais que respeitem as regulamentações técnicas locais.

Palavras-Chave - Aprendizado por Reforço; Navegação em águas restritas; Simulações em tempo acelerado 


\section{ABSTRACT}

Fast-time simulations have been proven to be an essential tool for maritime engineering, not only in ship design but also by detecting critical situations and bottlenecks in projects of ports. However, such simulations are not performed by professional pilots and might become a complex task with results not so close to reality. Such issues can present an opportunity for introducing Reinforcement Learning methods in the maritime domain.

This work proposes a Reinforcement Learning based solution which is able to automatically generate vessel trajectories in restricted waters under the effect of environment forces. The agent learns by interacting with the simulator and receiving reward signals. It also gives discrete commands in spaced time steps in order to emulate limitations of human piloting.

The method evaluates the distributed version of two state-of-art Reinforcement Learning algorithms. It handles channel segments as separate episodes and includes curvature information for anticipating actions. Experiments were run considering realistic scenarios with narrow curved channels where wind and current incidence varies along the trajectory.

The novelty of the work is the fact that the solution proposed requires no prior knowledge on dynamic models or predefined line paths to be followed by the ship. It may impact in fast-time simulations by requiring less human effort in trajectories generation. The method adopted keeps a simple representation and can be applied to any port channel configuration that respects local technical regulations.

Keywords - Reinforcement Learning; Navigation in Restricted Waters; Fast-Time Simulations 


\section{LIST OF FIGURES}

1 Rudder and propeller of a vessel . . . . . . . . . . . . . . . . . . . 22

2 Vessel coordinate system . . . . . . . . . . . . . . . . . . 23

3 Forces on the rudder . . . . . . . . . . . . . . . . . . 24

$4 \quad$ RL basic scheme . . . . . . . . . . . . . . . . . 25

5 Policy Iteration . . . . . . . . . . . . . . . . . . . 30

6 Route planning for areas discretized in grids . . . . . . . . . . . . . . . . 34

7 Line of Sight Technique and set-point error measurements . . . . . . . . . 35

8 State variables $($ in bold) . . . . . . . . . . . . . . . . . 45

9 Top: a compact representation with no information about vessel's position may "confuse" the policy. Bottom: Vessel's position included in the state representation prevents the reuse of policy in different parts of the channel. 47

10 Channel partitions. . . . . . . . . . . . . . . . . . . 48

11 Symmetry of experiences for pair of segments . . . . . . . . . . . . . . . 49

12 Effects of rate_of_turn punishment; $K=1$ for Less Punitive Behavior; $K=10$ for Punitive Behavior (adopted value) . . . . . . . . . . . . . 52

13 Architecture: APE-X-DQN . . . . . . . . . . . . . . . 53

14 Neural network architecture for APE-X-DQN policy . . . . . . . . . . . . . 55

15 Neural network architecture for PPO policy . . . . . . . . . . . . . 58

16 Suezmax DP tanker: (left) main characteristics; (right) maneuvering properties. . . . . . . . . . . . . . . . . . . . 62

17 Channel topology generated for training . . . . . . . . . . . . 63

18 Average accumulated reward per episode (moving average from 100 episodes). Mean from 3 learning trials in darker orange and darker blue; Standard deviation in shaded lighter orange and lighter blue. . . . . . . . . . . . . . . . 65 
19 Average episode length (moving average from 100 episodes). Mean from 3 learning trials in darker orange and darker blue; Standard deviation in shaded lighter orange and lighter blue. . . . . . . . . . . . . . . . . . .

20 Fraction of channel reached by periodic evaluation of policy. Mean from 3 learning trials in darker orange and darker blue; Standard deviation in shaded lighter orange and lighter blue. . . . . . . . . . . . . . . 66

21 Location of the Porto Sudeste Access Channel. . . . . . . . . . . . . . . . . 67

22 Porto Sudeste Access Channel over the Nautical Chart. . . . . . . . . . . . 67

23 Environmental conditions - Current Vectors and Wind Direction: (up) Flood tide scenario; (down) Ebb tide scenario. . . . . . . . . . . . . . 68

24 Trajectories for policy trained with APE-X-DQN. Blue ship: Scenario E04; Orange ship: Scenario E05; Purple ship: E08, Green ship: E10. . . . . . . 69

25 Vessel's heading and course over ground in Scenario F05 for APE-X-DQN . 70

26 Vessel's heading and course over ground in Scenario F05 for PPO . . . . . 70

27 Rudder angles commanded by the agent and its effective value in Scenario F05 for APE-X-DQN . . . . . . . . . . . . . . . . . . . . 71

28 Rudder angles commanded by the agent and its effective value in Scenario $\mathrm{F} 05$ for $\mathrm{PPO} \ldots \ldots \ldots \ldots \ldots$. . . . . . . . . . . . . . . . . . . . . . . . . . . . .

29 Speed over ground along the navigation in Scenario F05 using APE-X-DQN. 72

30 Speed over ground along the navigation in Scenario F05 using PPO. . . . . 72

31 Distance to margins at Porto Sudeste for scenarios using APE-X-DQN. 0-F04, 1-F05, 2-F08, 3-F10, 4-E04, 5-E05, 6-E08, 7-E10 . . . . . . 73

32 Distance to margins at Porto Sudeste for scenarios using PPO. 0-F04, 1F05, 2-F08, 3-F10, 4-E04, 5-E05, 6-E08, 7-E10 . . . . . . . . 74

33 Location of Suape Access Channel . . . . . . . . . . . . . . . . . . . . . . 75

34 Suape Access Channel over the Nautical Chart . . . . . . . . . . . . . . . . 75

35 Visual representation of wind and current conditions evaluated in Suape Channel.A- N04W20, B-N08W20, C-N08W24, D-N08W20-330 . . . . . . . 76 
36 Trajectories for policy trained with APE-X-DQN. Blue ship: Scenario N04W20; Orange ship: Scenario N08W20-330; Purple ship:Scenario N08W20

, Green ship: Scenario N08W24. . . . . . . . . . . . . . . . . . . 77

37 Vessel's heading and course over ground in Scenario N08W20 for APE-X-

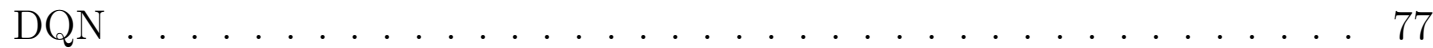

38 Vessel's heading and course over ground in Scenario N08W20 for PPO . . . 78

39 Rudder angles commanded by the agent and its effective value in Scenario N08W20 for APE-X-DQN . . . . . . . . . . . . . . . . 78

40 Rudder angles commanded by the agent and its effective value in Scenario N08W20 for PPO . . . . . . . . . . . . . . . . . . . 79

41 Speed over ground along the navigation in Scenario F05 using APE-X-DQN. 79

42 Speed over ground along the navigation in Scenario F05 using PPO. . . . . 80

43 Distance to margins for scenarios using APE-X-DQN. 0-N04W20, 1-N08W20, 2-N08W24, 3-N08W20-330. . . . . . . . . . . . . . 80

44 Distance to margins for scenarios using PPO. 0-N04W20, 1-N08W20, 2N08W24, 3-N08W20-330. . . . . . . . . . . . . . . . 81

45 Vessel's heading and course over ground in Scenario F08 for APE-X-DQN training in Porto Sudeste . . . . . . . . . . . . . . . . . . . . . . . 82

46 Distance to margins in Scenario F08 for APE-X-DQN training in Porto

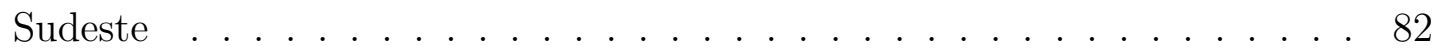

47 Neural network . . . . . . . . . . . . . . . . . . . 87

48 Neuron basic scheme . . . . . . . . . . . . . . . . . . . . . . 88

49 Sigmoidal function . . . . . . . . . . . . . . . . . . . . . . . . . 89

$50 \quad$ Forward propagation and Backpropagation . . . . . . . . . . . . . . . 90 


\section{LIST OF TABLES}

1 Reviewed RL applications to maritime navigation . . . . . . . . . . 38

3 State variables. . . . . . . . . . . . . . . . . . . 44

4 Dividing Factors. . . . . . . . . . . . . . . . . 46

5 Environmental scenarios evaluated in Porto Sudeste. . . . . . . . . . . 68

6 Environmental scenarios evaluated in Suape. . . . . . . . . . . . . . 76 


\section{ACRONYMS}

ANN Artificial Neural Network.

DQN Deep Q Network.

FTS Fast-Time Simulations.

MDP Markov Decision Processes.

PG Policy Gradient.

PPO Proximal Policy Optimization.

RL Reinforcement Learning.

RTS Real-Time Simulations.

SMS Ship Maneuvering Simulators.

TD Temporal Difference. 


\section{LIST OF SYMBOLS}

$\alpha \quad$ Learning rate for $\mathrm{Q}$ learning

$\alpha_{p r} \quad$ APE-X-DQN Prioritization factor

$\beta \quad$ APE-X-DQN Correction factor

$\beta_{r} \quad$ Effective rudder angle

$\boldsymbol{F}_{\boldsymbol{h}} \quad$ Hydrodynamic non-potential forces

$\boldsymbol{F}_{\boldsymbol{M}} \quad$ Fenders and mooring line forces

$\boldsymbol{F}_{\boldsymbol{p}} \quad$ Propulsion forces

$\boldsymbol{F}_{\text {tug }}$ Tugboat forces

$\boldsymbol{F}_{\boldsymbol{w} \boldsymbol{v}} \quad$ Wave forces

$\boldsymbol{F}_{\boldsymbol{w}} \quad$ Wind forces

$\delta(e)$ Temporal Difference Error for experience $e$

$\epsilon \quad$ Probability factor of taking random actions in $\epsilon$-greedy policies

$\gamma \quad$ Discount factor in a MDP

$\lambda \quad$ Advantage estimation limiting factor

$\pi(s) \quad$ Policy function that maps states to actions

$\rho \quad$ Water density

$\rho_{C L I P}$ PPO Clipping factor

$\theta \quad$ Policy network parameters

$A^{\pi}(s, a)$ Difference of expected accumulated reward of certain action

$A_{r} \quad$ Rudder area

$a_{t} \quad$ Action taken in a MDP for a given timestep 
$B \quad$ Training batch size of APE-X-DQN algorithm

$b \quad$ APE-X-DQN actor local buffer size

$B_{P P O}$ Training batch size for PPO iteration

c Context variable

$C_{L} \quad$ Dimensionless rudder lift coefficient

$D \quad$ APE-X-DQN Replay Buffer

$e \quad$ Experience set $\left\langle s, a, s^{\prime}, r, c\right.$, end_flag $>$

$F_{L} \quad$ Lift force on rudder

$F_{\text {ext }} \quad$ Sum of external forces

$I_{z} \quad$ Yaw moment of inertia of vessel

$k_{p r} \quad$ APE-X-DQN Prioritization constant

$L_{o a} \quad$ Vessel Length

$l_{r} \quad$ Neural network learning rate

$L_{\text {segment }}$ Desired segment length defined between two way-points

$M \quad$ Vessel Mass

$M_{11} \quad$ Vessel added mass in surge direction

$M_{22} \quad$ Vessel added mass in sway direction

$M_{26}$ Vessel coupled sway-yaw added moment of inertia

$M_{66} \quad$ Vessel yaw added moment of inertia

$N_{\exp }$ Minimum PPO experiences per training iteration

$N_{\text {ext }} \quad$ Yaw moment of external forces in vessel

$N_{\text {steps-per-iter }}$ Steps collected per training iteration

$p_{e} \quad$ APE-X-DQN Priority index for experience $e$

$Q^{\pi}(s, a)$ Expected accumulated reward after taking action $a$ at state $s$ for policy $\pi$ 
$r \quad$ Vessel yaw angular velocity

$r_{t} \quad$ Reward received in a given timestep

$s_{t} \quad$ State observed in a MDP for a given timestep

$T_{\theta} \quad$ APE-X-DQN target network update rate

$T_{\text {actor }}$ APE-X-DQN actor policy update rate

$u \quad$ Surge velocity in vessel coordinate system

$v \quad$ Sway velocity in vessel coordinate system

$V^{\pi}(s)$ Expected accumulated reward from state $s$ for policy $\pi$

$V_{r} \quad$ Rudder-fluid relative velocity

$w_{e} \quad$ APE-X-DQN Importance sampling factor

$W_{i} \quad$ Neural network weight for connection i

$X_{\text {ext }} \quad \mathrm{X}$-component of external forces in vessel local coordinates

$x_{G} \quad$ Gravity center location in $\mathrm{x}$ axis of vessel local coordinate system

$Y_{\text {ext }} \quad$ Y-component of external forces in vessel local coordinates

$\Delta T_{\text {eval }}$ Simulator numerical integration step at policy evaluation

$\Delta T_{\text {train }}$ Simulator numerical integration step during training

$\operatorname{cog}$ Course over ground 


\section{CONTENTS}

$\begin{array}{lll}1 & \text { Introduction } & 17\end{array}$

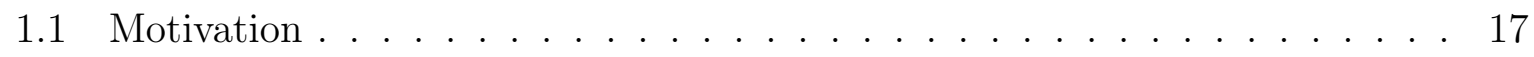

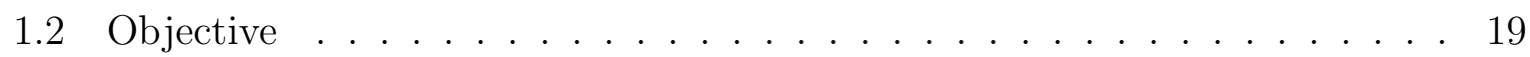

1.3 Text Structure . . . . . . . . . . . . . . . . . . . . . . 19

2 Background $\quad 21$

2.1 Vessel Dynamics . . . . . . . . . . . . . . . . . . . 21

2.2 Reinforcement Learning . . . . . . . . . . . . . . . . . . . . 24

3 Literature Review 33

4 Proposal $\quad 43$

4.1 State variables . . . . . . . . . . . . . . . . . . 44

4.2 Task Segmentation and Context Variable . . . . . . . . . . . . . . 46

4.3 Symmetry . . . . . . . . . . . . . . . . . . . . 49

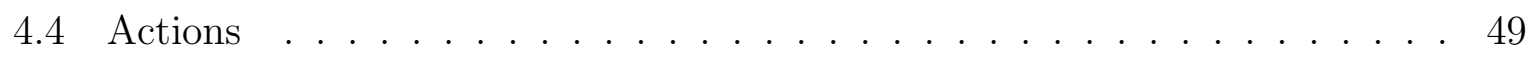

4.5 Reward ........................... 50

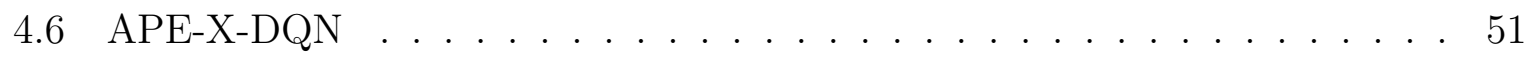

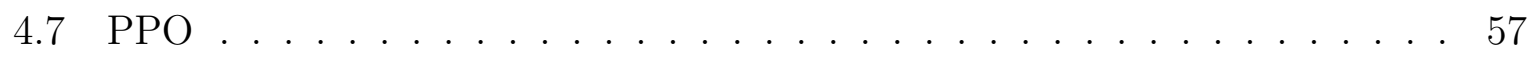

$\begin{array}{llr}5 & \text { Results } & 60\end{array}$

5.1 Training Settings . . . . . . . . . . . . . . . 6 60

5.2 Vessel Model and Training Scenario . . . . . . . . . . . . . . . 62

5.3 Generic Policy Training . . . . . . . . . . . . . . . . . . . 62

5.4 Evaluation in Porto Sudeste Channel . . . . . . . . . . . . . . 66 
5.5 Evaluation in Suape Channel . . . . . . . . . . . . . . . . . . . 74

5.6 Training in Porto Sudeste Channel . . . . . . . . . . . . . . . . . . . . 81

6 Conclusion and Next Steps $\quad 84$

$\begin{array}{lr}\text { Appendices } & 86\end{array}$

$\begin{array}{lr}\text { A Neural Networks } & 87\end{array}$

$\begin{array}{ll}\text { References } & 91\end{array}$ 


\section{INTRODUCTION}

\subsection{Motivation}

Even though automated systems are well established for navigation in open sea, when it comes to ports, bays and rivers - the so-called restricted waters - navigation is a very complex topic. Interference of margins and bottom in vessel dynamics is still a research field. Furthermore, navigation in restricted waters still relies on the knowledge of port authority regarding port and local weather conditions. A typical port entrance begins with the local pilot boarding. The pilot then emits verbal commands to helmsman for steering and propulsion. The vessel navigates through a channel delimited by buoys until a point where tugboats are attached to it through lines. The pilot gives commands to tugboats operators via radio until the vessel is towed to its final berth position.

Even with initiatives beginning to deliver prototypes of autonomous water vehicles (MUNIN, 2016) (LAURINEN, 2016) (KOOIJ; COLLING; BENSON, 2018), those still rely on adapted infrastructure and propulsion systems which differ from traditional ones. Autonomous port navigation still remains as an open topic.

Traditionally, most of the maritime navigation knowledge was obtained in real life situations (SPEIGHT R.; STRANNIGAN, 2015). Technology available around 40 years ago was limited and training offered to personnel on land regarded only standard rules and bridge procedures.

Along with the growth of vessel traffic (LLOYD J.; RODRIGUES, 2012), several incidents such as groundings and collisions started increasing. It has led to a real demand for skills improvement of mariners prior to boarding and then Ship Maneuvering Simulators (SMS) began to be developed.

SMS combine mathematical models which represent the behavior of water vehicles subjected to external disturbances. In Real-Time Simulations (RTS), several parameters can be set in order to train mariners for every possible situation they might encounter 
in real navigation. Over the last years, such simulators also started being applied to feasibility analysis of port projects.

RTS can be complex and expensive: They require physical infra-structure that emulates cabin controls and immersive displays; High personnel number is involved, including the presence of pilots and port authorities; It takes at least the amount of time required for the equivalent real operation. For these reasons, it is desirable to anticipate results in a efficient manner. Fast-Time Simulations (FTS) aim to attend that demand by providing vessel trajectories much faster without the need of professional pilots.

In FTS modality, mathematical models used in simulators are combined with planning and control algorithms, obtaining vessel trajectories through numerical integration. Dynamic obstacles are not considered and environmental conditions are assumed to remain constant in time. That serves many purposes, such as vessel model characterization in standard maneuvering tests, development of maneuvering plans in briefing sessions, among others (BENEDICT et al., 2017).

In case of port projects, FTS attempt to provide insightful information that can optimize RTS trials and also anticipate possible bottlenecks. The trajectories are obtained for vessels in new port scenarios or even unprecedented vessel models in existing ports.

There are several techniques that can be employed in FTS, but they usually comprise a higher level path planning stage, and a lower-level control tuning stage. In port channel scenarios, for instance, defining way-points can be trivial. However, current and wind forces can act transversely to the desired trajectory, causing collisions with margins. So as to overcome environmental effects, methods can account those in the way-point generation process, as in (ZACCONE; MARTELLI, 2018), or let lower-level control algorithm handle it. Once the desired path is determined, a control algorithm must be tuned, considering vessel model and the desired response, specially in curves. Environmental forces can be compensated with small drift angle by adjusting the yaw set-point (FOSSEN, 2011).

In some critical conditions, way-points and control parameters must be exhaustively re-adjusted in order to obtain satisfactory trajectories with no collision. This process can be very inefficient and labor costly. Moreover, the results obtained do not necessarily reflect the constraints imposed by real-world piloting. They may either exceed the pilot's acceptance criteria regarding safety distances or may demand an unfeasible number of engine and rudder commands.

The considerable research gaps in automatic navigation in ports and the issues involved in FTS trajectories present a great opportunity of exploring the application of 
machine learning to maritime domains. Besides that, the simulation infrastructure suits the intensive computational requirements of machine learning techniques and overcomes the slow and expensive nature of experiments with real vessel prototypes.

The fact that most scenarios adopted in FTS are unprecedented requires machine learning techniques that do not rely on previous navigation data. That naturally leads to Reinforcement Learning techniques, as they do not require explicit instructions from experts.

\subsection{Objective}

The overall objective of this work is to automatically generate vessel trajectories in port channels subjected to environmental conditions using Reinforcement Learning and Fast-Time Simulations. The scope of this work was bound to FTS analysis applications only. Generating a complete framework for navigating a real vessel autonomously would require a more complex study and several factors would need to be taken into account, as issued in (Van Den Boogaard et al., 2016) and (PERERA, 2018).

As means of simplification, simulations consider only navigation that begins at channel entrance and ends in the area that precedes tugboat attachment and towing process.

In order to provide trajectories that could be easily approximated in real-world piloting, the solution must consider the controllability limitations and safety criteria present in human pilot commanding. It must also improve FTS automation and exempt human efforts employed in the current workflow. Hence, the following prerequisites are defined:

- The commands obtained for control must be given in discrete intervals and also with predefined values.

- The distance from vessel center to margins must be higher than half of the vessel breadth.

- Only data available in the simulator must be consumed, dismissing prior route planning and control tuning.

\subsection{Text Structure}

In Chapter 2, some key concepts are introduced for the comprehension of the work. Beginning with vessel basic elements and dynamics. Then, machine learning concepts, 
such as neural networks, and finally Reinforcement Learning fundamentals.

Chapter 3 presents a literature review, where other works employing Reinforcement Learning in maritime navigation are listed and their commonalities are described.

In Chapter 4, the author elaborates a model for port channel navigation in conformity with Reinforcement Learning and justifies the simplifications and the inclusion of some elements. The algorithms adopted are also described.

In Chapter 5, the experiments in the simulator are described in terms of scenario and model specifications; the results obtained are shown and analyzed.

The author finally concludes in Chapter 6, presents the next steps for the work developed and some long-term directions of the research. 


\section{BACKGROUND}

This chapter gives an overview of concepts of physical nature related to the maritime domain simulated in the experiments and also reviews fundamentals upon which the techniques adopted in this work were built. In Section 2.1, basic description of vessels movement and propulsion are provided as well as dynamic equations. Section 2.2 presents the basics of Reinforcement Learning framework, shortly describes notable algorithms and subtopics related to it.

\subsection{Vessel Dynamics}

Mechanical propulsion in vessels generally consist of a motor or engine turning a propeller, or less frequently, an impeller or wave propulsion fins. Steam engines were first used for this purpose, but have mostly been replaced by two-stroke or four-stroke diesel engines, outboard motors, and gas turbine engines on faster vessels. Nuclear reactors producing steam are used to propel warships and icebreakers, and there have been attempts to utilize them to power commercial vessels

In addition to traditional fixed and controllable pitch propellers there are many specialized variations, such as counter-rotating and nozzle-style propellers. Most vessels have a single propeller, but some large vessels may have up to four propellers supplemented with transverse thrusters for maneuvering at ports. The propeller is connected to the main engine via a propeller shaft and, in case of medium- and high-speed engines, a reduction gearbox. Some modern vessels have a diesel-electric power train in which the propeller is turned by an electric motor powered by the vessel's generators.

In most vessel designs, a steering system becomes necessary. The most common is a rudder (Figure 1), a submerged plane located at the rear of the hull. Rudders are rotated to generate a lateral force which turns the boat. Rudders can be rotated by a tiller, manual wheels, or electro-hydraulic systems. Autopilot systems combine mechanical rudders with navigation systems. The rudder operates by redirecting the water flow generated by the 
propeller, thus imparting a turning or yawing motion to the vessel.

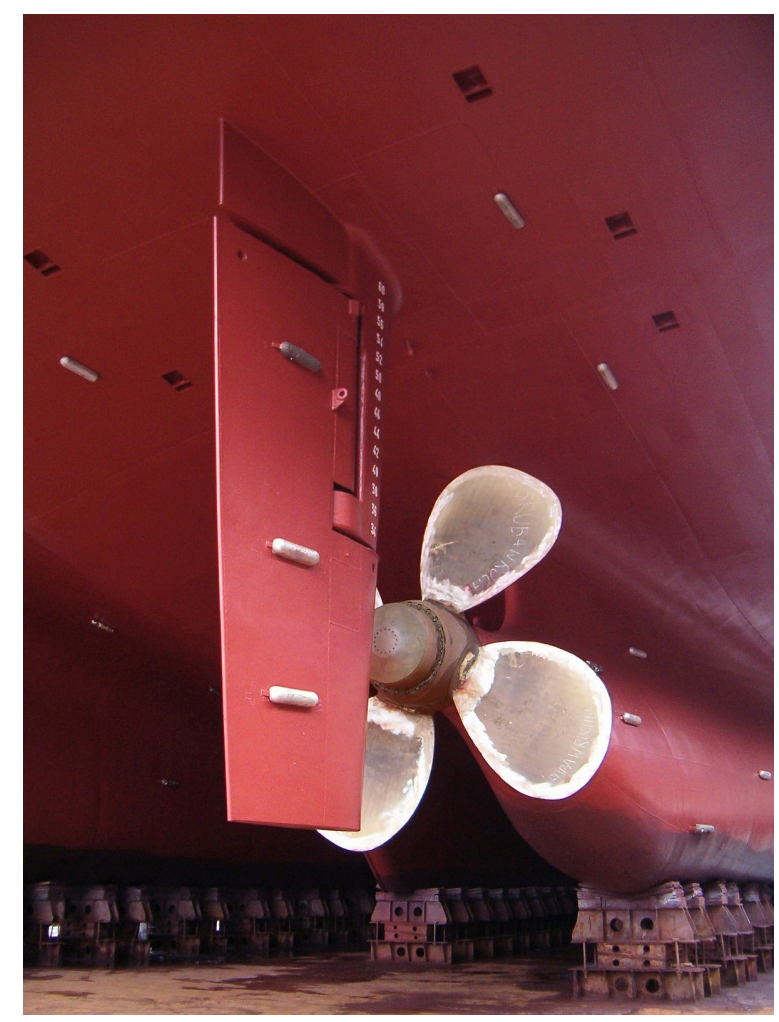

Figure 1: Rudder and propeller of a vessel

The mathematical models represent the motion of a floating vessel at low speed in 6 DOF (degrees of freedom), subjected to the external forces due to the environmental and tugboats and to the control forces provided by the thrusters, propeller and rudder. The 6 DOF vessel dynamics differential equations are solved using explicit 4rd order RungeKutta integration method, considering the interaction with the fluid and the external forces acting on the hull. However, as a matter of simplicity, this section will only present the equations of motion for the horizontal plane. Two different coordinate systems to derive the vessel equations of motions are adopted, as shown in Figure 2. The system $O X Y Z$ is earth-fixed (inertial system) and the system $O x y z$ vessel-fixed, with the origin on central point of the keel midship section. The center of gravity $\mathrm{G}$ is at the distance $x_{G}$ ahead from the point o, ox is the longitudinal axis of the vessel directed to the bow, and oy is the transversal axis, pointing to port. The heading of the vessel $\psi$ defines the angle between the $o x$ and $O X$ axes.

Following (FOSSEN, 2011), the horizontal 3 DOF equations of motion referred to the body-fixed Oxyz coordinate system, considering symmetry with respect to the axis ox, are given by:

$$
\left(M+M_{11}\right) \dot{u}-\left(M+M_{22}\right) v r-\left(M x_{G}+M_{26}\right) r^{2}=X_{e x t},
$$




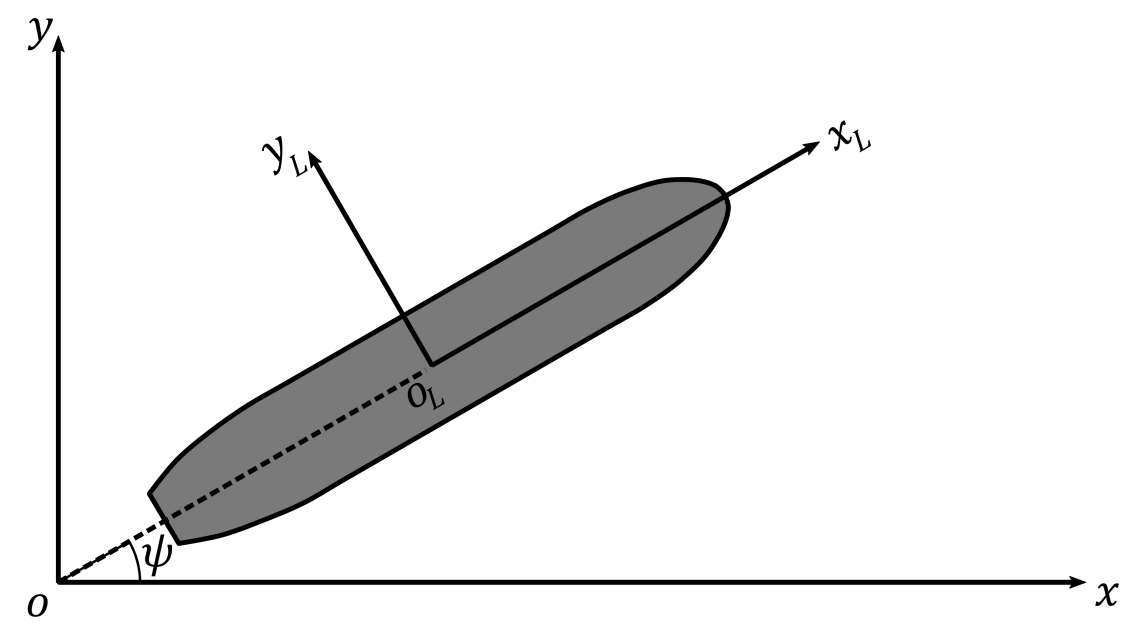

Figure 2: Vessel coordinate system

$$
\left(M+M_{22}\right) \dot{v}+\left(M x_{G}+M_{26}\right) \dot{r}+\left(M+M_{11}\right) u r=Y_{e x t}
$$

and

$$
\left(I_{z}+M_{66}\right) \dot{r}+\left(M x_{G}+M_{26}\right)(\dot{v}+u r)+\left(M_{22}+M_{11}\right) u v=N_{e x t},
$$

where $M$ is the vessel mass, $I_{z}$ is the yaw moment of inertia of the vessel, $u$ and $v$ are the surge and sway velocities respectively and $r$ is the yaw angular velocity. The terms $M_{11}$ and $M_{22}$ are the vessel added masses in the surge and sway directions, $M_{66}$ is the vessel added moment of inertia and $M_{26}$ is coupled sway-yaw added inertia. $X_{\text {ext }}, Y_{\text {ext }}$ represent the $\mathrm{X}$ and $\mathrm{Y}$ components of external forces and $N_{\text {ext }}$. The external forces can be expressed in terms of different factors, as in:

$$
\boldsymbol{F}_{\text {ext }}=\boldsymbol{F}_{h}+\boldsymbol{F}_{\boldsymbol{w}}+\boldsymbol{F}_{\boldsymbol{w} v}+\boldsymbol{F}_{\boldsymbol{p}}+\boldsymbol{F}_{\text {tug }}+\boldsymbol{F}_{\boldsymbol{M}}
$$

where $\boldsymbol{F}_{\boldsymbol{h}}$ represents the hydrodynamic non-potential forces, including the current and maneuvering forces, $\boldsymbol{F}_{\boldsymbol{w}}, \boldsymbol{F}_{\boldsymbol{w} \boldsymbol{v}}$ represent the wind and wave forces, respectively, $\boldsymbol{F}_{\boldsymbol{p}}$ represents the thrusters, propeller and rudder forces, $\boldsymbol{F}_{\boldsymbol{t u g}}$ represents the external action of the tug boats, either in contact with the hull or connected by a cable and $\boldsymbol{F}_{\boldsymbol{M}}$ represents the forces due to mooring lines, fenders or anchor lines. In the present work, the vessel navigates along the channel without the help of any tugboats or attached to any lines, being only controlled by its own propeller and rudder under environmental forces. Therefore, the terms $\boldsymbol{X}_{t u \boldsymbol{g}}$ and $\boldsymbol{X}_{\boldsymbol{M}}$ are null in the equation 2.4 .

A physical-based model is used for the calculation of the rudder forces (MOLLAND; TURNOCK, 2007). The forces are illustrated in Figure 3 and rudder lift force $F_{L}$ is given 
by:

$$
F_{L}\left(\beta_{r}\right)=0.5 \rho A_{r} C_{L}\left(\beta_{r}\right) V_{r}^{2}
$$

where $\rho$ is the water density, $A_{r}$ is the rudder area, $C_{L}$ is a rudder dependent dimensionless coefficient, $\beta_{r}$ the effective rudder angle and $V_{r}$ the relative velocity of the fluid onto the rudder.

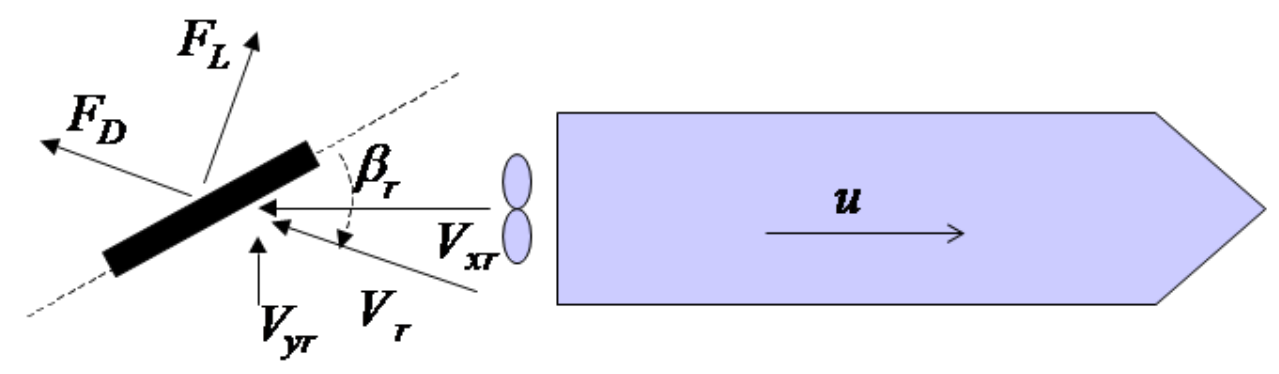

Figure 3: Forces on the rudder

It can be noted that the rudder forces are dependent on the speed of the water on the rudder. This water jet is mainly generated by the propeller rotation, indicating that the vessel will be more controllable (maneuverable) if the propeller is operating. With engine stopped, the water flow on the rudder and the rudder forces will be reduced, making the vessel very difficult to be controlled.

In port navigation, the vessel is controlled only by a main propeller and a rudder and can not directly be commanded to follow arbitrary trajectories in configuration space. Under this regime it is usually referred as under-actuated vessel.

\section{$2.2 \quad$ Reinforcement Learning}

Artificial Intelligence is a field of computer science whose definition may vary among authors. Most of the literature define it as the science that studies systems which fall in one the four categories (RUSSELL; NORVIG, 2009): Those that think like humans, acts like humans, think rationally or that act rationally. Machine Learning is the subset of Artificial Intelligence dealing with computational algorithms which build a mathematical model of data in order to make predictions or decisions without being explicitly programmed to perform a certain task (BISHOP, 2013). There are three basic learning paradigms: Supervised, Unsupervised and Reinforcement Learning.

In Supervised Learning paradigm, learning process occurs from a set of labeled examples provided by external supervisor. Each example is a situation described by features together with the label of the correct action the system should take in that situation. 
That is the case of a classification task, where the agent must identify a category to the input. The goal of this paradigm is to generalize its responses so that it acts correctly in situations not present in the training set.

Unsupervised learning algorithms take a set of data that contains only inputs, and find structure in the data, like grouping or clustering of data points. The algorithms therefore learn from test data that has not been labeled, classified or categorized. Instead of responding to feedback, unsupervised learning algorithms identify commonalities in the data and react based on the presence or absence of such commonalities in each new piece of data.

Reinforcement Learning (RL) (SUTTON; BARTO, 2018) is the Machine Learning subset which studies a certain class of problems and respective solutions, where a decision for an action must be made based on the observations of a given situation and reward values. Figure 4 depicts the basic scheme of RL problems.

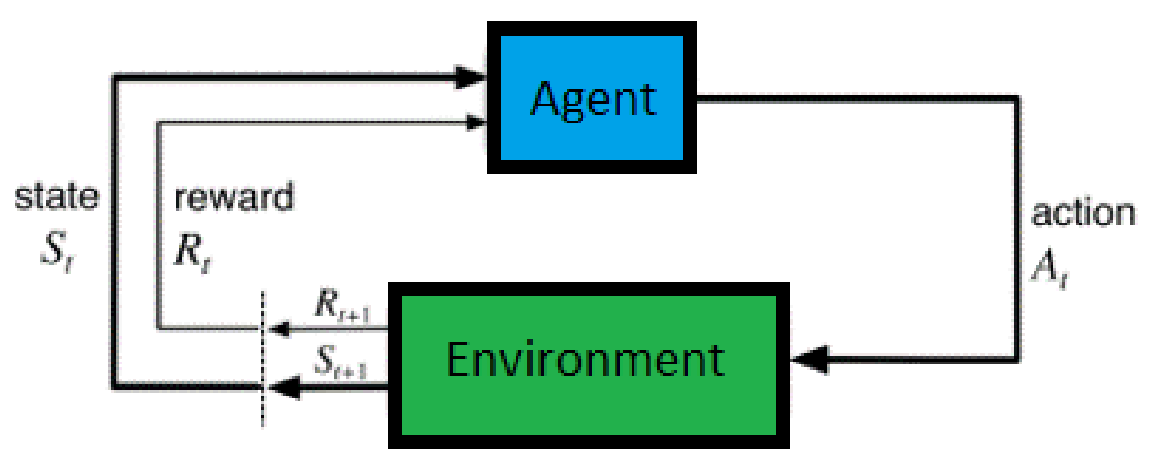

Figure 4: RL basic scheme

Differently from Supervised Learning, RL does not require labeled examples. Obtaining examples of desired behavior that are representative and can be extrapolated to situations in which the agent has to act are often unfeasible in interactive problems. In this sense, one RL advantage is that the agent learns from its own experience.

Beyond the agent and the environment, other important elements of RL system are: a policy, a reward signal and, optionally, a model of the environment.

RL algorithms have primarily been developed to identify optimal decisions in a formal class of tasks known as Markov Decision Processes (MDP). They are a class of decisionproblem stripped down enough to be amenable to fairly straightforward mathematical analysis, while still covering a broad range of tasks and preserving a number of the elements of nontrivial real world decisions. The core elements in a MDP are: 
- A set of states $S$ (ex: in chess, a state is the board configuration)

- A set of possible actions $A$ (In chess, all the move that could be possible in every configuration possible, eg: e4-e5)

- The conditional distribution $P: S \times A \times S \mapsto[0,1]$ of next states given a current state and an action. In a deterministic environment like chess, transitioning from state $s$ with action $a$, there is only one state $s^{\prime}$ with probability 1 , and all the others have probability 0 . Nevertheless, in a stochastic environment involving randomness (ex: a coin toss), the distribution is not as simple.

- Reward function $R: S \times A \times S \mapsto \mathbb{R}$ for state transitions

Formally, an environment is defined as a MDP. It has the so called discrete dynamics (as opposed to continuous time dynamics): The world's state takes on a new value from $S$ at each timestep $t$ and also chooses an action $a$ from $A$, finally receiving a reward signal $r$, which measures the utility received on that timestep.

The actions are important because they influence the evolution of the state, and hence the obtained rewards. The most important simplifying assumption of the MDP is the Markov property, which assumes that the state transition probability depends only upon the current state and action. Conditional on these, the new state is independent of all earlier states and actions. Rewards also obey the Markov property, since they depend only on the current state transition and, conditional on this, are independent of any earlier history.

By constraining the relationships between events across time, the Markov conditional independence property simplifies analysis, learning, decision making and is key to RL algorithms. The behavior of the agent in an MDP can be defined as a probability distribution $\pi: S \times A \rightarrow[0,1]$ called a policy, which given $s \in S$ and $a \in A$, represents the probability of selecting $a$ as next action from $s$. An agent that uses this probability distribution to select its next action when in a given state is said to be following the policy. In case of deterministic environments, the policy directly maps a state to a given action.

In 1950s a method called Dynamic Programming (DP) (BELLMANN, 1957) was developed in order to solve this optimization problem when there is a complete knowledge on transitions distributions. DP breaks a multi-period planning problem into simpler steps at different points in time. Therefore, it requires keeping track of how the decision situation is evolving over time. The information about the current situation that is 
needed to make a correct decision is called the "state" and the evaluation of how good it is for an agent following a policy $\pi$ to be in a given state is expressed in terms of the expected discounted sum of future rewards collected by an agent starting from a state while following $\pi$.

The optimality condition occurs when the policy obtained leads to the highest discounted reward sum, or also referred as maximum return. In that case, the value $V^{*}$ of a state associated to an optimal policy must be the expected return of the best action that the agent can take in that state. The Bellman Optimality Equation can be written in terms of value of the subsequent state when following the optimal policy, as:

$$
\begin{aligned}
V^{*}(s) & =\max _{a} Q^{*}(s, a) \\
& =\max _{a} E_{\pi^{*}}\left[R_{t} \mid s_{t}=s, a_{t}=a\right] \\
& =\max _{a} E_{\pi^{*}}\left[\sum_{k=0}^{\infty} \gamma^{k} r_{t+k+1} \mid s_{t}=s, a_{t}=a\right] \\
& =\max _{a} E_{\pi^{*}}\left[r_{t+1}+\gamma \sum_{k=0}^{\infty} \gamma^{k} r_{t+k+2} \mid s_{t}=s, a_{t}=a\right] \\
& =\max _{a} E_{\pi^{*}}\left[r_{t+1}+\gamma V^{*}\left(s_{t+1}\right) \mid s_{t}=s, a_{t}=a\right] \\
& =\max _{a} \sum_{s^{\prime} \in S} P\left(s, a, s^{\prime}\right)\left[r(s, a)+\gamma V^{*}\left(s^{\prime}\right)\right],
\end{aligned}
$$

where $Q^{*}(s, a)$ is the expected return for optimal policy if action $a$ is taken at state $s$ and $P\left(s, a, s^{\prime}\right)$ is the transition probability function in case of stochastic environments.

For practical purposes, it is desirable to have a value of expected future return written in terms of a given action $a$ taken in a given state $s$. This is known as the action value or also "Q" value. The Optimality equation adapted for $\mathrm{Q}$ is described as:

$$
\begin{aligned}
Q^{*}(s, a) & =E\left[r_{t+1}+\gamma \max _{a^{\prime}} Q^{*}\left(s_{t+1}, a^{\prime}\right) \| s_{t}=s, a_{t}=a\right] \\
& =\sum_{s^{\prime}} P\left(s, a, s^{\prime}\right)\left[r(s, a)+\gamma \max _{a^{\prime}} Q^{*}\left(s^{\prime}, a^{\prime}\right)\right] .
\end{aligned}
$$

Non-episodic tasks are considered infinite in time and occur, for example, in control problems, where it is desired to keep at a certain set-point in the infinite horizon. In order to avoid infinite values for accumulated reward, the discount factor $\gamma$ is introduced. The 
closer the factor gets to 1 , the more it takes future rewards into account. The closer to 0 , more short-sighted the decisions become.

The two approaches used in DP are Value Iteration and Policy Iteration. Policy Iteration works by iteratively computing the value function associated to the current policy, and then improving that policy by making it act greedily with respect to the value function, such as:

$$
\pi^{\prime}(s)=\underset{a \in A}{\arg \max } Q^{\pi}(s, a)
$$

This continuous improvement is applied until the improved policy satisfies the Bellman Optimality equation. Since the algorithm gives no assurances on the number of updates required for convergence, some stopping conditions are usually introduced to end the process when the new value function does not change substantially after the update or a certain threshold number of iterations has been reached.

Based on a similar idea, Value Iteration approach starts from an arbitrary value function, and then applies a contraction operator which iterates over sequentially better value functions without actually computing the associated greedy policy. The contraction operator which ensures convergence is the Bellman Optimality Backup, given as:

$$
V_{k+1}(s) \leftarrow \max _{a} \sum_{s^{\prime}} P\left(s, a, s^{\prime}\right)\left[r(s, a)+\gamma V_{k}\left(s^{\prime}\right)\right]
$$

where $k$ represents the iteration step. As with Policy Iteration, convergence is ensured without guarantees on the number of steps, and therefore it is usual to terminate the iteration according to some stopping condition.

As the model is not known, it is not possible to solve the system of equations associated to the value functions and DP can no longer be applied. It is import to distinguish analytic models, such as dynamic equations, from generative models, which represent the probability distribution or the exact sequence of state transitions. This last one is required for DP algorithms and, although analytic models might even be available in some situations, obtaining generative models might be very costly. Reinforcement learning, then, employs other methods to find the value function without relying on prior generative models. The family of RL methods which focus on estimating the value function for states or action-state pairs are known as Value-based methods.

RL algorithms are classified as online if action values are updated during interaction with the environment. Batch RL algorithms (LANGE; GABEL; RIEDMILLER, 2012) are offline and do not attempt to learn as the agent interacts with the environment. They 
are designed to be more sample efficient(RIEDMILLER, 2005) (ANTOS; SZEPESVÁRI; MUNOS, 2008), as they can store a number of interactions with the environment and use the data multiple times for learning. Additionally, such methods allow a clear separation of the learning mechanism from the exploration mechanism (which much decide whether to attempt to gather more data about the environment or exploit the current best policy).

Temporal Difference (TD) methods (SZEPESVÁRI, 2010) estimate value functions by using in part an already computed estimate. This approach is called bootstrapping. Instead of the average of all rewards following the visit, a single or few time steps are considered.

For TD methods, random exploration of the environment will converge to the optimal policy, but it is only guaranteed after an almost infinite time, if every sequence of possible transitions is visited at least once. Considering how many states and branching exist, it is impossible to reach the optimal policy for a typical problem and most sub-optimal policies are adopted. The convergence to at least a reasonably good policy can be achieved through different ways, and one of them is $\epsilon$-greedy exploration. $\epsilon$-greedy is a policy that chooses an action at random with odd $\epsilon$ or the best action as deemed by our current policy with odd $(1-\epsilon)$. Usually $\epsilon$ is annealed over time to privilege exploitation of the policy learned over random actions after enough exploration. This is a trade-off between exploration and exploitation.

At each new information, our actual Q functions get more accurate about the present policy and the exploration is focused on better paths. The policy based on our new $Q$ function gets better (since Q is more accurate) and the $\epsilon$-greedy exploration reach better paths. Focused on those better paths, Q function explore even more the better parts and has to update its returns according to the new policy. This is an iterative cycle that enables convergence to the optimal policy called policy iteration (Illustrated in Figure 5). Unfortunately, the convergence can take infinite time and is not even guaranteed when $\mathrm{Q}$ is approximated by neural networks. Nevertheless, impressive results can make up for the lack of formal convergence proofs.

A RL algorithm is classified as on-policy if it attempts to evaluate and improve the same policy that they use to make decisions, and off-policy if it has no relations between the estimated policy and the policy used to collect experience.

Q-Learning (WATKINS; DAYAN, 1992) is an online, off-policy algorithm largely used in RL. The main idea of the algorithm is that an agent interacts with its environment as follows: in each time step, the agent observes the state $s_{t}$ and decides the action $a_{t}$ to be 


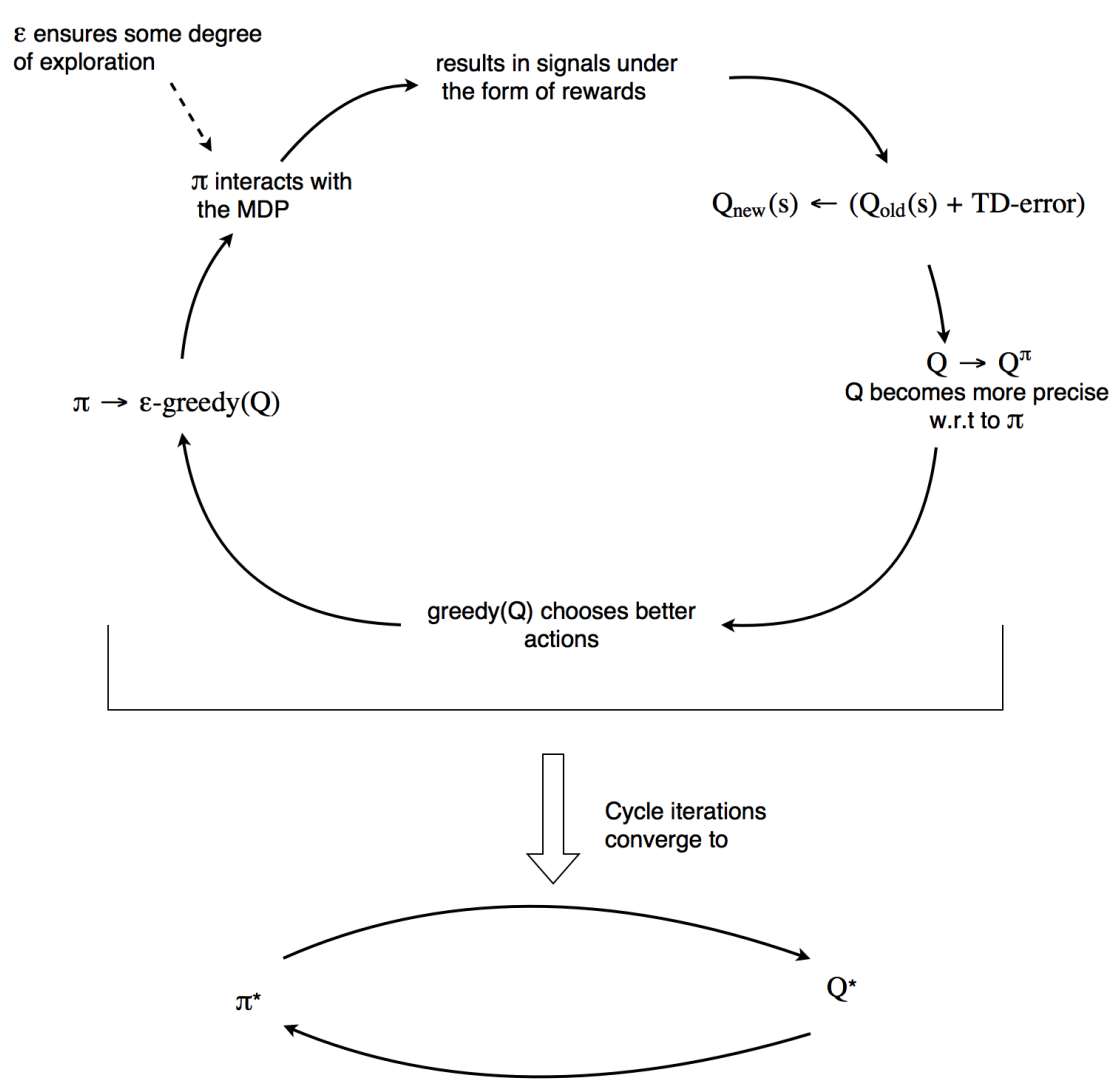

Figure 5: Policy Iteration

taken and performs it; the environment then transitions to the next state $s_{t+1}$ and the agent receives a reward $r_{t}$ as feedback. This cycle of interaction between the agent and the environment defines an experience, given by the tuple $\left\langle s_{t}, a_{t}, r_{t}, s_{t+1}\right\rangle$. This experience is used to iteratively estimate the state-action value function $Q$. The update rule of Q-Learning is

$$
Q\left(s_{t}, a_{t}\right) \leftarrow(1-\alpha) Q\left(s_{t}, a_{t}\right)+\alpha\left(r_{t}+\gamma \max _{a_{t+1}} Q_{t-1}\left(s_{t+1}, a_{t+1}\right)\right),
$$

where $\alpha$ is the learning rate $(0<\alpha \leq 1)$. The difference between the target value and the current value is commonly referred to as Temporal Difference (TD) Error. It can be proven that $Q$ values converge to the optimal policy in the limit if all states are sufficiently explored. For continuous state-spaces, Q-learning can be extended to its approximation form, using for example supervised learning techniques for getting an estimate of a $\mathrm{Q}$ function.

While Q-Learning and other TD methods are value-based algorithms because they estimate $\mathrm{Q}$ values for further action selection, there are also Policy Gradient (PG) algorithms. They act by optimizing estimators that directly output a continuous action 
or parameters for action probability distributions. In a problem with discrete actions, the policy estimates the probability of each action in the discrete set. In the continuous actions space, on the other hand, the policy approximates the variance and the mean of a distribution. The policy weights $\theta$ are learned according to an approximate gradient of a performance indicator $J(\theta)$, where $p_{\theta}$ is the probability of a given trajectory $\tau$ (or sequence of experiences) occur when following policy $\pi_{\theta}$ and $R(\tau)$ is the expected sum of rewards for such sequence, as in:

$$
J(\theta)=\int p_{\theta}(\tau) R(\tau) d \tau
$$

The gradient, after using the convenient property $\nabla p_{\theta}(\tau)=p_{\theta}(\tau) \nabla \log p_{\theta}(\tau)$, becomes an expected value, as given by:

$$
\nabla J(\theta)=\int p_{\theta}(\tau) \nabla \log p_{\theta}(\tau) R(\tau) d \tau=\mathbb{E}_{\tau(\tau)}\left[\nabla \log p_{\theta}(\tau) R(\tau)\right]
$$

and can, thus, be approximated with sampling of a sequence of $T$ state transitions, as in

$$
\nabla J(\theta) \approx \frac{1}{N} \sum_{i=1}^{N}\left(\sum_{t=1}^{T} \nabla \log \pi_{\theta}\left(a_{i, t} \mid s_{i, t}\right)\right)\left(\sum_{t=1}^{T} r\left(s_{i, t}, a_{i, t}\right)\right) .
$$

In order to eliminate the bias of the estimation, the value $V(s)$ is subtracted from the estimation of reward sum. Since it is not related to $\theta$, the gradient remains unaltered. The term obtained is known as Advantage Function, given by:

$$
A^{\pi}(s, a)=Q^{\pi}(s, a)-V^{\pi}(s)
$$

and represents how good the choice of a certain action is from a state when compared from the expected accumulated return from that state under a given policy.

They are applicable to a wider range of problems since they can produce stochastic policies and continuous actions. PG algorithms also have drawbacks: they have the tendency of converging to local optima and are more sample inefficient.

Deep RL combines RL with complex neural network (See Appendix A) architectures so as to approximate the action-value function. Deep Q Network (DQN), a Deep RL algorithm, gained notoriety when it was used to solve ATARI games (Mnih et al., 2015). A DQN variant was adopted in this work (Section 4.6).

One source of instability in training are the correlations in the sequence of observations 
and changes in data distribution due to policy change. DQN tackles this problem using a delayed copy of the neural network to estimate Q-values used in the TD error. It also adopts experience replay, where it stores transition data in a replay memory buffer and selects a batch of random transitions to fit the network at every learning step. The probability of choosing a transition can vary according to the problem an can even benefit experiences that do not occur very often.

There are also PG algorithms that use ANN estimators. One of them is Proximal Policy Optimization (PPO) (SCHULMAN et al., 2017) and it is also evaluated in the present work (Section 4.7), as well as in some of the works reviewed in Chapter 3. 


\section{LITERATURE REVIEW}

RL has demonstrated very satisfactory performance in many control applications, overcoming domain complexities such as nonlinear dynamics, varying set-points, long-term dynamic effects, influence of external variables and the primacy of precision (HAFNER; RIEDMILLER, 2011a). Some real-world physical applications gained notoriety, such as control of autonomous helicopter, where it was demonstrated the ability of acrobatic movements such as inverted position flight (NG et al., 2006). Even in domains such as robotics (KOBER; BAGNELL; PETERS, 2013), where usual RL applications deals with high-level decision making (KOBER; BAGNELL; PETERS, 2013), RL has recently accomplished dexterous tasks in a end-to-end fashion without any prior knowledge on the physical model, such as door opening with robotic hands (ZHU et al., 2019).

A search for articles applying RL specifically to maritime navigation was performed in Google Scholar platform. The keywords "Reinforcement Learning", "Vessels", "Ships" where combined in the search and all conference and journal articles in English language were considered. From that amount, only works dealing with RL specifically for controlling vessel or assigning a trajectory to it were reviewed. Applications that dealt with tugboats and super actuated maneuvering in the final berthing phase were disregarded since those tasks are out of the scope of this dissertation. Table 1 lists the works ordered by year and describes their task, as well as their formulations as RL problems, the algorithms employed and the training settings adopted.

Apart from publications originated within this dissertation, the only reviewed work which aimed to obtain trajectories in a restricted channel was also the oldest article found in literature: (STAMENKOVICH, 1992) trained an agent in broad channels with quite simple value approximation; The ship avoided collision, but navigated too close to margins in curves and presented oscillatory trajectory. Although this work should be acknowledged for its proposal, it is outdated and limited by the computational resources available back in its publication. Its dynamics simplification and its policy behavior do not attend navigation in straight channels with a variety of environment conditions. 
Some applications regarded path planning tasks in maritime navigation. Following definitions of (ZHOU et al., 2020), path planning have 3 stages: Route planning, Trajectory Planning and Motion Planning. Route planning considers the vessel as a particle point and does not take into account its dynamics. Considering a pre-defined start point and goal point, the question being answered here is "Where should the vessel go next?" regardless the vessels actions. (WANG et al., 2018), (ZHANG et al., 2019) and (WU et al., 2020) used RL in its discrete form to strictly define points of trajectory. After defining trajectory points, another stage for defining vessel motions would be required. Figure 6 portrays the route planning problem.

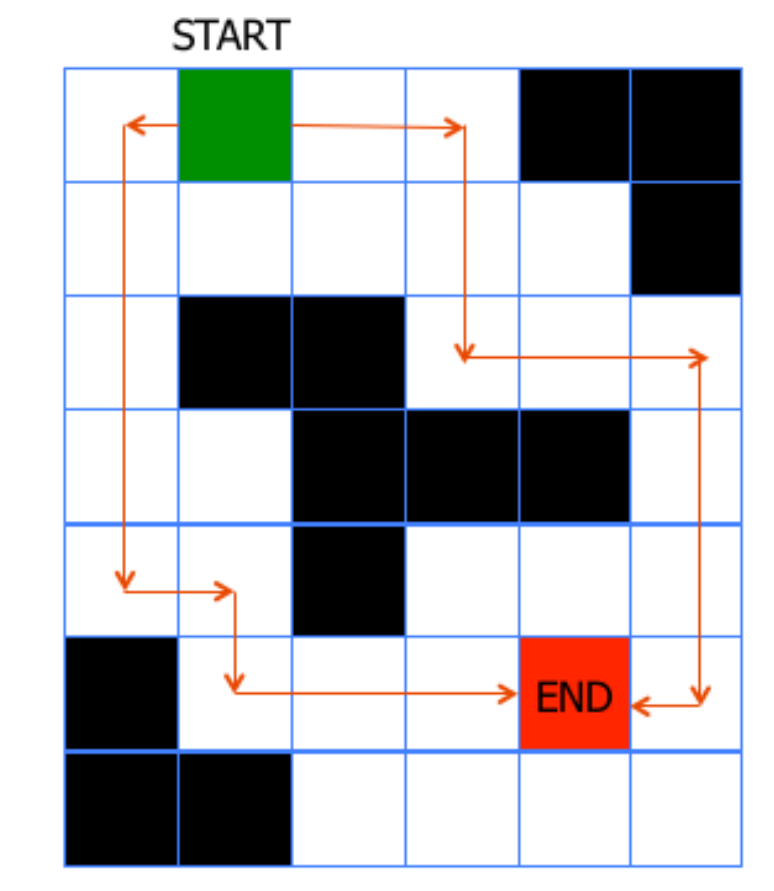

Figure 6: Route planning for areas discretized in grids

Trajectory planning include dynamics partially. Motion planning includes complete dynamics and aims to obtain the sequence of low-level actions in order to make the vessel reach its goal. Examples of RL applications that cover up to this final stage in scenarios with static obstacles are (RAK; SC, 2012), (TUYEN et al., 2017). Some of motion planning tasks ((NAKAYAMA et al., 2014), (CHENG; ZHANG, 2018), (ZHANG et al., 2019), (GUO et al., 2020)) also regard scenarios with multiple vessels, where trajectories must be generated avoiding collision among them.

When trajectories are already defined through way-points, the motion is often defined by path following algorithms in order to make vessels navigate as close as possible to segments formed. In order to make under-actuated vessels accomplish that, marine 
control systems often employ a technique called Line-of-Sight (LOS) (FOSSEN, 2011): As illustrated in Figure 7, LOS consists of defining a circle with fixed radius centered in the controlled vessel; The intersection between the circle and the path sets the instant course set-point to be pursued by the control algorithm.

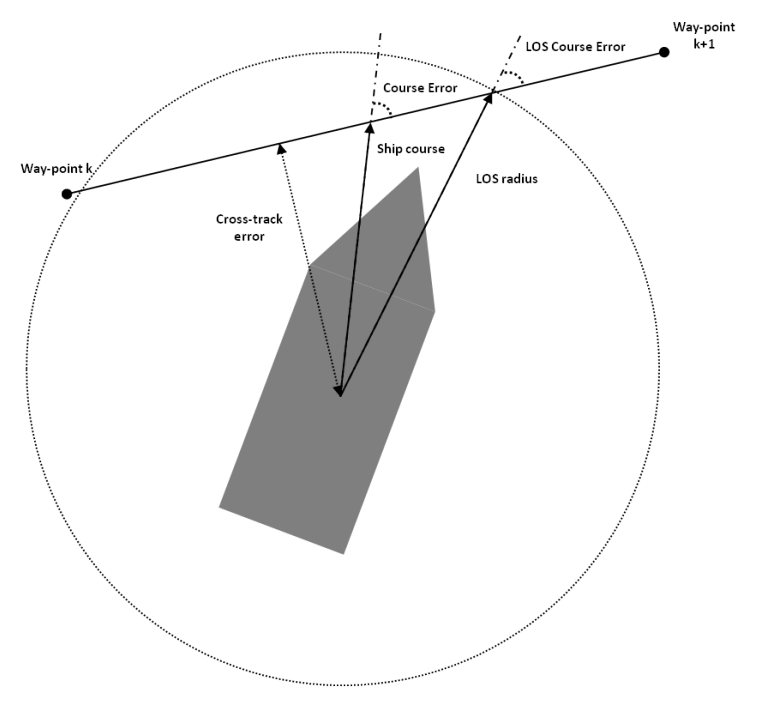

Figure 7: Line of Sight Technique and set-point error measurements

After state-of-art algorithms started combining RL with Deep Neural Networks in complex benchmark domains, some maritime path following applications using Deep RL emerged. In such applications, RL episodes are modelled with indefinite length and a higher dimension state space with information about error relative to desired path. Some of them adopted LOS strategy for setting error variables observed in state-state (ZHAO; ROH, 2019), others considered the current segment orientation in the state space (MARTINSEN; LEKKAS, 2018b) and even adopted curved paths (MARTINSEN; LEKKAS, 2018a). So as to tackle autonomous vessels issues, recent collision avoidance was combined with path following (ZHAO; ROH, 2019), (MEYER et al., 2020). Their state-space and reward representation are extended with information - eg.: distance - about other vessels or obstacles.

Two works were published as result of the elaboration of the present dissertation. Channel navigation was handled as a path-following problem. In (AMENDOLA et al., 2018), the port channel adopted in the experiments was straight and no environment conditions were considered, the state space adopted was simpler and an algorithm called Fitted Q Iteration was used. The work was improved in (AMENDOLA et al., 2019): Although curves were still not regarded, it addressed the complexity of the task by training different agents for wind, current and wave forces. Both state space and reward functions adopted were refined, but each training process occurred in a single scenario and provided 
successful trajectories with limited range of current and wind variations.

State space in path planning tasks carries information about the absolute position in a given scenario. The policy, in this case, is learned for that specific setup with very limited capacity of generalization. In path following tasks, state space uses information relative to closest segment to be followed and the policy is easily extended for path segments of any length.

In path following scenarios that include obstacles, training episodes vary the scenario disposition - in case of multiple vessels, different positions and orientations. It occurs as a strategy to obtain policies that are more general and respond reasonably to unexpected situations. That strategy was similarly adopted in the presence of ocean current: as they were not included in state space, (MARTINSEN; LEKKAS, 2018b) varied their value in order to obtain a policy robust against small current fluctuations.

Earlier motion planning applications showed that using RL in its tabular form for lowlevel control can be computationally expensive (LACKI, 2008), (RAK; SC, 2012). More recent works used neural networks for Q-value approximation (CHENG; ZHANG, 2018). Discrete actions also incorporated handcrafted rules in (NAKAYAMA et al., 2014), where action selection is limited when some choices lead the vessel towards situations with risk of collision. Most RL path following represented the rudder actions in continuous space and adopted Deep RL algorithms based on policy gradient (MARTINSEN; LEKKAS, 2018b), (MEYER et al., 2020). As exception, (AMENDOLA et al., 2018) and (AMENDOLA et al., 2019) adopted few discrete rudder commands as action space in order to incorporate human piloting limitations.

Reward signals employed in path planning were often defined as high values when goal is reached, large magnitude negative values for collision with obstacles and small magnitude negative values for every other state. Path following tasks used continuous functions that decrease when the vessels moves away from the path. The collision with vessels was also accounted in reward representations: (MEYER et al., 2020) added a punishment function that turned more negative as other vessels approached; Maritime navigation rules - e.g: Which course should a vessel take when another vessel approaches were embedded in reward function (ZHAO; ROH, 2019). A state-action pair was punished whenever a rule was not respected by the controlled vessel.

Although much of the representations recently proposed for path following tasks can be used as benchmark for the present work, they present some gaps for the purpose of the fast-time simulations. They did not include any information about environmental 
conditions in their state representation, which means the robustness of policy gets compromised under higher variations in direction and intensity of wind, waves or current. The constraints imposed by the channel margins are critical for policy learning - specially in curves - and they are not present in those works. Also, the recent Deep RL techniques employed continuous actions in continuous time, which do not correspond to real world-piloting. 


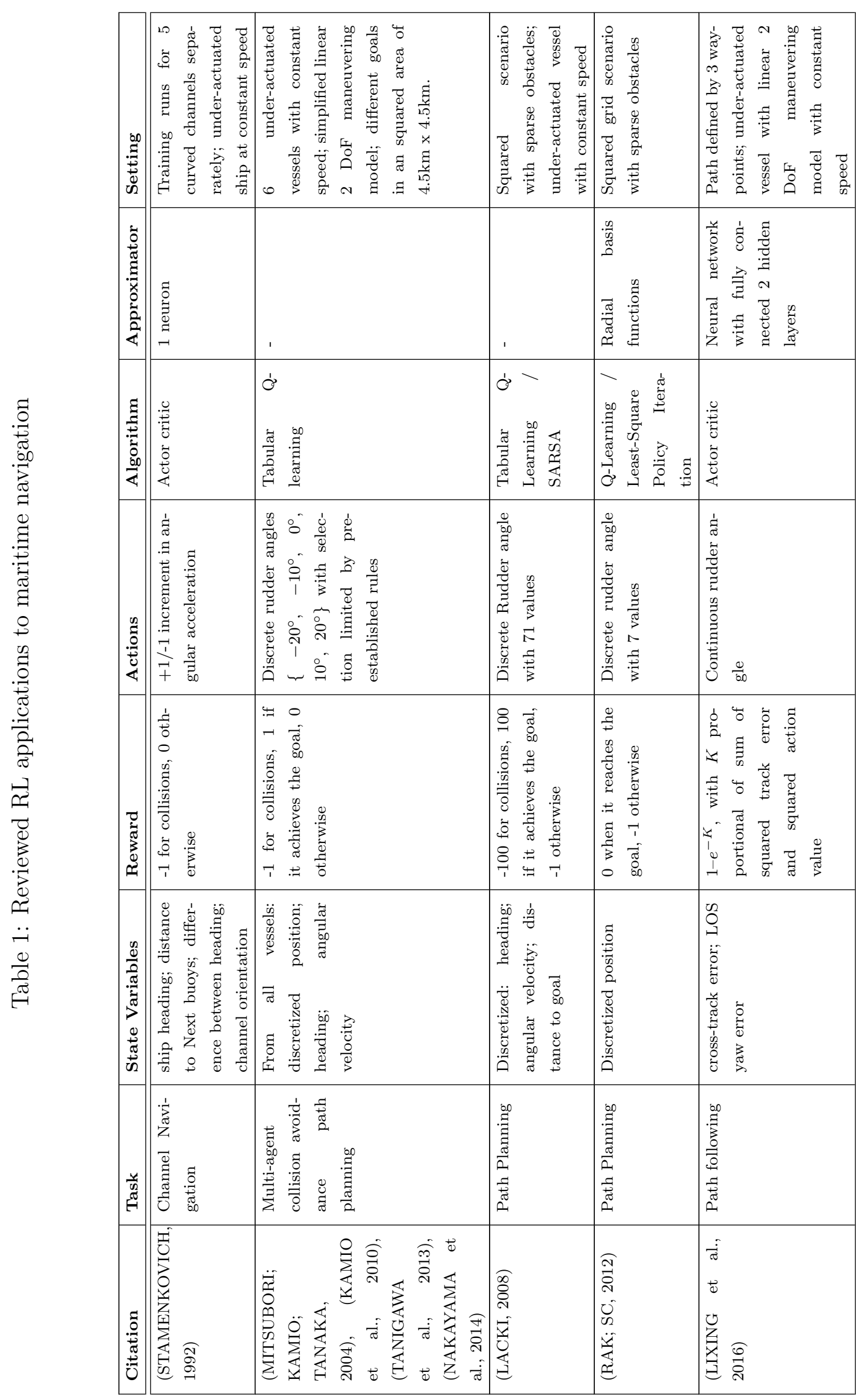




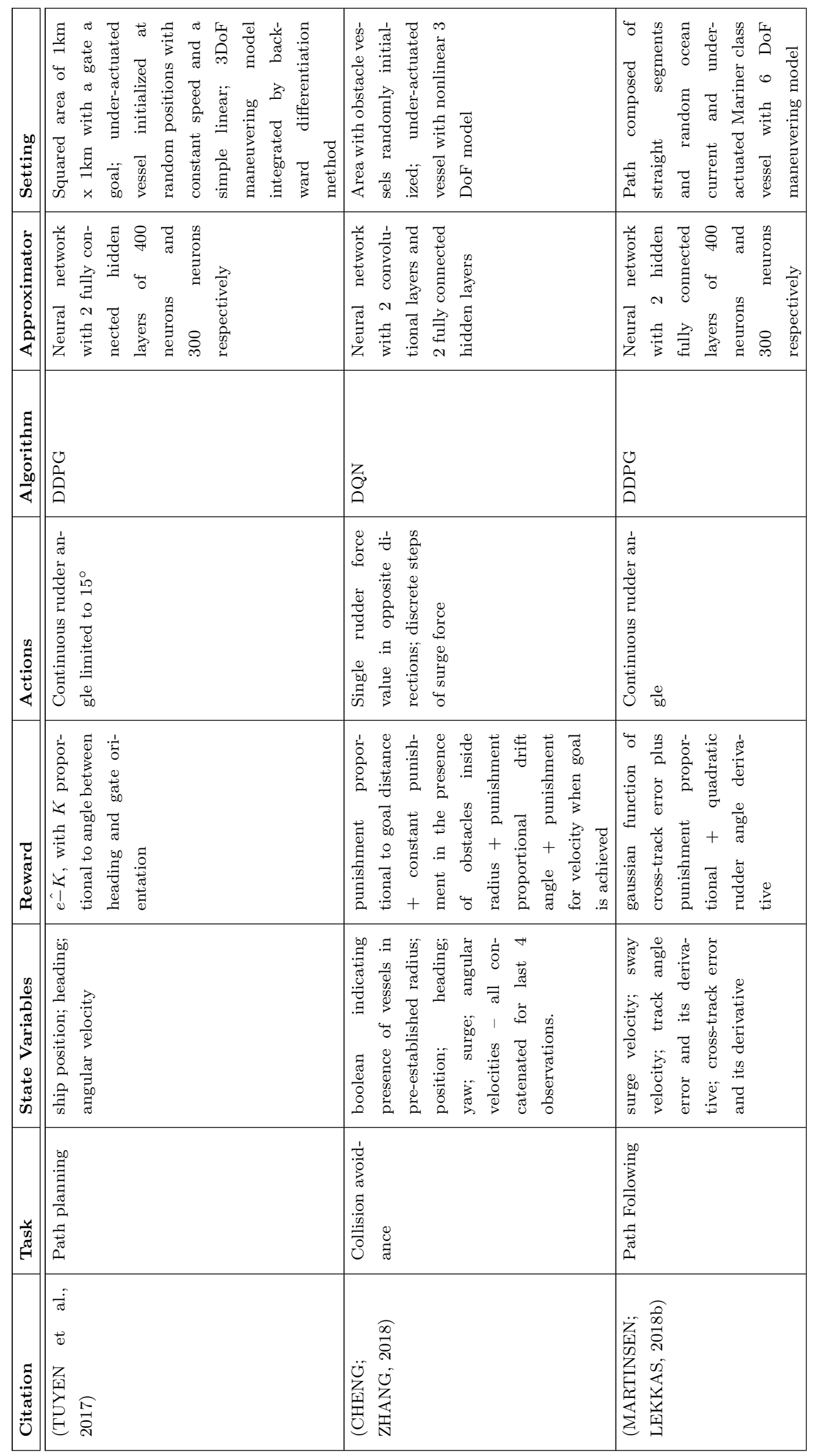




\begin{tabular}{|c|c|c|c|}
\hline & 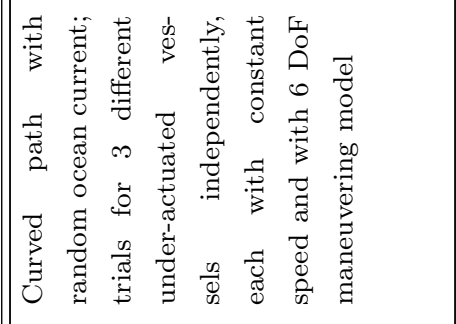 & 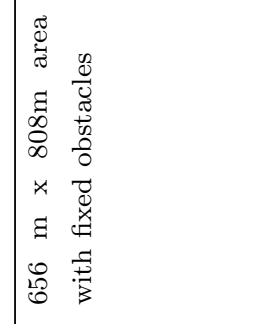 & 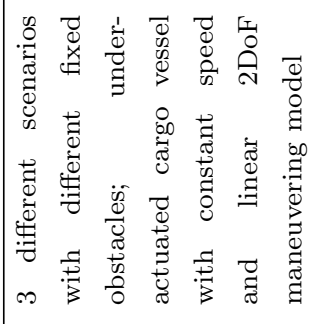 \\
\hline & 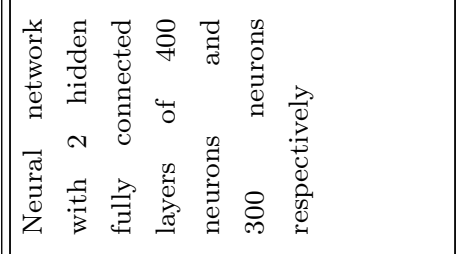 & , & , \\
\hline 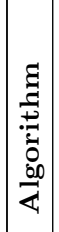 & $\begin{array}{l}0 \\
0 \\
\hat{A} \\
\hat{A}\end{array}$ & 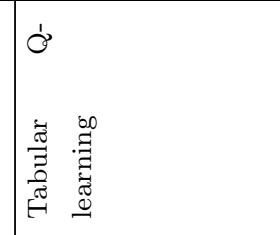 & 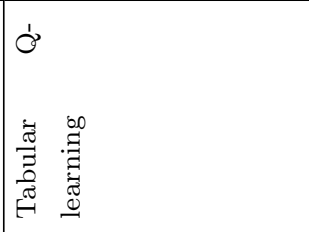 \\
\hline & 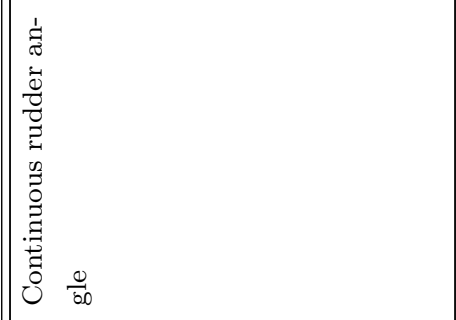 & 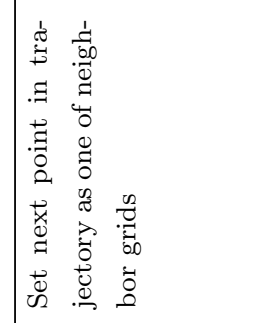 & 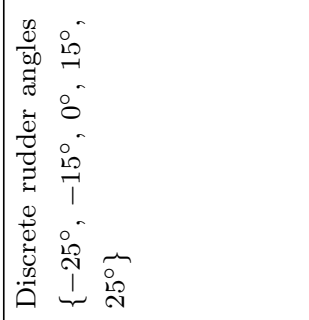 \\
\hline & 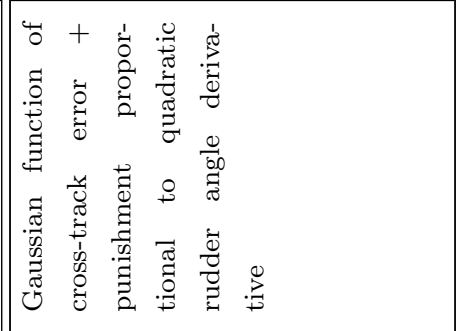 & 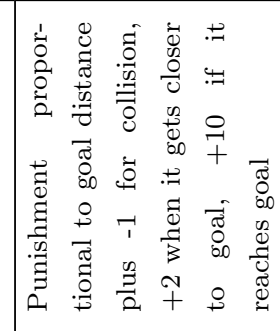 & 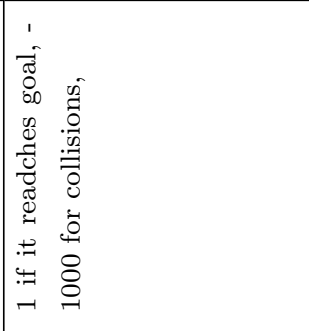 \\
\hline 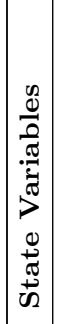 & 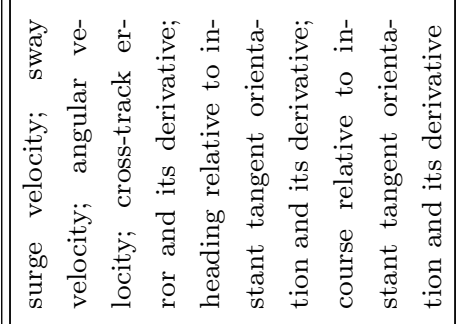 & 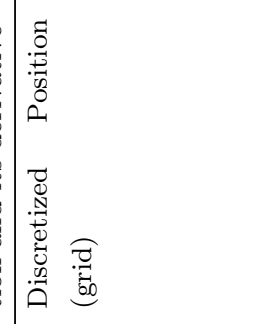 & 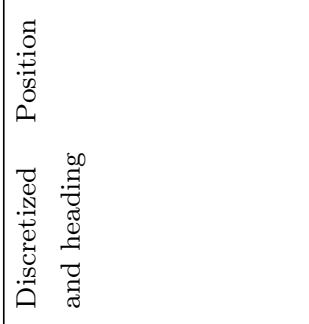 \\
\hline $\mid \begin{array}{c}\frac{y}{n} \\
\tilde{n} \\
\tilde{m}\end{array}$ & 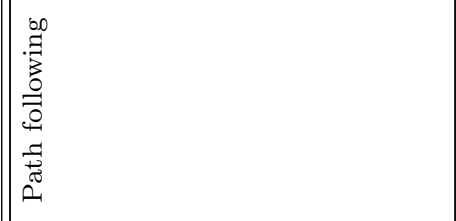 & 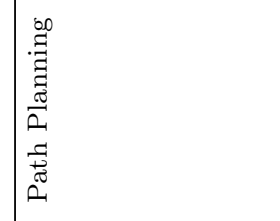 & 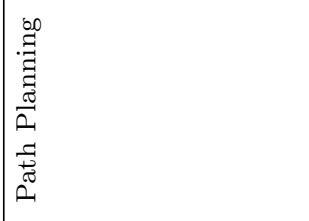 \\
\hline & 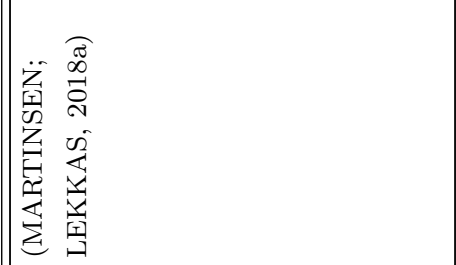 & 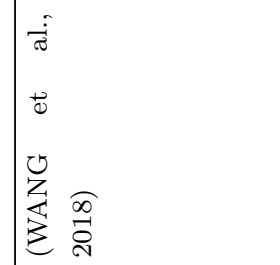 & 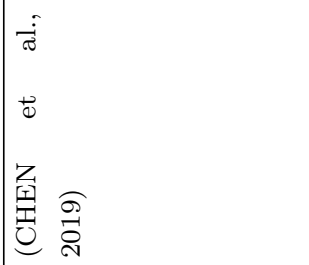 \\
\hline
\end{tabular}




\begin{tabular}{|c|c|c|c|}
\hline 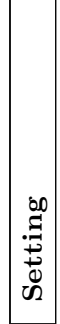 & 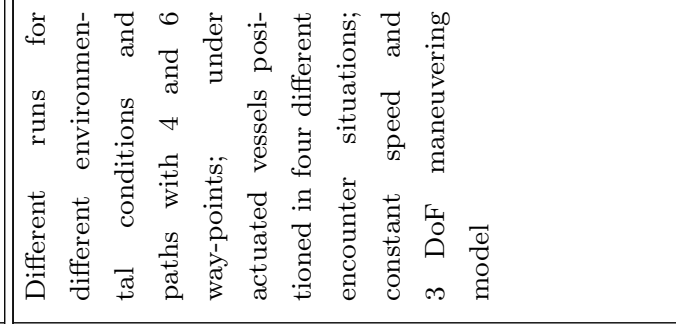 & 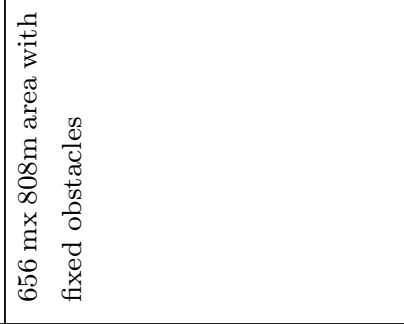 & 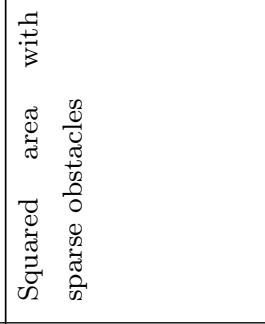 \\
\hline 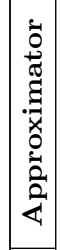 & 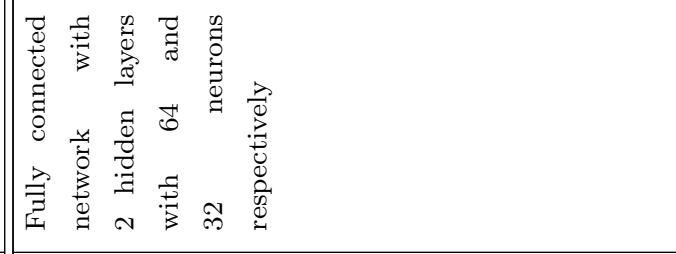 & 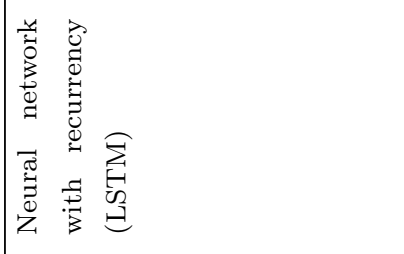 & 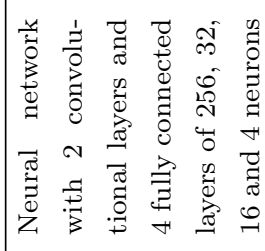 \\
\hline 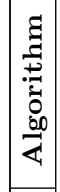 & $\begin{array}{l}0 \\
2 \\
2 \\
2\end{array}$ & 䏍 & 苂 \\
\hline & 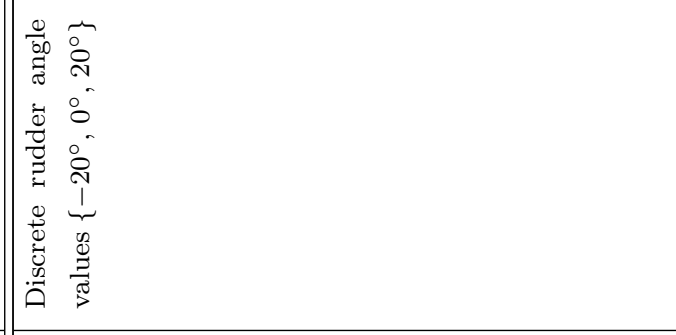 & 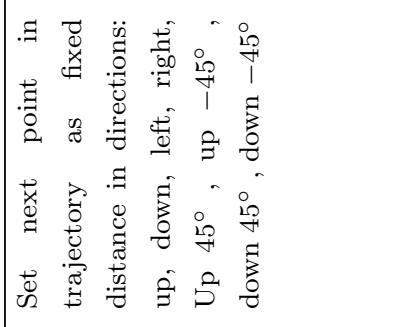 & 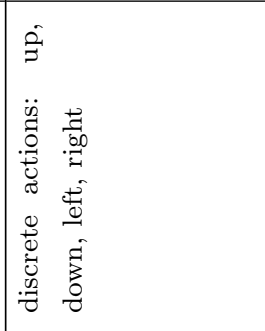 \\
\hline 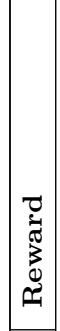 & 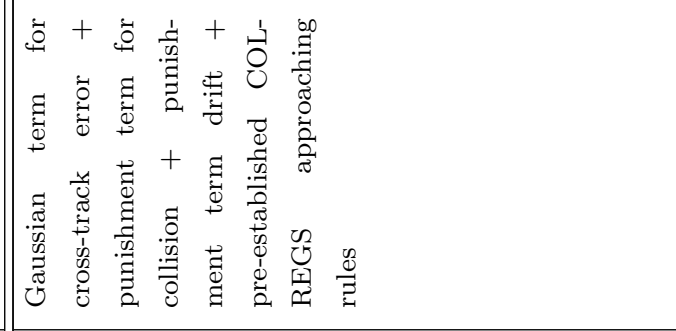 & 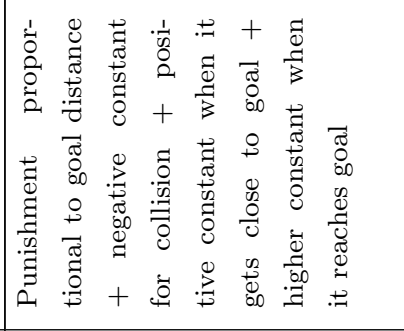 & 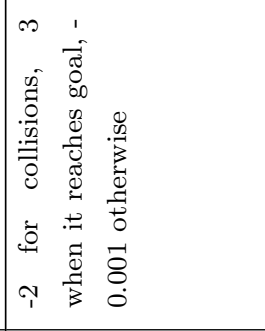 \\
\hline 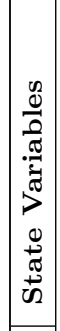 & 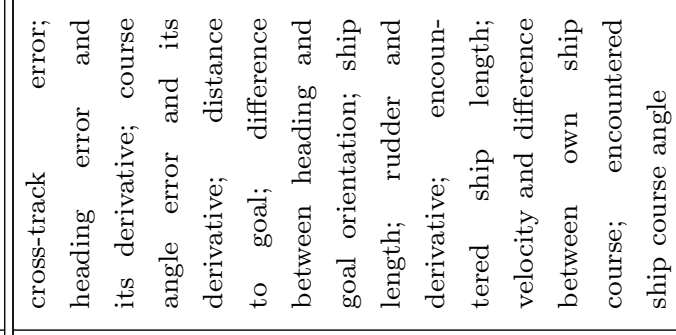 & 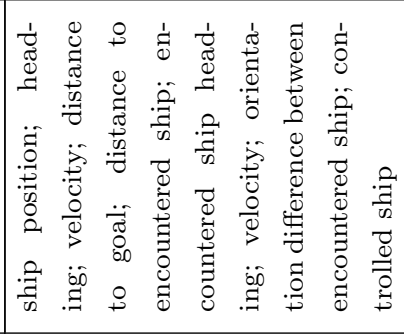 & 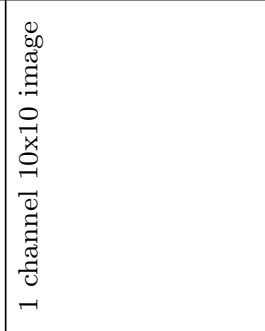 \\
\hline 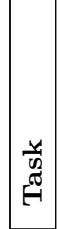 & 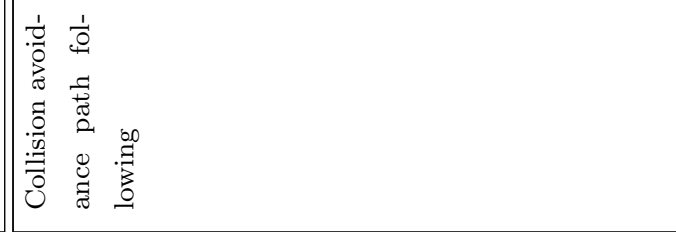 & 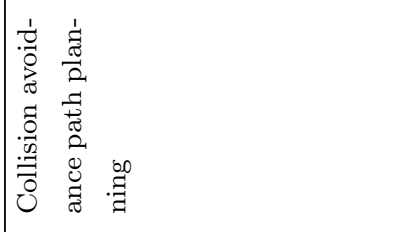 & 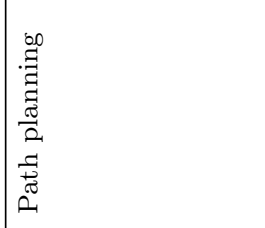 \\
\hline 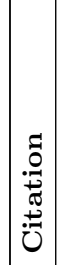 & 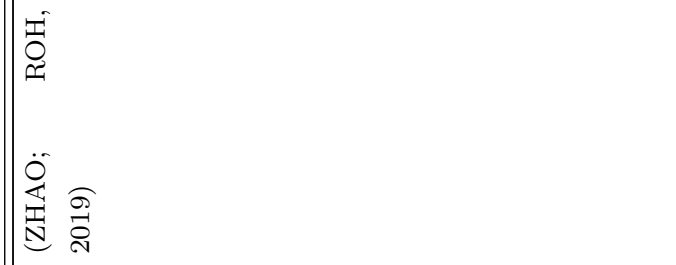 & 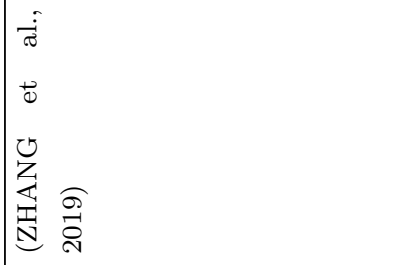 & 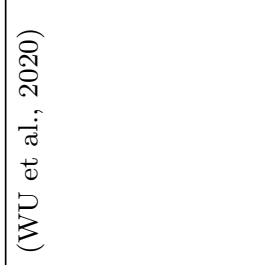 \\
\hline
\end{tabular}




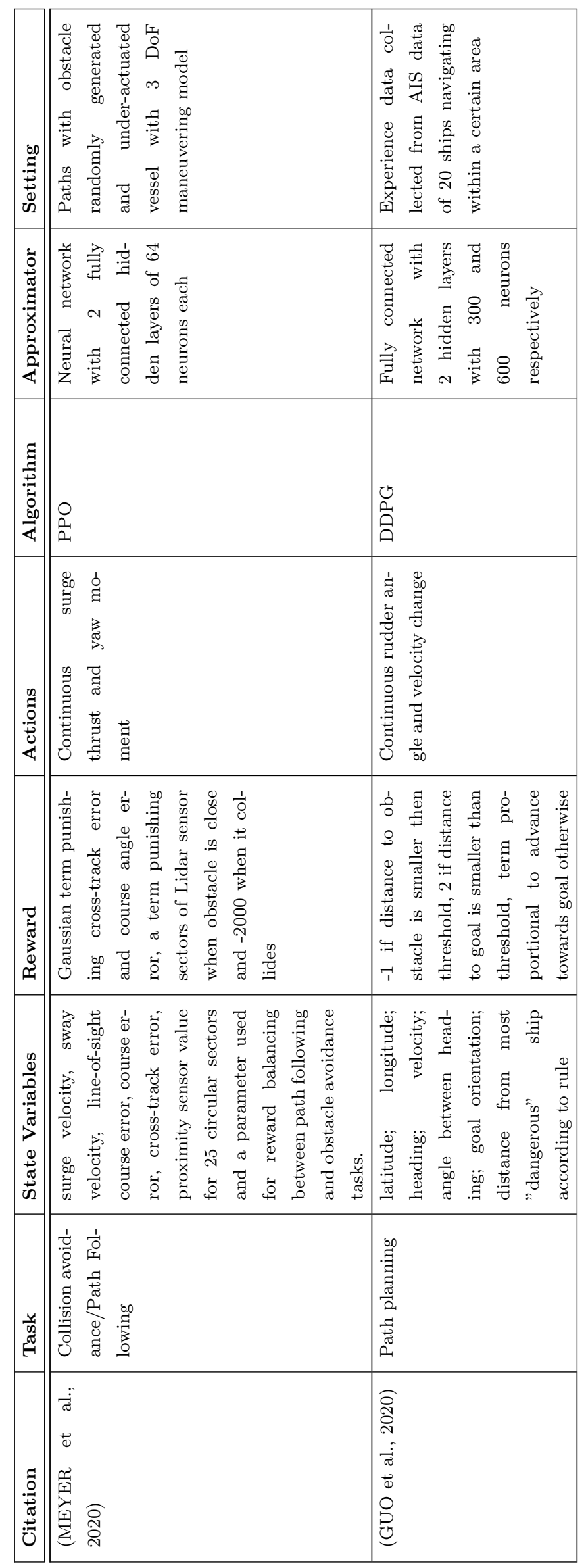




\section{PROPOSAL}

Several issues emerge when applying RL to real-world problems (DULAC-ARNOLD; MANKOWITZ; HESTER, 2019), namely: Training from fixed logs of an external behavior policy; Learning on the real system from limited samples; High-dimensional continuous state and action spaces; Safety constraints that should not be violated; Tasks that may be partially observable or non-stationary; Reward functions that are unspecified or multiobjective; System operators who desire explainable policies and actions; Inference that must happen in real-time at the control frequency of the system; Large and/or unknown delays in the system actuators, sensors, or rewards.

All of the fore mentioned issues should be accounted when considering RL for control of real vessels as any other physical systems. As the scope of this dissertation is limited to fast-time simulations using a high-precision mathematical model, problems such sample limitation, safety constraints and real-time control can be naturally disregarded.

In this proposal, high-dimensionality problem was circumvented by adopting a compact representation that - even partially observable - can still generate satisfactory policies. Capacity of generalization for multiple environmental conditions was also considered in this proposal. The RL task presented does not include conflicting objectives and was simplified to path following and collision avoidance terms. The robustness of this proposal was further evaluated by applying two different RL algorithms.

The state representation is described in Section 4.1; Sections 4.2 and 4.3 describe artifacts that improve generalization capacity of the solution and sample efficiency; Section 4.4 describes the action space adopted; Section 4.5 describes the reward function and finally Section 4.6 and 4.7 explain the two algorithms employed. 


\subsection{State variables}

The state space combines variables that are essential to path-following tasks - which would characterize the problem as a traditional infinite horizon control task - with variables and details that enable the policy to learn behaviors typical from path planning tasks. Table 3 lists all states variables adopted in the representation, and Figure 8 depicts them.

In this setting, the propeller command remains fixed, so the agent does not control velocity. That reduces the dimensionality of the problem, as all variables related to the vessel velocity and longitudinal effects of wind and current are not considered in the state space. Differently from the majority of works applying RL to vessel navigation, the vessel speed is not held constant. As most human pilots perform navigation trough channel with very little or no thrust variations, the assumption is that speed variations due to longitudinal components of wind,current, and the drag force caused by rudder movements should not affect drastically navigation performance.

No information regarding the margins was directly used in the state representation. That makes the solution more robust against variations in margin geometry as long as the training occurs considering the most critical topologies.

Table 3: State variables.

\begin{tabular}{|c|l|l|}
\hline State variable & Unit & Range \\
\hline dist_to_path & $\mathrm{m}$ & -100 to 100 \\
\hline cog_error & $\mathrm{rad}$ & $-\pi$ to $\pi$ \\
\hline rate_of_turn & $\mathrm{rad} / \mathrm{s}$ & -1.0 to 1.0 \\
\hline lat_wind_vel & $\mathrm{m} / \mathrm{s}$ & -8.0 to 8.0 \\
\hline lat_curr_vel & $\mathrm{m} / \mathrm{s}$ & -1.0 to 1.0 \\
\hline dist_to_waypoint & $\mathrm{m}$ & 0 to 1000 \\
\hline
\end{tabular}

The state variable dist_to_path is the distance from the vessel's center point to the desired path segment. In usual path-following tasks, only unsigned distance combined with orientation and angular velocity would suffice as representation. However, when dealing with curves in narrow channel portions, the policy must discriminate at which side of the path the vessel is. As means of differentiation, the value is negative when the vessel is at the left side of the path pointing towards the channel end.

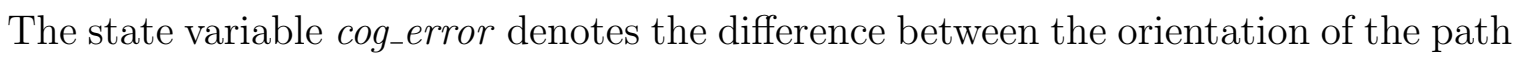
segment and the course over ground (cog). The cog was considered, rather than heading angle, as the vessel can navigate with drift angle to compensate for environmental forces. 


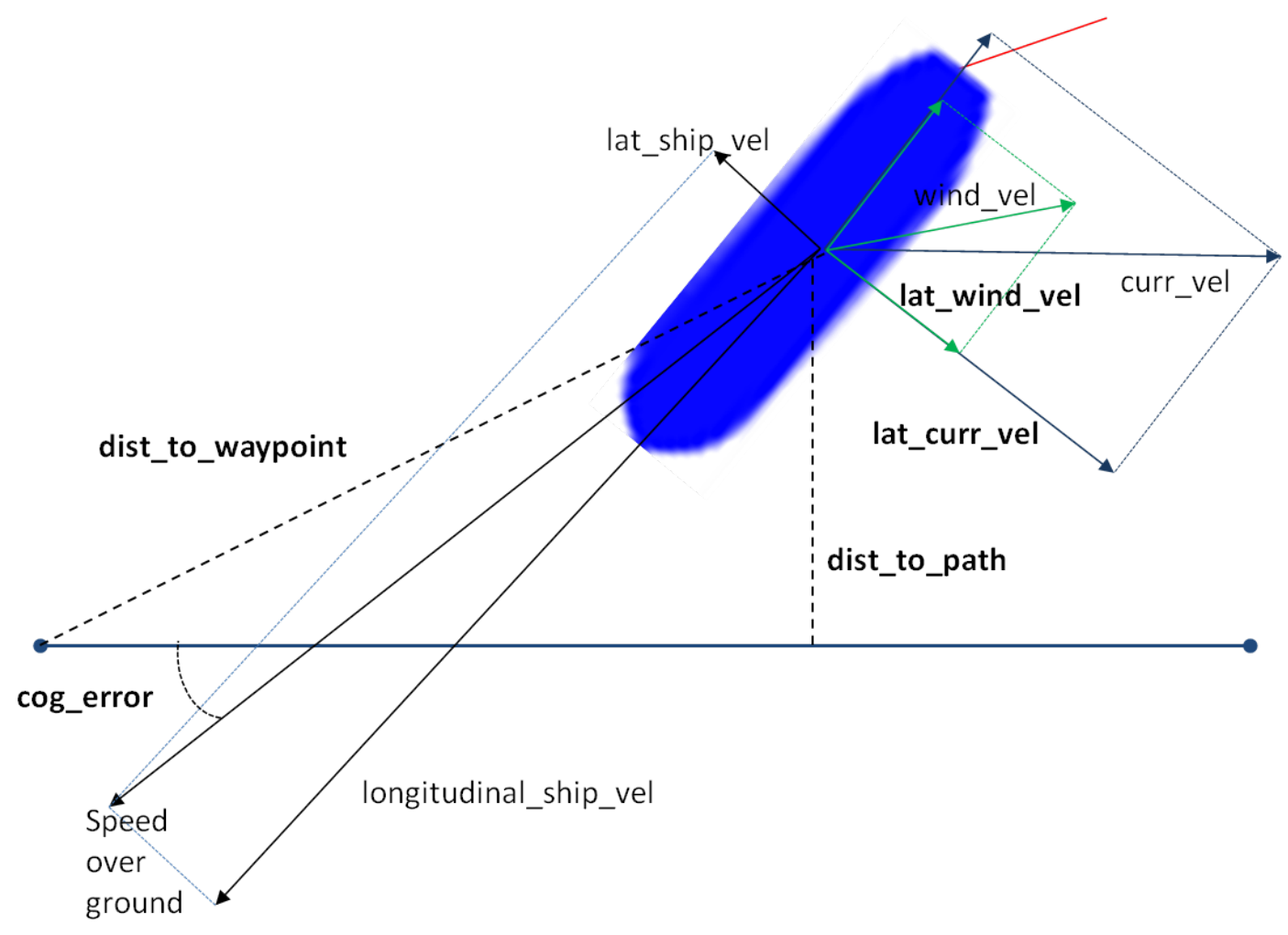

Figure 8: State variables (in bold).

The variable rate_of_turn denotes the angular velocity, which is positive for clockwise rotation and is important due to the high rotational inertia. The variables lat_wind_vel and lat_curr_vel denote the components of wind and water current velocities acting perpendicular to the ship. These variables help the policy to compensate for environmental forces that push the vessel towards the margins.

Finally, state variable dist_to_waypoint carries information on how far is the vessel from the next way-point; It enables the policy to take actions that anticipate changes in $\operatorname{cog}$.

As suggested by (BHATT et al., 2019), normalization of state variables so that they vary in a similar range can enhance learning performance. The variables were normalized between their extreme values observed in prior experiments. The dividing factors for variables can be found in Table 4.

On one hand, adoption of a compact representation, ignoring some variables judged as non-essential, tackles high dimensionality in state space. On the other hand, that drives the solution towards another known issue: partial observability and non-stationarity. When partially observable MDPs are treated as correct MDPs, theoretical convergence to an optimal policy gets compromised. Nonetheless, a reasonable policy that accomplishes the desired goal can still be obtained if training occurs in randomized conditions and the 
Table 4: Dividing Factors.

\begin{tabular}{|c|l|}
\hline State variable & Dividing Factor \\
\hline dist_to_track & 100 \\
\hline cog_error & $0.15 \pi$ \\
\hline rate_of_turn & $0.0015 \pi$ \\
\hline lat_wind_vel & 4 \\
\hline lat_curr_vel & 0.2 \\
\hline dist_to_waypoint & 1000 \\
\hline
\end{tabular}

learning error for those is averaged. In the solution proposed, experiences under different channel portions and environmental conditions play a key role in order to accomplish successful trajectories with reduced number of state variables.

\subsection{Task Segmentation and Context Variable}

Initial experiments with straight narrow channels indicated a difficulty with the states described in the previous section. An experiment was executed in a channel with two $90^{\circ}$ curves to opposite directions separated by a straight segment. The vessel was expected to track the centerline segments generated by the channel margin. The policy learned to navigate in the first curve and to keep track of the straight middle segment, but failed in the second curve. That happened because the state-space representation did not include information about the vessel position relative to the extent of the channel nor information about the curvature. As the policy could not distinguish any difference between these situations, it could not learn to take actions accordingly.

If the policy simply tracks the closest segment, it eventually starts to track another segment highly misaligned, causing collision with channel margins. Thus, the learned policy needs to take crucial actions during the first straight segment in order to anticipate the first curve.

This problem is illustrated in Figure 9 (top). That issue could be solved by tying solutions to specifics of each part of the channel. However, the policy for certain channel parts would no longer be applicable to other similar parts (e.g. straight parts of a channel would not no longer be handled by same representation due to different positions along the channel). Figure 9 (bottom) illustrates this issue.

The solution found to achieve a robust policy was, first, to segment the navigation in different RL episodes, and, second, to parameterize the $\mathrm{Q}$ function by a context variable. 

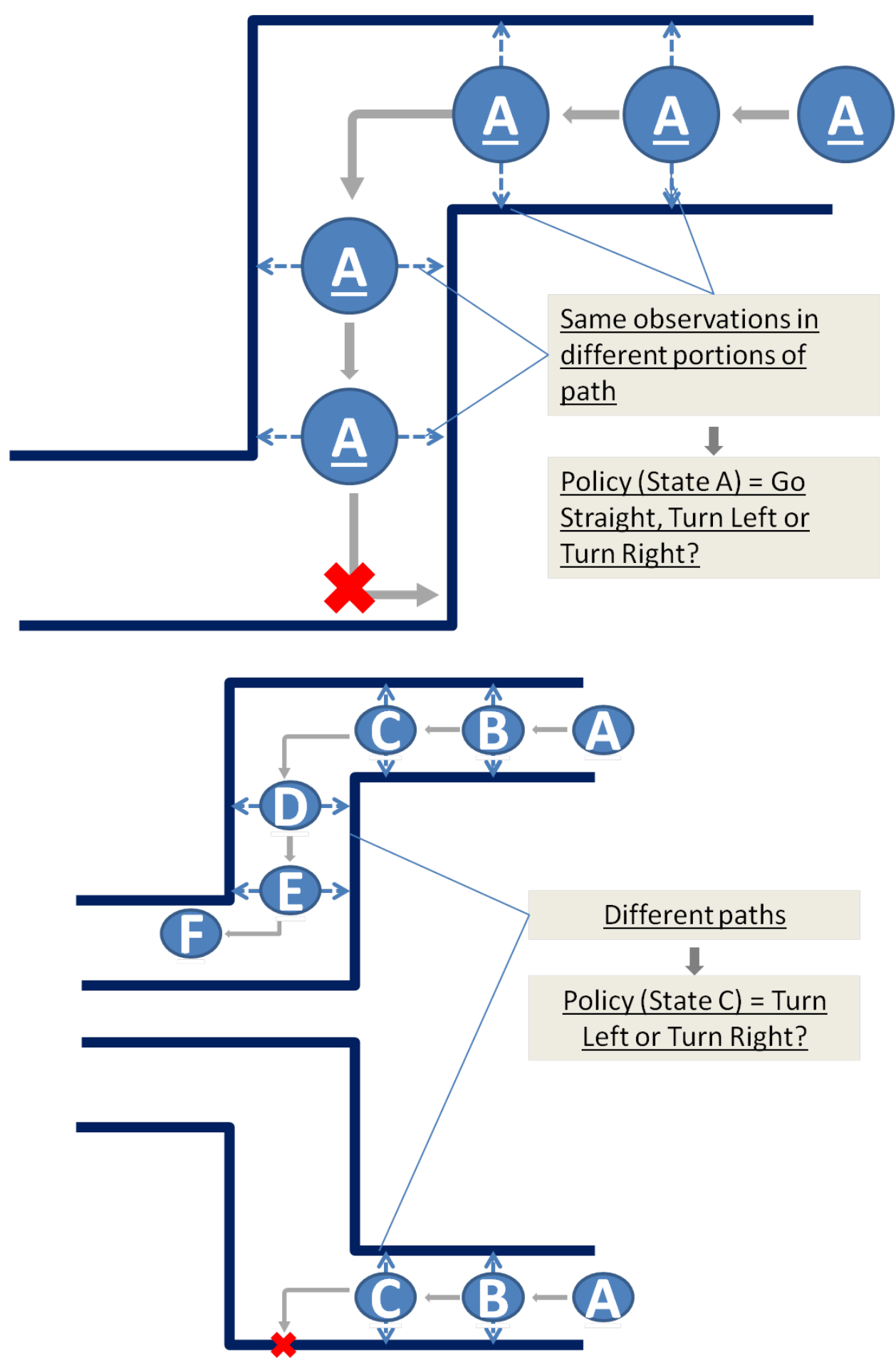

Figure 9: Top: a compact representation with no information about vessel's position may "confuse" the policy. Bottom: Vessel's position included in the state representation prevents the reuse of policy in different parts of the channel.

Episode Segmentation: Each RL episode is delimited by three way-points in the channel centerline with a fixed distance $L_{\text {segment }}$ between them. That creates two desired path segments that the vessel must track, as seen in Figure 10. Instead of channel center lines, those segments are used as reference in order to standardize episode length span 
and to improve learning efficiency. The distance $L_{\text {segment }}$ cannot be too short so as to avoid trajectory instability in face of small angles, and it cannot be too long so as to avoid reducing the navigable area. The episode ends as soon as the vessel follows the two desired path segments and reaches the second waypoint ahead. The segmentation improves sample efficiency, as experiences from a portion of channel can be used for similar situations in any channel position.

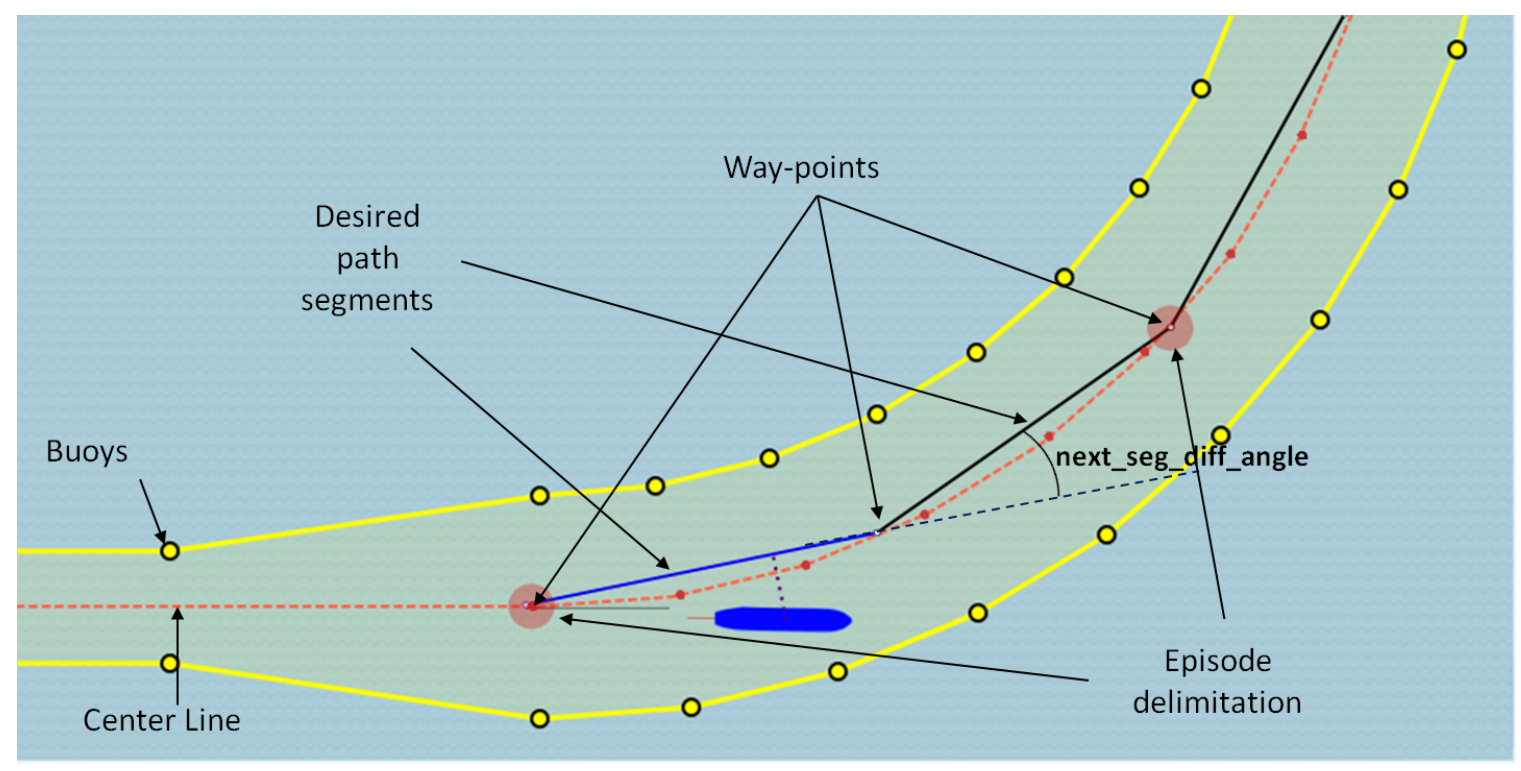

Figure 10: Channel partitions.

Context Variable: Pursuing two path segments with different angles under environmental forces demands a policy that succeeds in a diversity of situations. The higher the angle between path segments, the more the agent must anticipate the rudder action before finishing the actual segment. Context variables were included in the Q-value function to discriminate the context of the episode that the agent is facing. This RL technique was proposed by (SCHAUL et al., 2015); it consists of extending the input of the value function with information about the context of the task being performed by the agent, so $Q(s, a)$ becomes $Q(s, c, a)$. This enables multi-task generalization as the policy can use information that tells which task it must accomplish in a given episode. In this proposal, the context variable is the smallest difference between the orientation of path segment ahead of the next way-point and the orientation of the desired path segment being followed, also referred as next_seg_diff_angle in Figure 10 (for segments at the end of the channel, this angle is set to zero). 


\subsection{Symmetry}

So as to improve sample efficiency, a symmetric property of the formulation was assumed. For regular path following tasks, it would be more intuitive to adopt one side of the path as "mirror" from the other side; The policy would need to learn from experiences from one side only and would invert signals from state variables and from action if the vessel were at the other side. However, it is expected that the policy adopts anticipating behavior in order to navigate through curves, so actions cannot simply be "mirrored" based only in the symmetry in relation to the path. In this case, the curve must also be accounted and the symmetry must consider pairs of segments, as illustrated in Figure 11. When the curve turns right (meaning, the context variable $c<0$ ), both context variable and all other state variables, with exception of the variable dist_to_waypoint, have their signals inverted. It is equivalent to say that the policy learns to navigate in curves that turn left and can be reused to curves that turn right only by signal shifting.

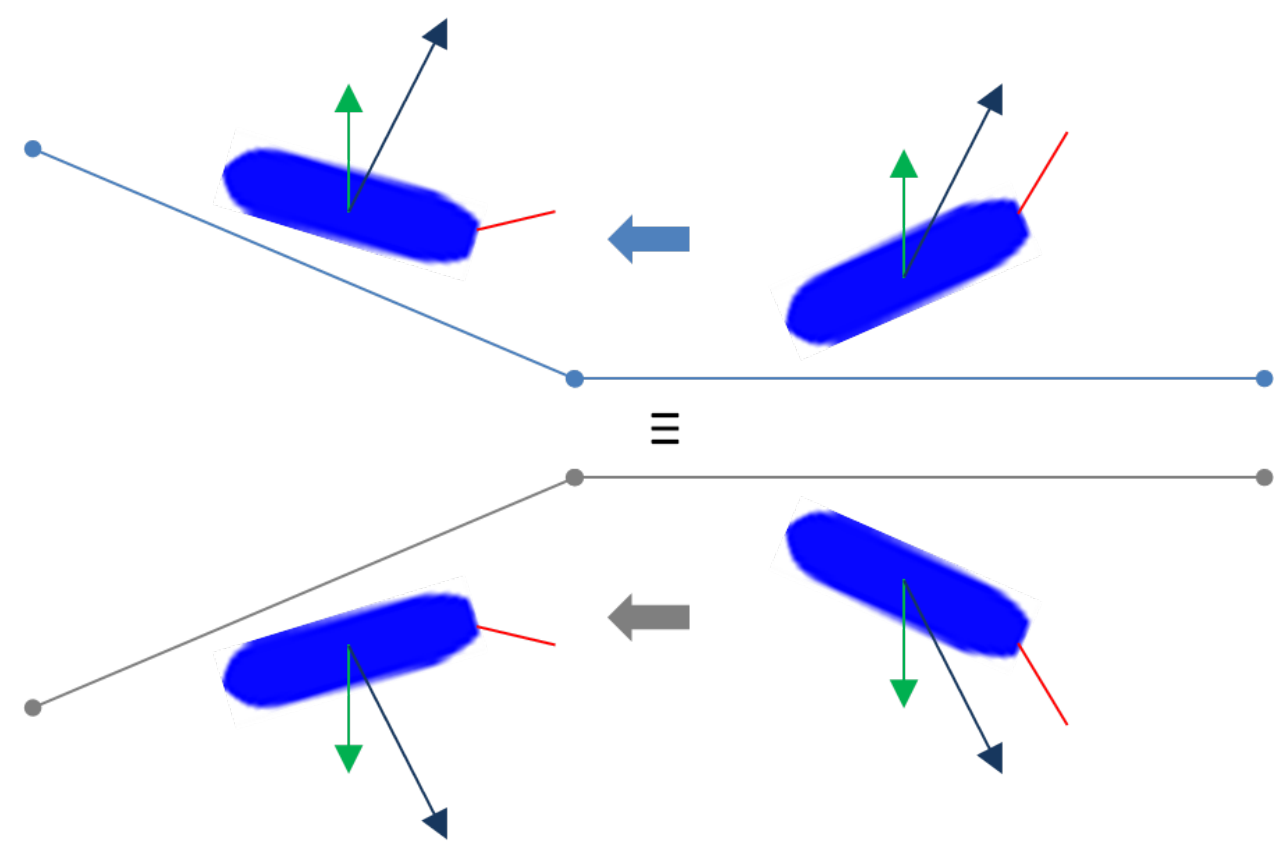

Figure 11: Symmetry of experiences for pair of segments

\subsection{Actions}

In order to better represent human piloting, rudder commands are given in discrete values and with minimum time interval between them. While the propulsion level is fixed at $60 \%$ of maximum power along all trajectory, the action values represent the rudder 
command and are normalized by the maximum rudder angle $\left(35^{\circ}\right)$. The rudder levels used were: $-50 \%,-20 \%, 0 \%, 20 \%, 50 \%$. The choice was made based on values that approximate commands given by human pilots and similar criterion was also used in (NAKAYAMA et al., 2014), (CHEN et al., 2019) and (ZHAO; ROH, 2019). Actuation delay is known and accounted in this formulation, as the actual rudder response time to a command is included in the vessel mathematical model.

Differently from other related works reviewed, the time interval chosen for transitions is $T_{\text {transition }}=10 \mathrm{~s}$. That means the vessel remains with the same commanded rudder level for that amount of time and the simulator advances numerical integration steps several times to complete a single experience. The large time span was adopted to impose a constraint in controllabity and make trajectories more likely to be feasible in real-world navigations practiced by human pilots.

\subsection{Reward}

As in RL path planning tasks reviewed in Chapter 3, it is straight-forward to consider negative reward values for experiences where collision with margins occurs. Nonetheless, that is not sufficient to avoid reward sparsity and extremely slow learning process. Since the centerline of the channel is being adopted as reference to the state observations, a reward term similar to path following problems was employed in the formulation.

(RANDLØV; ALSTRØM, 1998) argued that negative values prevent the agent from going through states unnecessarily to accumulate positive rewards. Nonetheless, in previous work (AMENDOLA et al., 2018), reward functions with negative values for most state-space led the agent to quickly go for collisions in order to minimize overall negative punishment. As limited available space in a channel prevents the vessel from navigating away from the centerline, positive rewards are not an issue.

Thus the reward function adopted was:

$$
R\left(s, a, s^{\prime}\right)=R_{\text {cog }} R_{\text {dist }} R_{\text {rot }}+R_{\text {collision }}
$$

ensuring that reward is negative only for collision states. Factors are multiplied to provide a more steep decrease of reward as states become undesirable and the reward terms are defined as follows:

$$
R_{\text {cog }}=\frac{1}{(1+K \mid \text { scaled_cog_displacement } \mid)}
$$




$$
R_{\text {dist }}=\frac{1}{(1+K \mid \text { scaled_distance_to_track } \mid)}
$$

and

$$
R_{\text {rot }}=\frac{1}{(1+K \mid \text { scaled_rot } \mid)},
$$

as they behave in the same way as the vessel moves away from the track and starts rotating respectively. If the vessel collides, a -10 punishment value is added to the function:

$$
R_{\text {collision }}=\left\{\begin{array}{rcc}
-10 & \text { if } \quad \text { ship_collided } \\
0 & \text { otherwise. }
\end{array}\right.
$$

The idea behind this reward structure choice is to keep the terms $R_{\text {cog }}, R_{\text {dist }}, R_{\text {rot }}$ as well as their overall product in $] 0.0 ; 1.0]$ interval. That improves learning stability (HENDERSON et al., 2018) and makes reward more intuitive.

The negative term adopted in $R_{\text {collision }}$ was set with a much higher magnitude than the others so each collision cases highly negative updates in value estimation, increasing learning efficiency. Although -10 was chosen, other values could also be tested in experiments.

Some works reviewed in Chapter 3 limited reward to this interval adopting gaussian functions. However, a gaussian function would demand a more complicated tuning process in order to make an abrupt reward decrease for small vessel deviations from desired path an, at the same time, present informative reward variations as the vessel moves away toward the margins.

To understand the effect of parameter $K$, preliminary experiments demonstrated that $K=10$ improves the punitive behavior when vessel deviates from desired states; thus $K=10$ was adopted. Figure 12 uses the example of rate_of_turn to show how the factor $K$ affects the reward term. The reward term function is plotted with $K=1$ and a more punitive factor $K=10$.

\subsection{APE-X-DQN}

The first algorithm employed extends DQN with Distributed Prioritized Experience Replay (APE-X). Its architecture was proposed by (HORGAN et al., 2018) and is illustrated in Figure 13. APE-X-DQN is an off-policy value-based algorithm. One advantage of adopting this type of algorithm is that the estimation of values for state-action pairs can also be useful in future applications for fast-time simulations in maritime domain, 


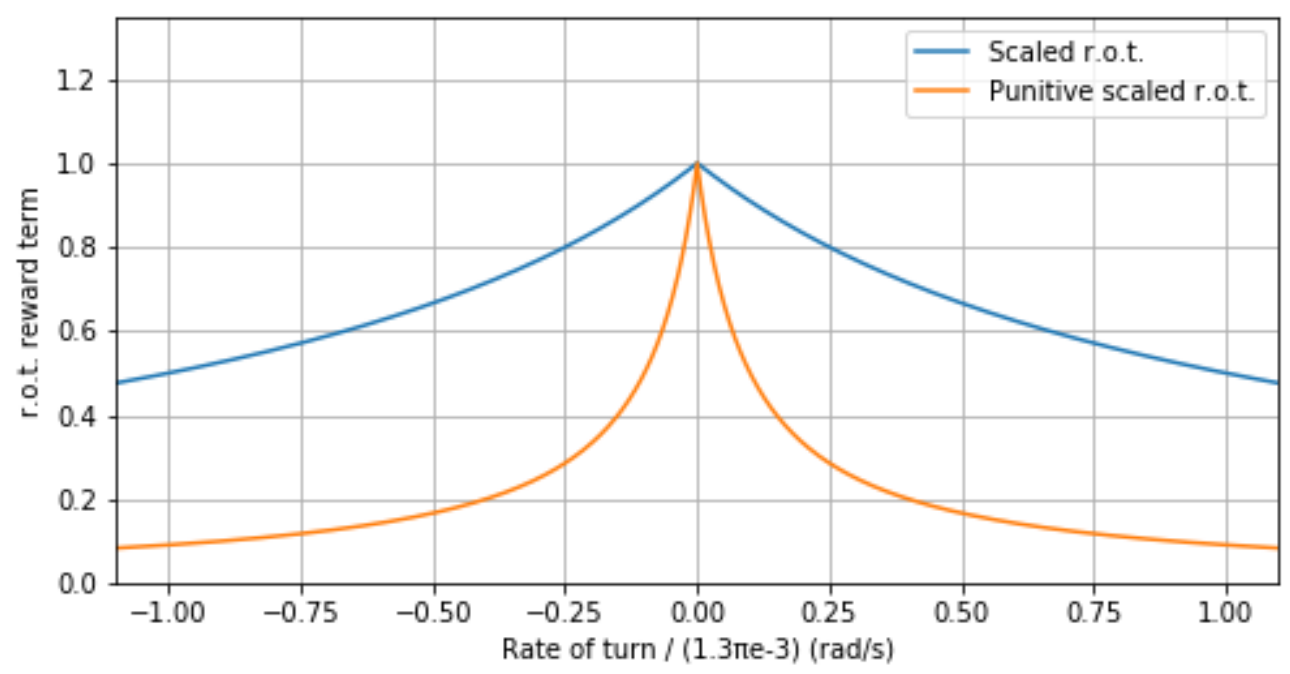

Figure 12: Effects of rate_of_turn punishment; $K=1$ for Less Punitive Behavior; $K=10$ for Punitive Behavior (adopted value).

when inspired by ideas from robotics (CRUZ et al., 2018; MOERLAND; BROEKENS; JONKER, 2018). It consists of two entities that share a Replay Memory Buffer: Actor and Learner. The multiple actor instances are responsible for interacting concurrently with separate environment copies that, in this case, are instances of the fast-time simulator. The role of each actor is to evaluate a policy and to store the experiences observed in a replay memory buffer; its policy is implemented as a deep neural network. The second part of the architecture consists of a learner that is responsible for sampling batches of data from the replay memory buffer to update the policy parameters. The learner periodically communicates updated network parameters to the actors. The replay memory buffer is centralized and shared between the actors - who feed it - and the learner - who consumes its data.

An actor is instantiated in each computing core available. The instance contains a copy of the policy neural network and its function is to collect experiences by interacting with fast-time simulator instances. Actors explore different parts of channel under different current and wind conditions concurrently. In order to collect experiences from multiple scenarios and/or conditions independently of the number of computing cores available, the implementation allows each actor's policy to interact with multiple simulator instances configured differently, collecting an experience for each instance sequentially.

The collected experiences consist of the state $s$ where an action was performed, the action $a$ itself, the state $s^{\prime}$ obtained after action, the reward signal $r$, the context variable $c$ and the end_flag, indicating whether the state $s^{\prime}$ is final in the episode. 


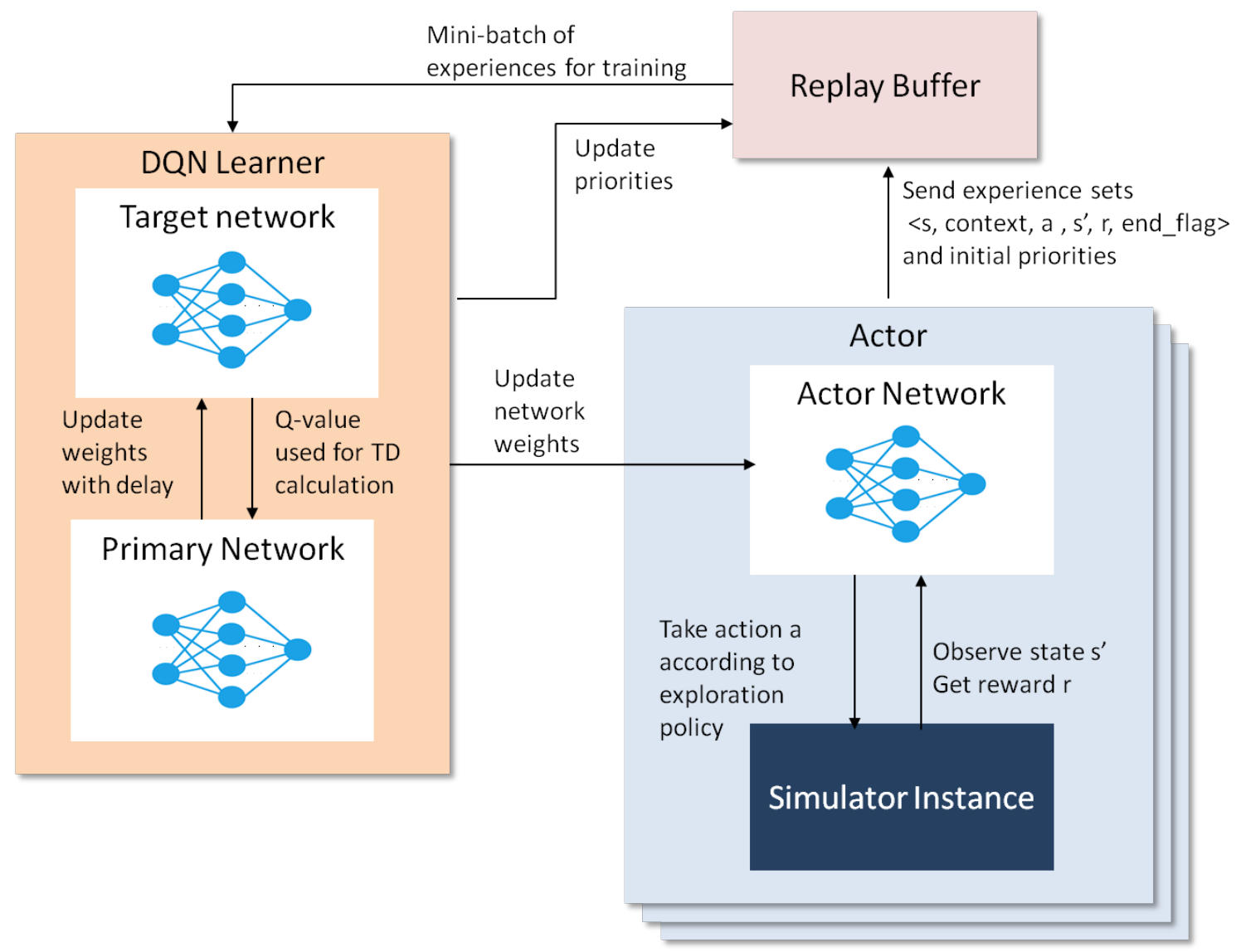

Figure 13: Architecture: APE-X-DQN.

The experience set $<s, a, s^{\prime}, r, c, e n d_{-} f l a g>$ of an actor is sent to an actor local buffer. Batches with $b$ experiences from the local buffer have their initial priority value calculated locally and then are sent to the replay memory buffer. The initial priority for a given experience is calculated based on its TD error using the actor network, as:

$$
\delta_{\text {actor }}=r_{t}+\gamma \max _{a} Q\left(s_{t}, c_{t}, a, \theta_{\text {actor }}\right)-Q\left(s_{t}, c_{t}, a, \theta_{\text {actor }}\right),
$$

where in time step $t, \delta_{\text {actor }}$ is the TD error, $r_{t}$ is the reward, $s_{t}$ is the state, $c_{t}$ is the context, $a_{t}$ is the action, $\gamma$ is the discount factor and $\theta_{\text {actor }}$ represents the actor network parameters. The priority is then calculated as:

$$
p_{e}=\left(\delta_{a c t o r}(e)+k_{p r}\right)^{\alpha_{p r}}
$$

where $p_{e}$ is the priority for a given experience $e, k_{p r}$ is a small positive constant that prevents the edge-case of transitions not being revisited when their TD error is zero and $\alpha_{p r}$ is a factor that determines how much prioritization is used, with $\alpha=0$ representing the uniform case where all experiences have the same probability of being sampled. 


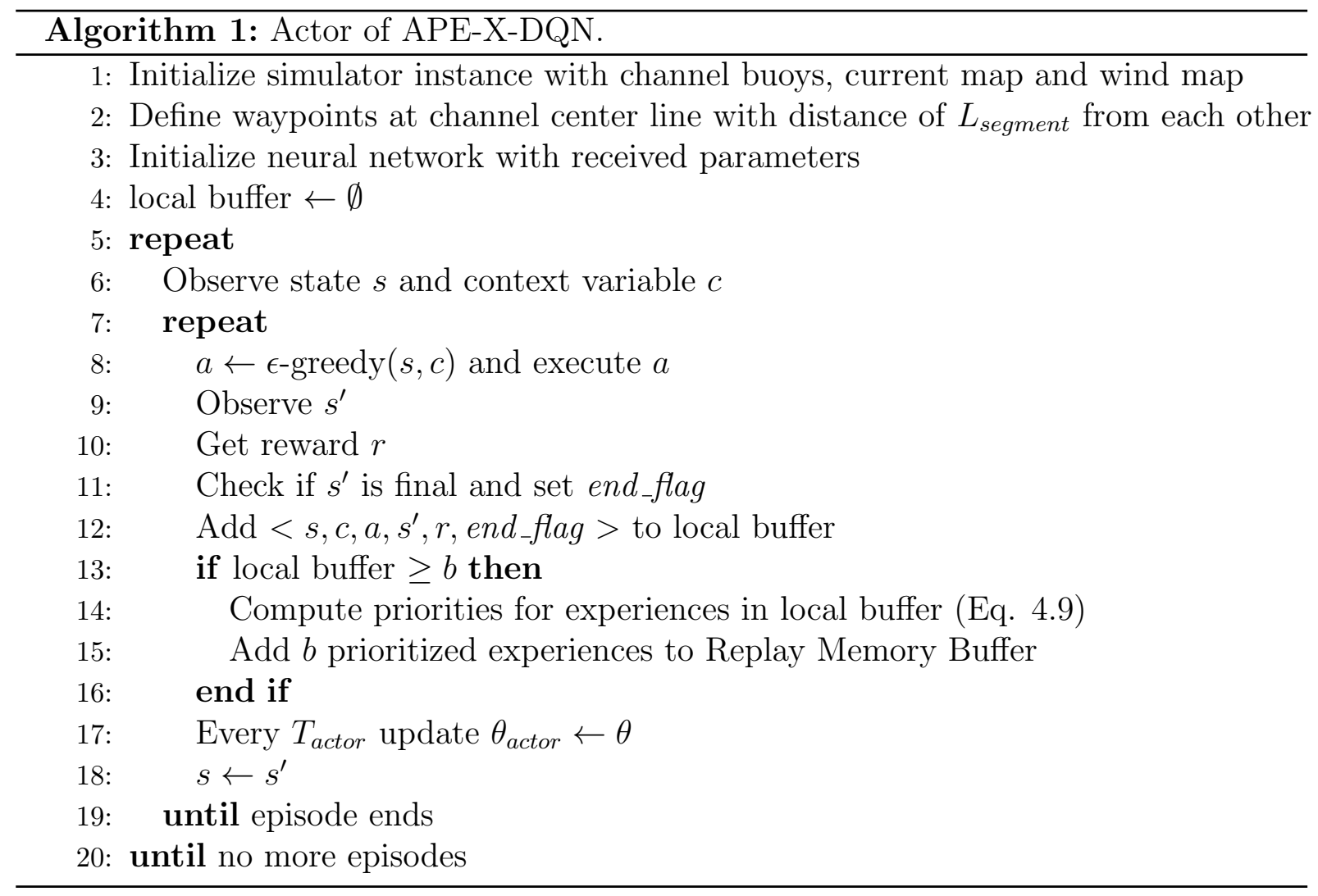

The actor's policy network parameters $\theta_{\text {actor }}$ are synchronized with the primary network parameters at every $T_{\text {actor }}$ experiences collected from it. Each episode ends either if the vessel collides, reaches the end of the channel or finishes tracking two path segments. In order to balance the learning process throughout the channel, actors initialize the vessel at their simulator instance randomly in one of the defined way-points after the vessel collides or reaches the end of channel. The exploration policy adopted by the actors was the $\epsilon$-greedy strategy. The actors act while the training is carried out by the learner. Algorithm 1 describes the interaction between each actor and the simulator instance.

The learner in the APE-X-DQN architecture employs the DQN algorithm (Mnih et al., 2015) and is composed of a deep neural network that estimates Q-values for the policy. The network scheme is illustrated in Figure 14.

The learner periodically sends the actors the weights of this neural network so that they can update their respective neural networks. The training of the learner occurs in mini-batches $B$ of experiences sampled from the Replay Memory Buffer with size $D$.

In order to prioritize training for experiences that are more "surprising" (HORGAN et al., 2018), the probability of an experience set being sampled from the replay memory buffer to a batch is modulated by its priority factor. The experience priority is updated by the learner primary network. The update is also performed using TD calculation, as 


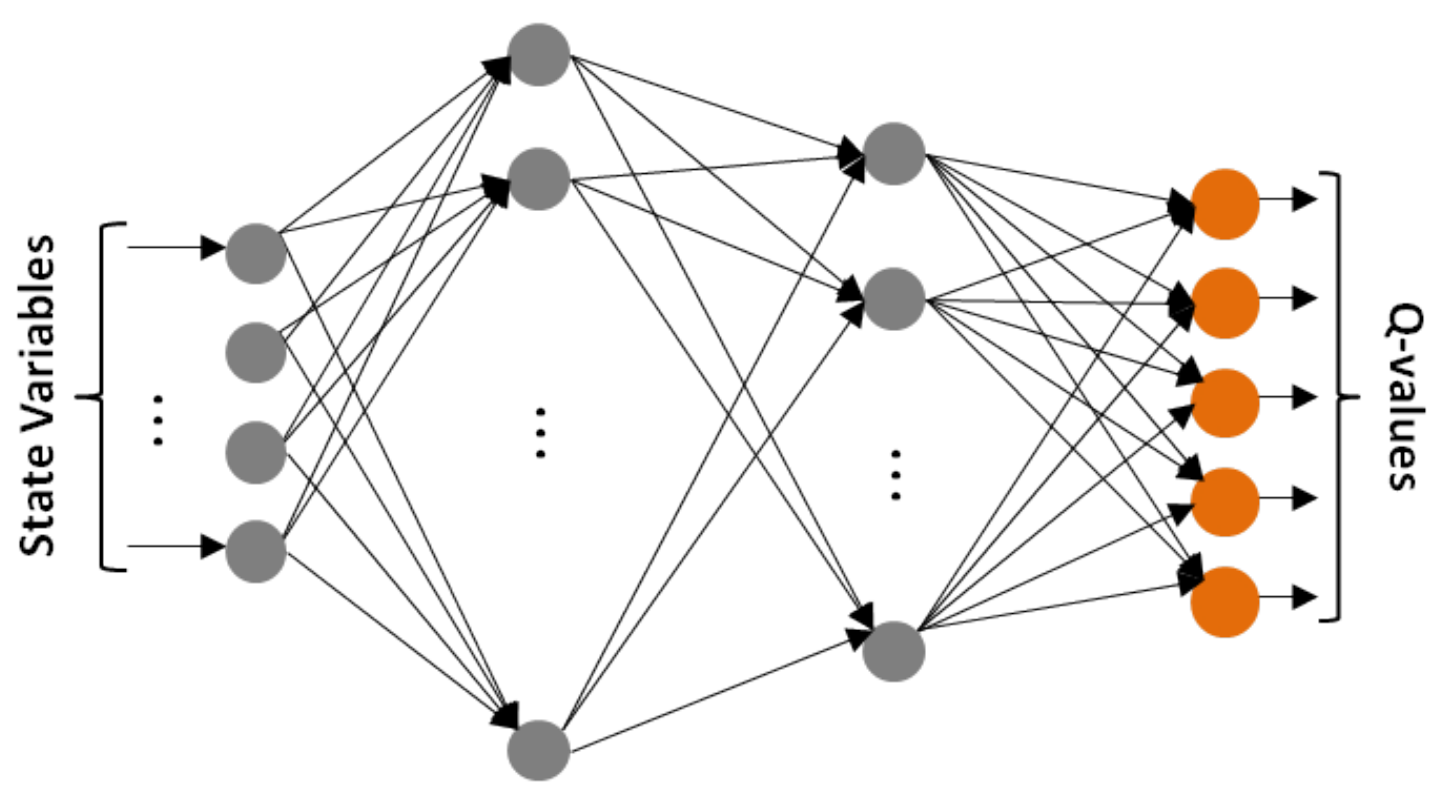

Figure 14: Neural network architecture for APE-X-DQN policy

in Equation 4.7, but employing the primary network parameters $\theta$ instead, as:

$$
\delta=r_{t}+\gamma \max _{a} Q\left(s_{t}, c_{t}, a, \theta\right)-Q\left(s_{t}, c_{t}, a, \theta\right)
$$

The probability of a given experience $e$ being sampled is then given by:

$$
P(e)=\frac{p_{e}}{\sum_{n=1}^{D} p_{n}},
$$

where $p_{e}$ is the priority for experience $e$ calculated with $\delta$. Prioritized replay introduces bias because it changes this distribution of updates, and therefore changes the solution that the estimates will converge to. That can be corrected by introducing a importancesampling (IS) weight to the TD error of a given experience $e$, given by:

$$
w_{e}=\left(\frac{1}{D P(e)}\right)^{\beta},
$$

where $D$ is the buffer size, $P(e)$ is the probability of sampling $e$ calculated in Equation 4.9 and $\beta$ is the correction factor. If $\beta=1$, the bias for non-uniform sampling distributions is fully compensated.

For computational efficiency purposes, the probability density function is approximated by storing priority values in a "sum-tree" data structure, which consists basically of a binary tree, where each parent node is the sum of its children and leaf nodes store the transition priorities and the internal nodes are intermediate sums. For a batch of $B$ experiences being sampled, the sum of all priorities is divided in equal intervals of size $B$ and a value from sum-tree is uniformly sampled inside each interval. The experience sets 


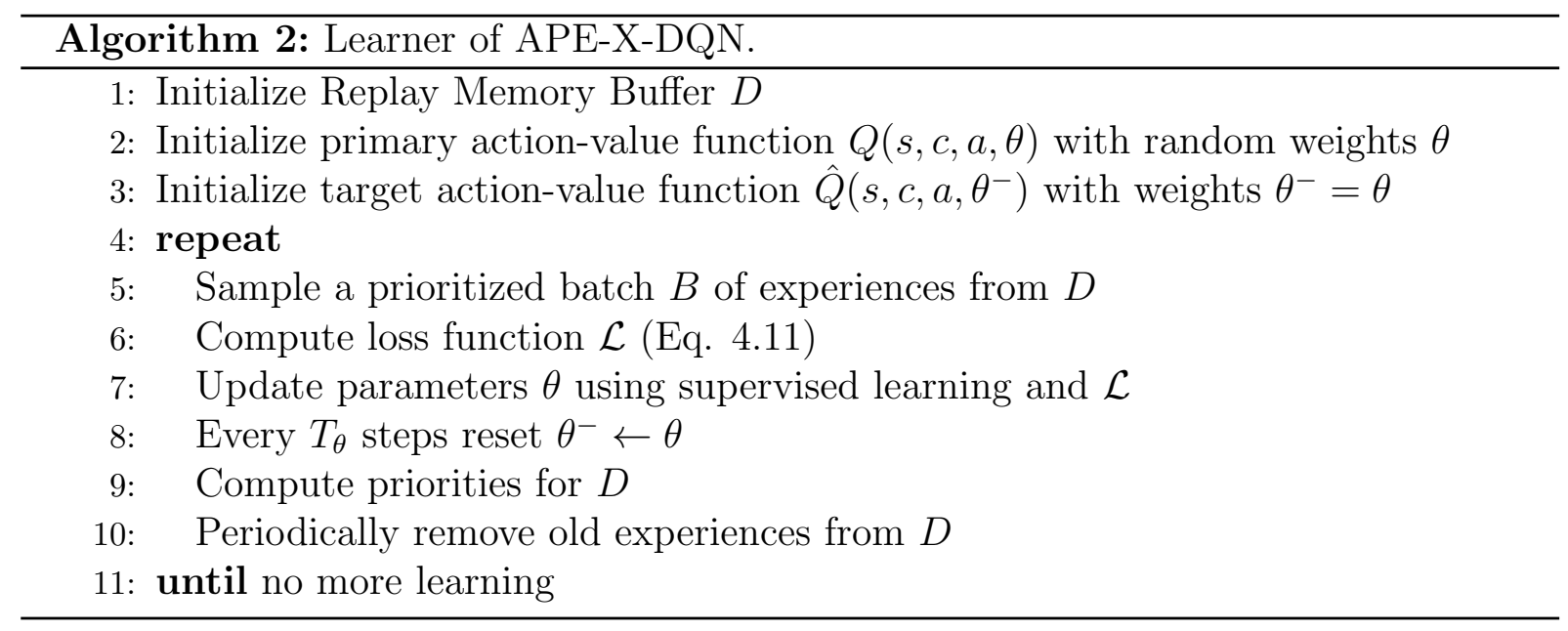

which correspond to the values are then selected. Only sampled experiences have their priority values and IS weights updated by the learner.

A gradient-based algorithm is applied to minimize the loss function with the following learning rule:

$$
\mathcal{L}_{t}(\theta)=1 / 2\left(w_{e}\left(G_{t}-Q\left(s_{t}, c_{t}, a_{t}, \theta\right)\right)\right)^{2},
$$

which is basically the squared weighted TD error with $w_{e}$ as the IS weight for the experience and

$$
G_{t}=r_{t}+\gamma \max _{a} Q\left(s_{t+1}, c_{t+1}, a, \theta^{-}\right)
$$

where $\theta$ represents the primary neural network parameters, and $\theta^{-}$represents the target network parameters. The target network provides the Q-value for TD Error estimation. Its weights $\theta^{-}$are adjusted to the primary network weights $\theta$ every $T_{\theta}$ time steps collected. Using a slow moving copy of the primary network in TD estimation improves learning stability.

Following the original DQN paper suggestion for further stability improvement, the loss function was taken as squared weighted TD error (Equation 4.11) when the error value was between -1 and 1 . The loss was replaced by the absolute TD error $\left(\mid w_{e}\left(G_{t}-\right.\right.$ $\left.\left.Q\left(s_{t}, c_{t}, a_{t}, \theta\right)\right) \mid\right)$ outside this interval. An additional technique commonly applied to stabilization of neural networks in general is the gradient clipping throughout all layers, avoiding abrupt changes in weights.

Each training iteration to the primary network occurs at every $N_{\text {steps-per-iter }}$ experiences collected from actors. Algorithm 2 describes the training process the occur in the learner. 


\subsection{PPO}

The Proximal Policy Optimization (PPO) algorithm was introduced by Schulman et al.(SCHULMAN et al., 2017) and it is an on-policy, PG based algorithm. Although offpolicy VB algorithm might be useful in maritime domain, PPO has gained popularity in the PG category due to its simplicity and stability and is worth exploring for comparison purposes. In this work, PPO was implemented using multiple actors for experience collection, as APE-X-DQN. The difference is that learning occurs in a synchronous fashion - an iteration only occurs after a minimum number of experiences $N_{\exp }$ are collected from actors and they are immediately updated with new policy weights before new experiences are collected. A higher number of experiences can be collected so episodes do not get truncated.

Each learning iteration is performed by applying gradient based optimization in the policy neural network throughout the complete set of experiences collected. The optimization is performed in smaller batches of $B_{P P O}$ of experiences.

In this work formulation, the policy ANN outputs values for all actions and these are used to produce a multinomial probabilistic distribution. Probabilities are formed through a softmax function, as:

$$
\operatorname{softmax}\left(o_{i}\right)=\frac{\exp \left(o_{i}\right)}{\left.\sum_{j} \exp \left(o_{j}\right)\right)},
$$

where $o_{i}$ is the value generated by the output layer for each action $i$. The actions are sampled using that distribution during training. During evaluation, however, the action with highest probability is selected in order to evaluate the trajectories.

Although PPO has some variants, the loss function adopted is:

$$
\mathcal{L}_{t}^{C L I P}(\theta)=\mathbb{E}\left[\min \left(r(\theta)_{t} \hat{A}_{t}, \operatorname{clip}\left(1-\rho_{\text {clip }}, r(\theta)_{t}, 1+\rho_{\text {clip }}\right) \hat{A}_{t}\right)\right]
$$

with:

$$
r(\theta)_{t}=\frac{\pi_{\theta}\left(a_{t} \mid s_{t}\right)}{\pi_{\theta_{\text {old }}}\left(a_{t} \mid s_{t}\right)}
$$

where $\pi_{\theta}\left(a_{t} \mid s_{t}\right)$ and $\pi_{\theta_{\text {old }}}\left(a_{t} \mid s_{t}\right)$ represent the probability of taking action $a_{t}$ at state $s_{t}$ after and before a policy update iteration respectively. $r(\theta)_{t}$ is the ratio of probabilities, representing the importance sampling weight for the specific experience and $\hat{A}_{t}$ is the advantage value estimated for that state action pair. The parameter $\rho_{\text {clip }}$ defines the interval inside which the the loss is clipped. The clipping function is used as an artifact to prevent the policy from receiving large updates and reduces instability during training. 
The advantage value is estimated using a formula proposed by (SCHULMAN et al., 2015), as:

$$
\hat{A}_{t}=\sum_{l=0}^{T}(\gamma \lambda)^{l} \delta_{t+l}^{V},
$$

with $\lambda$ as a factor limiting the horizon of estimation, $T$ is the episode length and

$$
\delta_{t}^{V}=-V\left(s_{t}\right)+r_{t}+V\left(s_{t+1}\right)
$$

where $V\left(s_{t}\right)$ and $V\left(s_{t+1}\right)$ are estimates of value functions. Although the original PPO paper adopts another ANN for value estimation, this work proposes a simplified architecture: The value function estimation uses the same input and hidden layers from policy network and it is given by an extra node placed on the output layer. The complete ANN for PPO policy is illustrated in Figure 15.

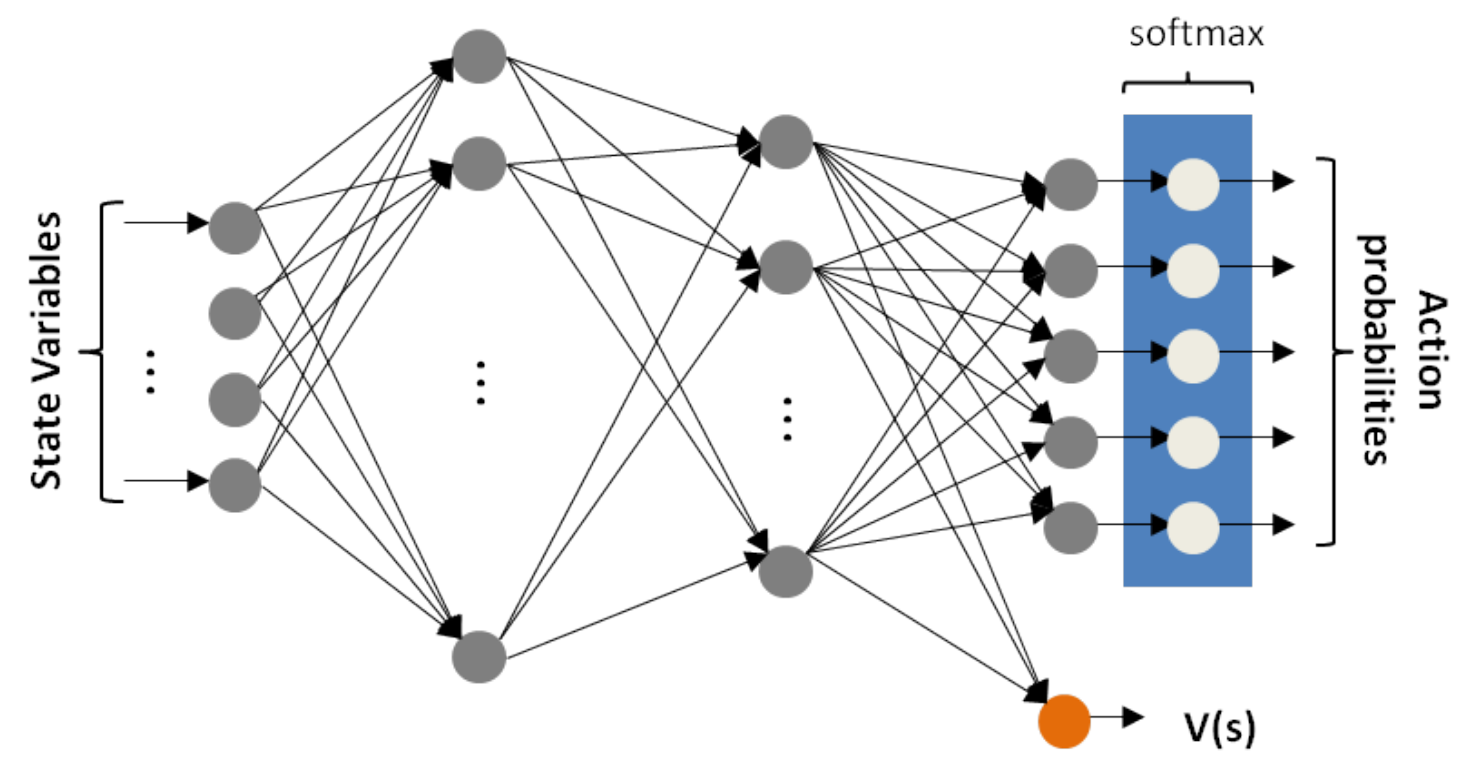

Figure 15: Neural network architecture for PPO policy

Thus, the final loss function must also include a value function error term, as:

$$
\mathcal{L}_{t}^{V F}(\theta)=\mathbb{E}\left[1 / 2\left(V\left(s_{t}\right)-V_{\text {target }}\left(s_{t}\right)\right)^{2}\right]
$$

where the $V_{\text {target }}\left(s_{t}\right)$ is updated for a given experience as $V\left(s_{t}\right)+\hat{A}$.

The final loss function is given as:

$$
\mathcal{L}_{t}^{\text {total }}(\theta)=\mathcal{L}_{t}^{C L I P}(\theta)-\mathcal{L}_{t}^{V F}(\theta)
$$

where $\mathcal{L}_{t}^{V F}$ factor is subtracted because the total loss must be maximized by a gradient 


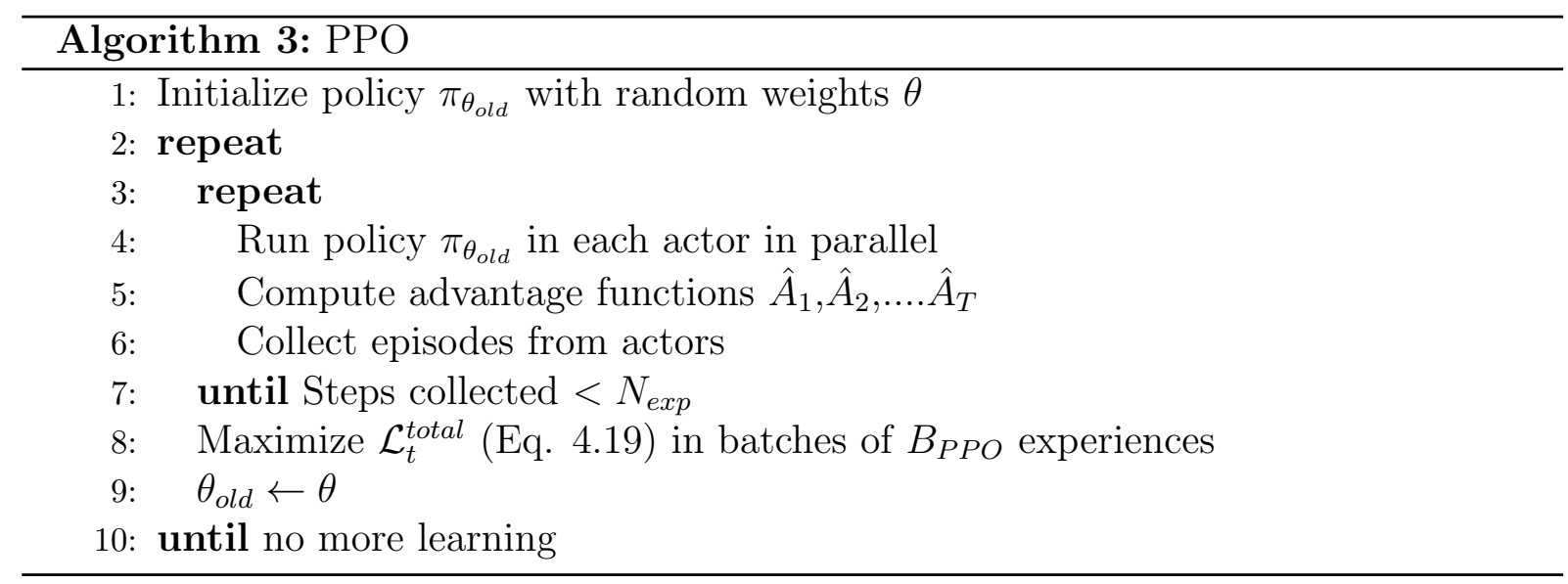

based algorithm in this case. Its weight in the overall loss could be further adjusted, but this work assumed the same weight of $\mathcal{L}_{t}^{C L I P}(\theta)$. Algorithm 3 describes the process. 


\section{RESULTS}

This Chapter shows the outcome of the experiments performed and analyzes them. Section 5.1 depicts the parameters and settings adopted in the algorithms; Section 5.2 presents the vessel model used in the simulations; Section 5.3 presents the scenarios generated for training a generic policy and performance of trials; Both sections 5.4 and 5.5 describe performance of the generic policy in realistic scenarios; Section 5.6 shows improved results when training for a specific channel.

\subsection{Training Settings}

The experiments involved training an agent using both APE-X-DQN and PPO algorithms. As a matter of comparison, all conditions and hyperparamaters that are common to both algorithms were set equally. Because the agent learned navigation under different environmental conditions, massive experience collection was required, so each trial run in $20 \mathrm{CPU}$ cores (1 actor for each) with $2.5 \mathrm{GHz}$ of clock frequency and $128 \mathrm{~GB}$ of RAM memory for each trial. The framework used for implementation was RLLIB (LIANG et al., 2017) with the Python programming language.

For both algorithms, the ANN adopted was a regular Multilayer Perceptron (HAYKIN, 1999) with input of dimension 7 (number of state variables and a context variable). The number of hidden layers used was 2 and the choice was based on a previous effort whose control task solved by RL had a similar complexity (HAFNER; RIEDMILLER, 2011b). The number of perceptrons for hidden layers was chosen as 64 and 32, respectively. The adopted activation function was ReLu (Rectifier Linear Unit) (MAAS; HANNUN; NG, 2013) using ADAM optimizer (KINGMA; BA, 2015). The weights of the perceptrons were updated, among other factors, by the gradient of error weighted by a learning rate, defined as $l_{r}=0.001$.

For APE-X-DQN, the ANN output layer has dimension 5 (number of actions) and the target network was updated with the primary network weights at a rate of $T_{\theta}=8.10^{5}$ 
steps of experience tuples collected. The replay buffer size was set to comprise at most $D=8.10^{5}$ steps. The number of experiences collected until a training iteration occurs was set to $N_{\text {step-per-iter }}=1000$ and the batch size to $B=256$. Prioritization was set with $\alpha_{p r}=0.6$ and $k_{p r}=10^{-6}$. Importance sampling was set with correction $\beta=0.4$. For further training stabilization, all gradients used in ANN optimization were clipped in $[-0.3,0.3]$ interval. The exploration strategy adopted was $\epsilon$-greedy, where each actor was assigned with a different parameter $\epsilon$ that remained constant along training. The value for each actor was defined as:

$$
\epsilon_{i}=0.4^{1+7 \frac{i}{N_{\text {actors }}-1}}
$$

where $i$ represents the actor index and $N_{\text {actors }}$ is the total number of actors: 20 in this case.

For PPO, the ANN output layer has dimension 6 (softmax output for 5 actions and a value function). Each iteration occurred when $N_{\text {exp }}=1000$ were collected. The optimization was performed in batches of size $B_{P P O}=256$. The advantage estimation used $\lambda=1$ and the clipping parameter $\rho_{\text {clip }}=0.3$

The discount factor was set to $\gamma=0.99$. The numerical integration step in the training process is $\Delta T_{\text {train }}=2 s$. This is a trade-off between computational performance and integration accuracy of the mathematical model differential equations. However, the trained policies were evaluated in time-domain simulations using $\Delta T_{\text {eval }}=0.1 \mathrm{~s}$, as means of assuring that the coarseness of $\Delta T_{\text {train }}$ does not generate unsatisfactory policies when the fast-time simulations are executed with smaller integration step.

The distance between way-points $L_{\text {segment }}$ considers the trade-off mentioned in Section 4.2 , so that the desired path segment does not intersect margins considering the lowest radius of the bends $r$ and the minimum channel width $w$ compatible with real-world channels. The adopted values are compliant with the Harbour Approach Channels Design Guidelines (MCBRIDE et al., 2014). For the navigation speed of $4 \mathrm{~m} / \mathrm{s}$, under moderate crosswind and cross current velocities, $w=4$ Beam $\approx 190 \mathrm{~m}$. The minimum value for bend radius is $r=5 L_{o a} \approx 1400 m$, where $L_{o a}$ is the vessel length. Considering this scenario as the bottleneck, $L_{\text {segment }}=650 \mathrm{~m}$. 


\subsection{Vessel Model and Training Scenario}

The vessel type used was a full loaded Suezmax DP tanker (160,000DWT). Although this ship has bow and stern thrusters, the navigation along the access channel was carried out only with the machine (propeller) and rudder. The ship's characteristics in shallow and deep waters are shown in the Figure 16.

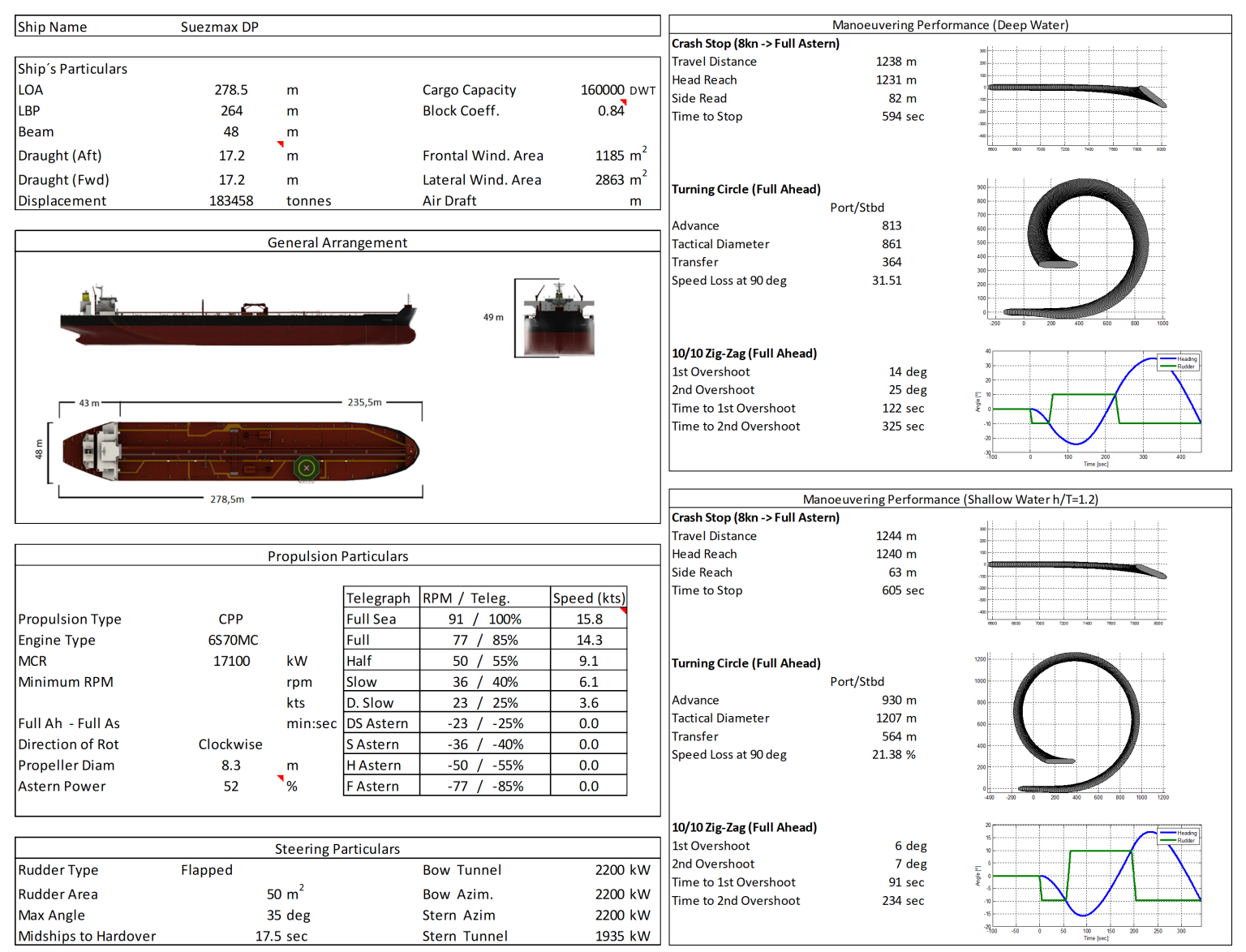

Figure 16: Suezmax DP tanker: (left) main characteristics; (right) maneuvering properties.

\subsection{Generic Policy Training}

As a way to explore the generalization capacity of the proposed representation, the policy was trained in scenarios that represent typical topologies and environmental conditions so that it could be further evaluated in realistic channels. A fictitious channel, illustrated in Figure 17 was generated with segments that approximate curvatures with radius of $2100 \mathrm{~m}, 1600 \mathrm{~m}$ and $1100 \mathrm{~m}$ respectively. The channel breadth is $190 \mathrm{~m}$ in straight portions and $240 \mathrm{~m}$ in curved ones. 


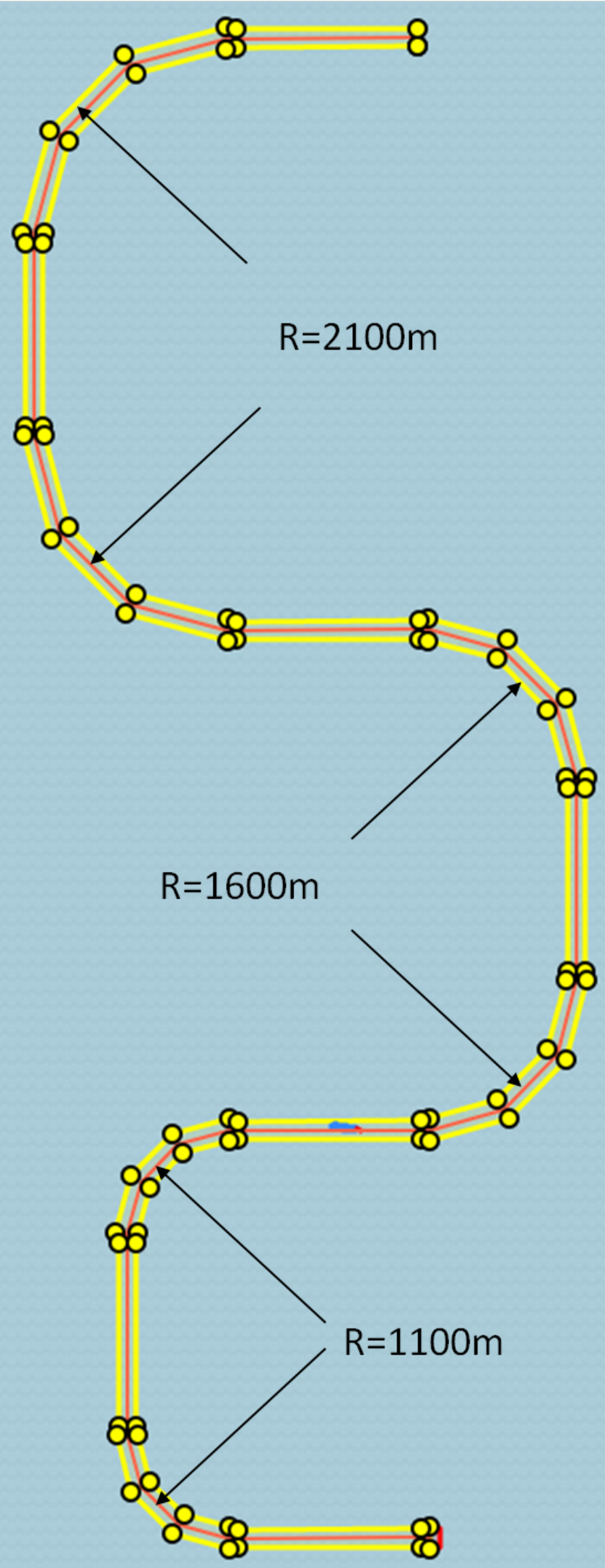

Figure 17: Channel topology generated for training 
it is exposed to forces in a variety of magnitude and directions that can occur in realistic scenarios. The training scenarios were generated according to the cartesian product $m a g_{\text {wind }} \times d i r_{\text {wind }} \times \operatorname{mag}_{\text {cur }} \times d i r_{c u r}$, where the sets represent wind velocity, wind direction, current velocity and current direction respectively. Velocity is given in knots $(1 \mathrm{kn} \approx 0.51$ $\mathrm{m} / \mathrm{s}$ ) and direction is given degrees considering 0 at north and clockwise oriented. The training was performed using $\operatorname{mag}_{\text {wind }}=\{0.0,12.0,24.0\} \mathrm{kn}, \operatorname{dir}_{\text {wind }}=\{0,90,180,270\}^{\circ}$, $\operatorname{mag}_{\text {cur }}=\{0.0,0.4,0.8,1.0\} \mathrm{kn}$ and $d i r_{c u r}=\{0,90,180,270\}^{\circ}$, which means that experiences were collected in the fictitious channel under 186 different environmental scenarios.

Waves were not included in these experiments as their simulation drastically increase computational time, but preliminary experiments developed in the early stage of this work demonstrated that policies can learn to navigate with presence of waves even without information in state space, as published in (AMENDOLA et al., 2019).

Three different trials were run with APE-X-DQN and PPO. A typical stop criterion adopted in $\mathrm{RL}$ is the convergence of mean accumulated reward per episode (or mean return).

The metrics are displayed in terms of experience sets (or steps) collected. That makes analysis independent of computational resources and also indicates the sample efficiency of training.

Figure 18 displays the average accumulated reward as training progresses. It grows in the beginning and it stabilizes in a near-optimal situation. Marginal oscillations are common as episodes run over different portions of the channel. Although such oscillations occur for both algorithms, that was slightly lower for PPO, which also displayed a slower convergence. 


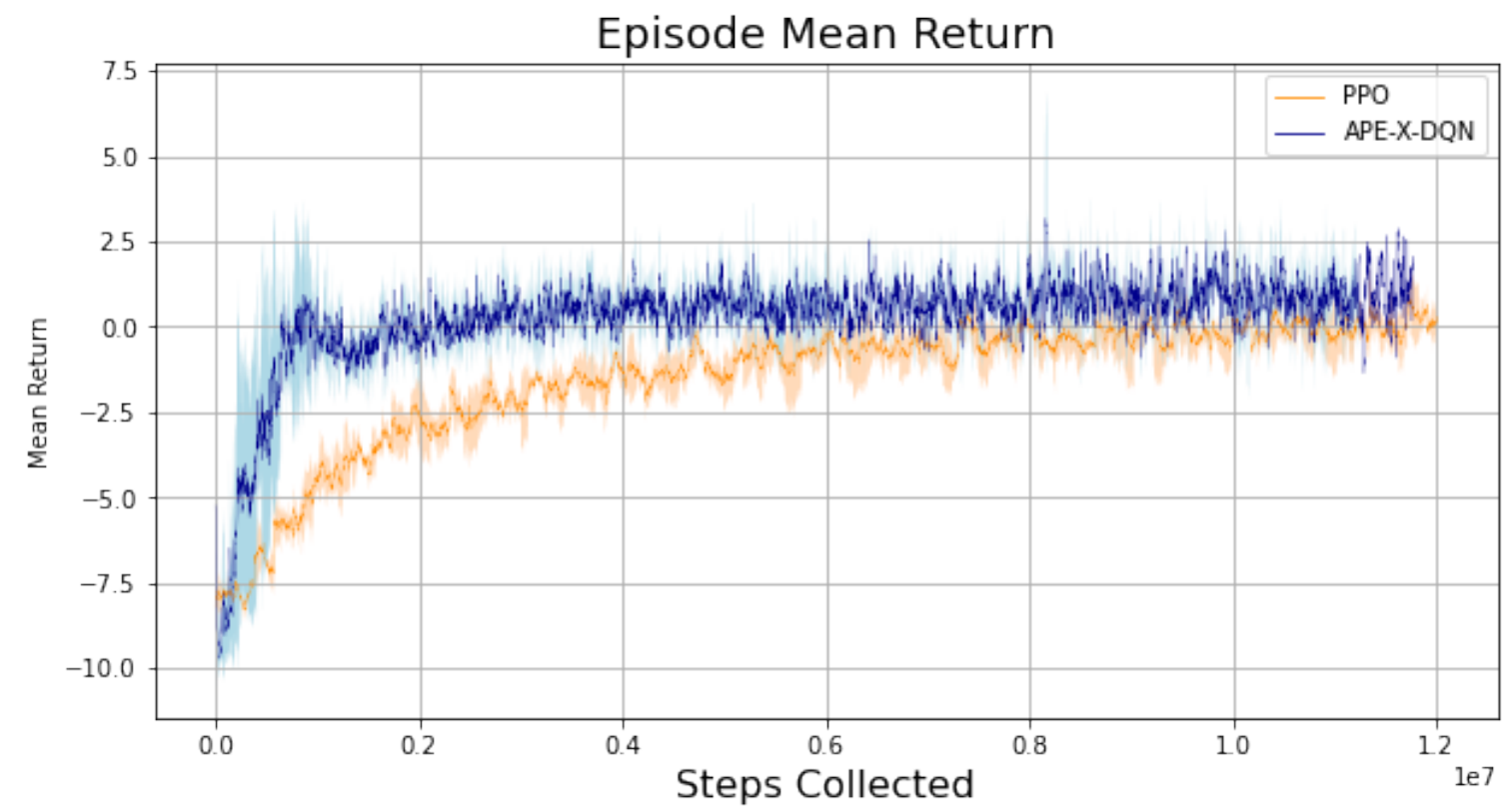

Figure 18: Average accumulated reward per episode (moving average from 100 episodes). Mean from 3 learning trials in darker orange and darker blue; Standard deviation in shaded lighter orange and lighter blue.

Figure 19 also conveys the training improvement as the average episode length grows, meaning that the vessels are navigating longer distances without collision.

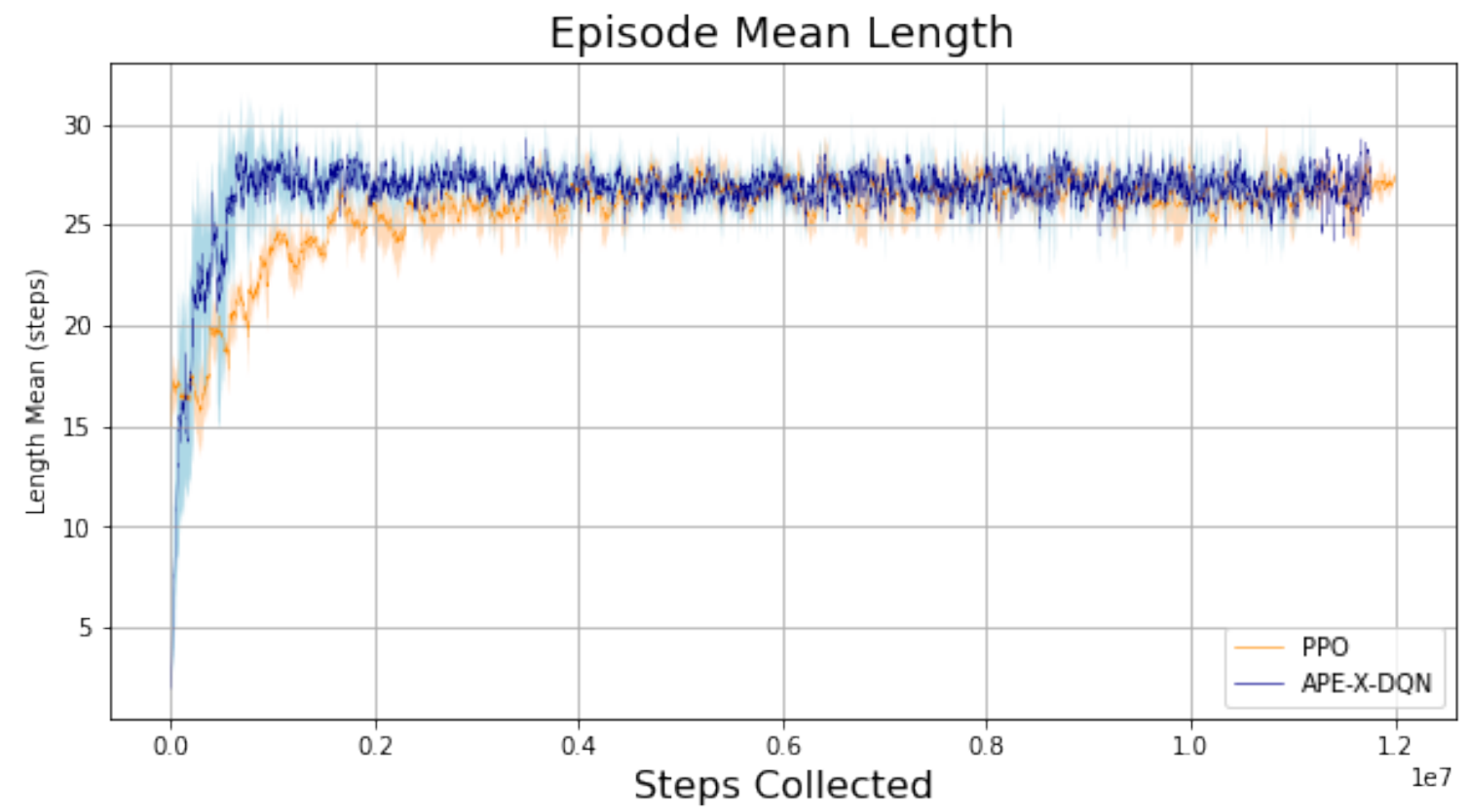

Figure 19: Average episode length (moving average from 100 episodes). Mean from 3 learning trials in darker orange and darker blue; Standard deviation in shaded lighter orange and lighter blue.

As mean return accounts only the episode comprised between two line segments, a 
periodic evaluation is necessary considering a complete channel. At each 10 training iterations, the policy was evaluated with exploration suspended in the full extent of Porto Sudeste with flood current of $5 \mathrm{kn}$ (See Section 5.4). Figure 20 shows the maximum fraction of the channel that the vessel was able to navigate as the policy was improved. The value shows high variance among trials as small differences in policy can make the vessel collide in early curves. The training instability is more evident for APE-X-DQN. PPO training asymptotically convergences slower than APE-X-DQN, but it presents a more stable policy after $6.10^{6}$ steps. In order to obtain a stability margin, the policy was trained until $9.10^{6}$ so further analysis was conducted in realistic channels.

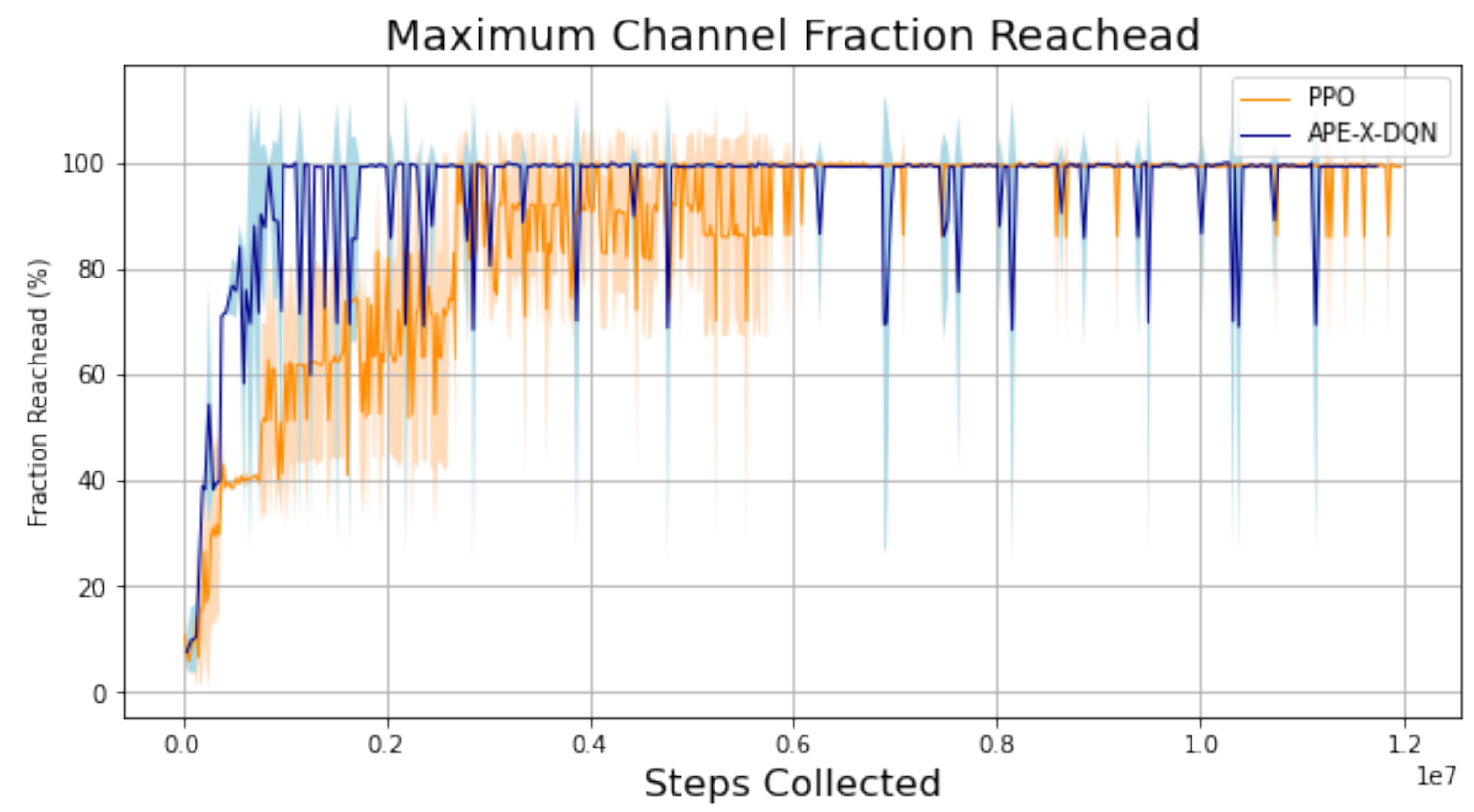

Figure 20: Fraction of channel reached by periodic evaluation of policy. Mean from 3 learning trials in darker orange and darker blue; Standard deviation in shaded lighter orange and lighter blue.

Although APE-X-DQN has presented a faster convergence to a successful policy according to the evaluation, it presented more instability training process. Obtaining desired policies from unstable training runs are more difficult as performance varies abruptly among iterations. That demands more frequent evaluations in a complete channel during trials and can hence undermine algorithm efficiency.

\subsection{Evaluation in Porto Sudeste Channel}

The proposed method was analyzed in an environment simulating the Porto Sudeste Access Channel. It is located in Sepetiba Bay, State of Rio de Janeiro, in the Southeast 
region of Brazil (Figure 21).

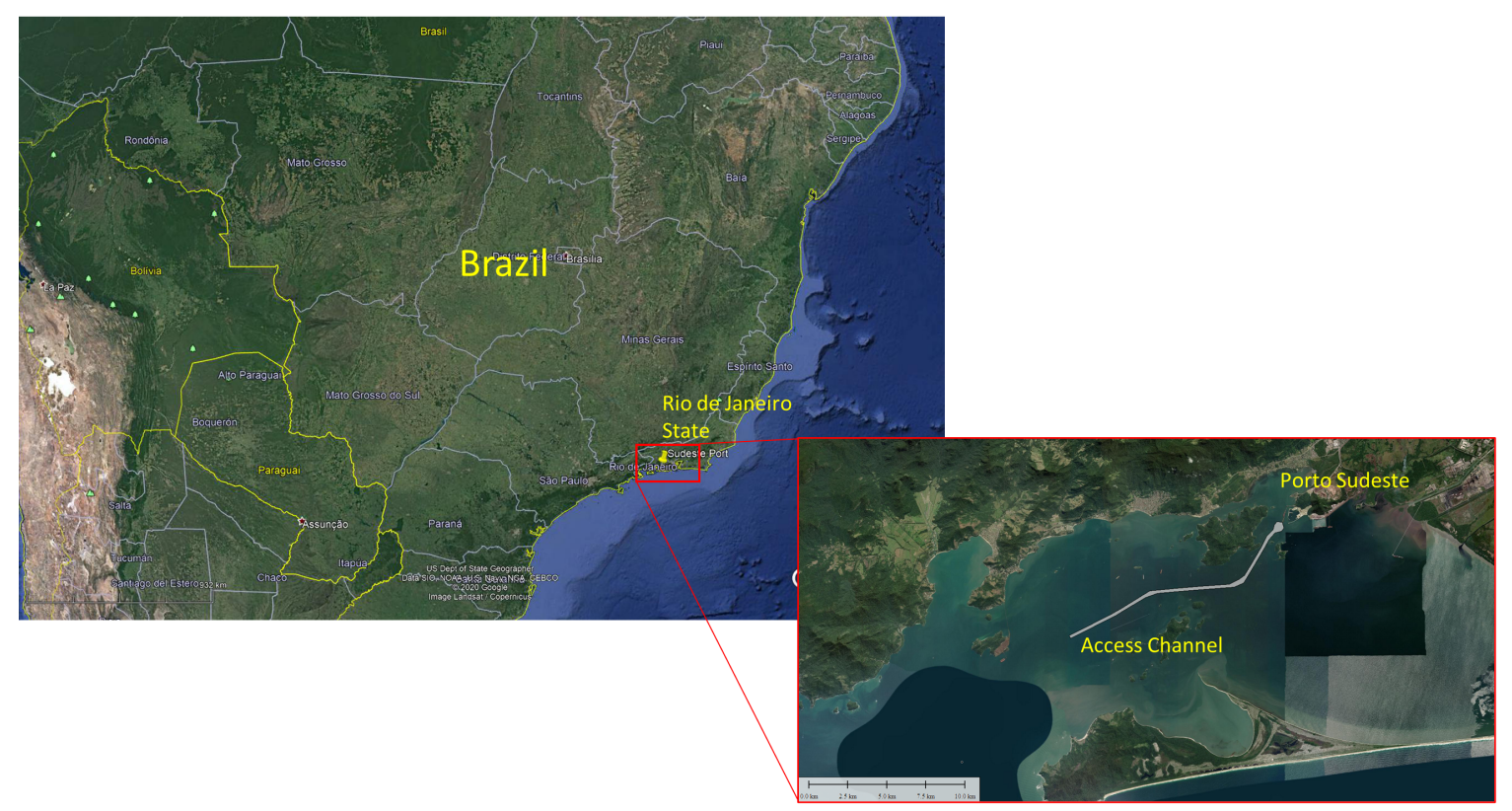

Figure 21: Location of the Porto Sudeste Access Channel.

The simulated section of the access channel is approximately $16 \mathrm{~km}$ (8.6 nautical miles) long, in three straight parts with a $58^{\circ}, 85^{\circ}$ and $30^{\circ}$ heading, respectively. The minimum depth of the channel is $19 \mathrm{~m}$ and the minimum width is $206 \mathrm{~m}$. There is a bend to access the final stretch of the channel, with a radius of $1600 \mathrm{~m}$ (Figure 22).

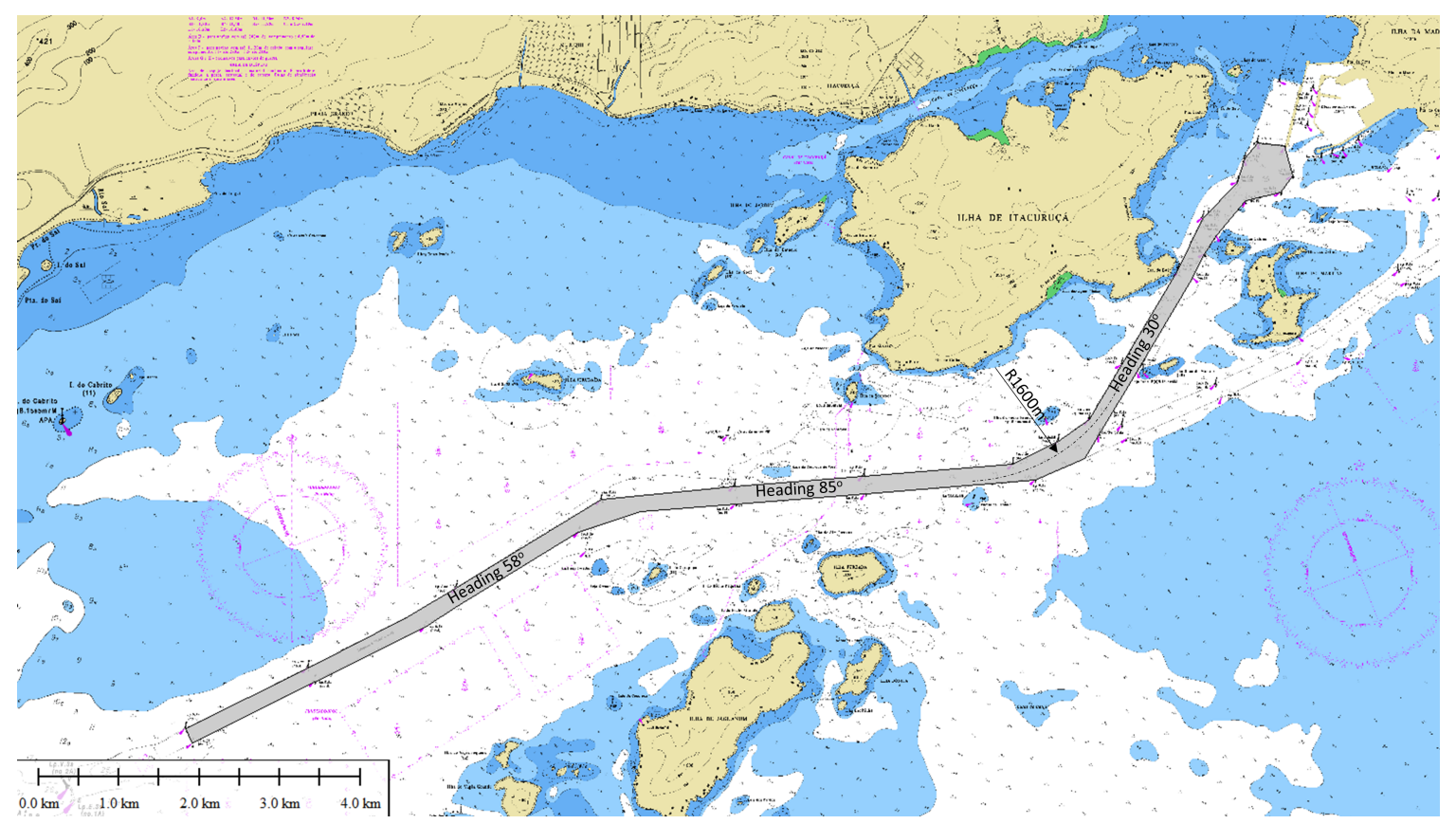

Figure 22: Porto Sudeste Access Channel over the Nautical Chart.

Both Flood and Ebb current conditions were considered, with speed (measured at 
the main curve) from 0.4 to $1.0 \mathrm{kn}$. The $20 \mathrm{kn} \mathrm{W}$ and $\mathrm{N}$ wind were associated with the flood and ebb scenarios, respectively. Figure 23 shows the current in the navigation channel obtained from a validated hydrodynamic model. Table 5 shows the simulated environment.

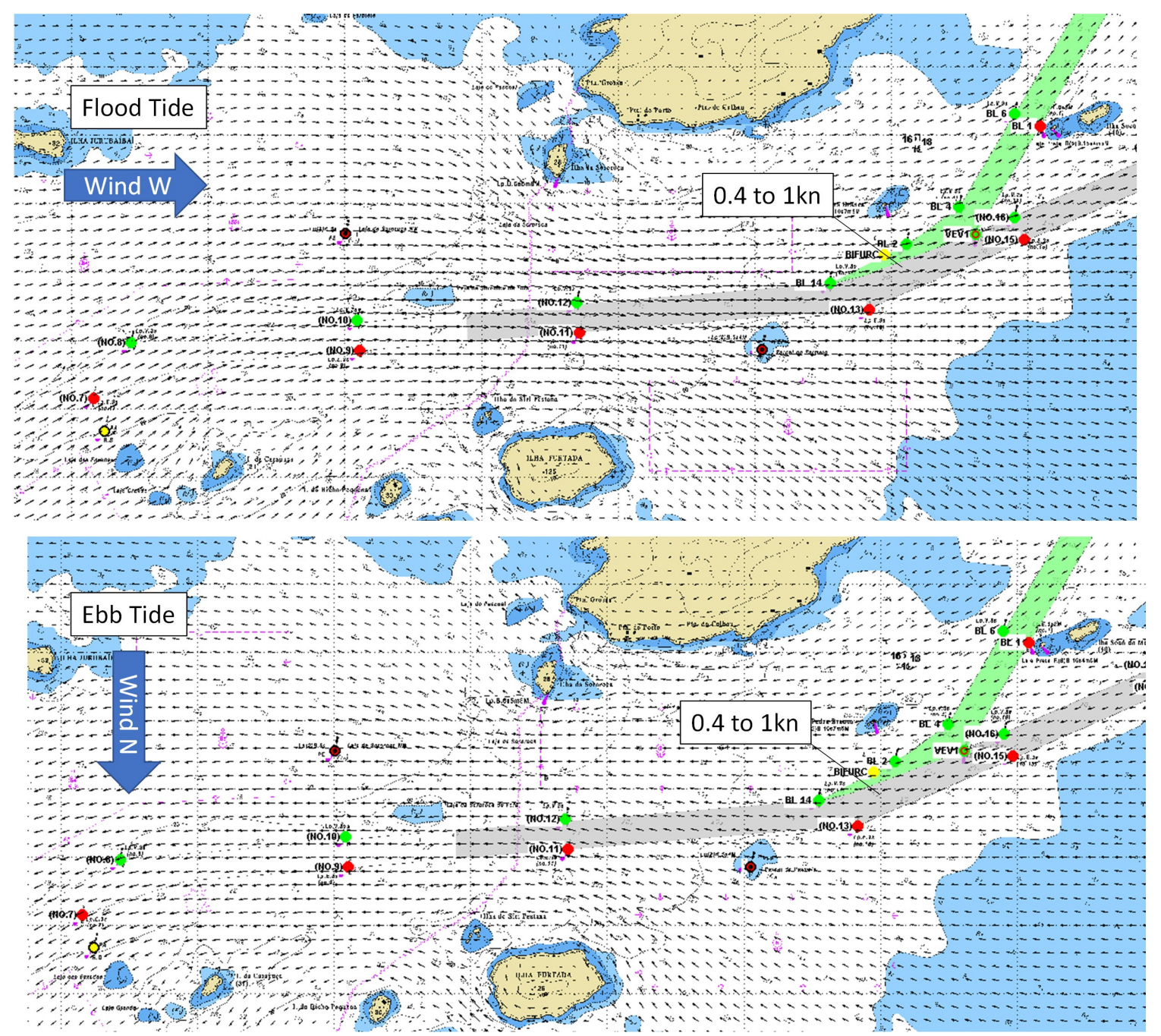

Figure 23: Environmental conditions - Current Vectors and Wind Direction: (up) Flood tide scenario; (down) Ebb tide scenario.

Table 5: Environmental scenarios evaluated in Porto Sudeste.

\begin{tabular}{|l|l|l|}
\hline Scenario & Current & Wind \\
\hline E04 $(0.4 \mathrm{kn})$ to & Direction: Ebb & Direction: N \\
E10 $(1.0 \mathrm{kn})$ & Speed: 0.4kn; 0.5kn; 0.8kn; 1.0kn & Speed: 20kn \\
\hline F04 $(0.4 \mathrm{kn})$ to & Direction: Flood & Direction: W \\
F10 $(1.0 \mathrm{kn})$ & Speed: 0.4kn; 0.5kn; 0.8kn; 1.0kn & Speed: 20kn \\
\hline
\end{tabular}

Figure 24 illustrates trajectories obtained by a policy trained with APE-X-DQN for 
conditions E04, E05, E08 and E10. The vessel was able to maneuver in both curves, even under the effect of environmental forces. Note that the policy can anticipate the change in the orientation of desired paths segments so as to avoid collisions in curves due to large overshooting.

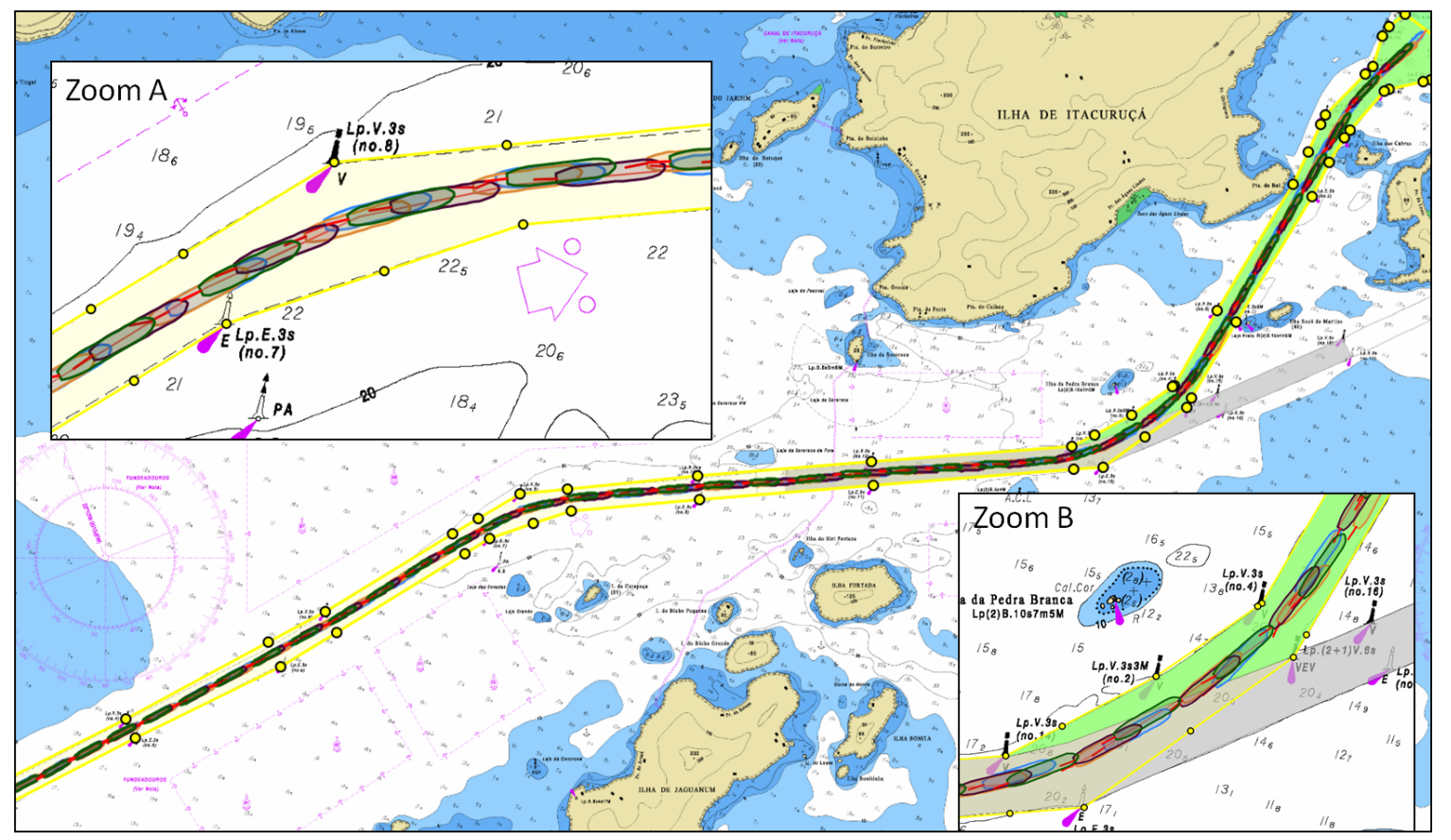

Figure 24: Trajectories for policy trained with APE-X-DQN. Blue ship: Scenario E04; Orange ship: Scenario E05; Purple ship: E08, Green ship: E10.

A more thorough analysis was performed for scenario F05. The curves obtained for PPO and APE-X-DQN were plotted in different charts because small differences in navigation velocity cause policies to experience channel parts with a small offset in time and might make analysis difficult.

The vessel's heading, course over ground $(\operatorname{cog})$, and the channel centerline orientation for F05 using APE-X-DQN are shown in Figure 25. The heading and $\operatorname{cog}$ are ahead of the channel orientation angle, showing the ability to anticipate the curve, as a result of the state-space model that contains information of the curvature ahead and distance to the orientation change. The difference between $\operatorname{cog}$ and heading is the drift angle, required for compensating the perpendicular environmental forces. The value is less than $5^{\circ}$ in the present case and is also employed by human pilots as a measurement of how difficult the course keeping is. 


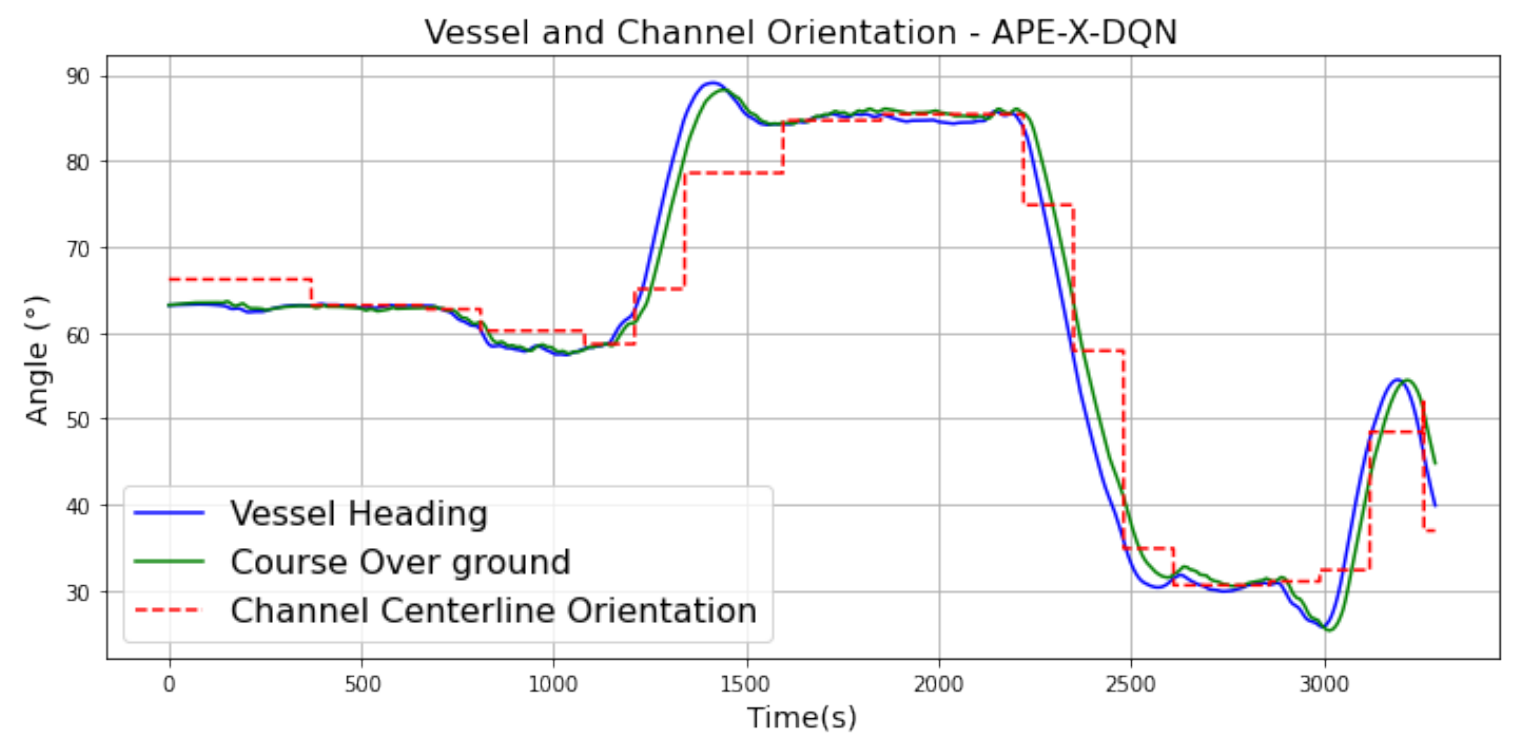

Figure 25: Vessel's heading and course over ground in Scenario F05 for APE-X-DQN

The same chart is shown for PPO algorithm, demonstrating the same qualitative behavior as observed for APE-X-DQN, with small differences in values. Although policies trained for the same MDP in both cases, they are not exactly the optimal solution and thus, it is expected that they present differences in behavior.

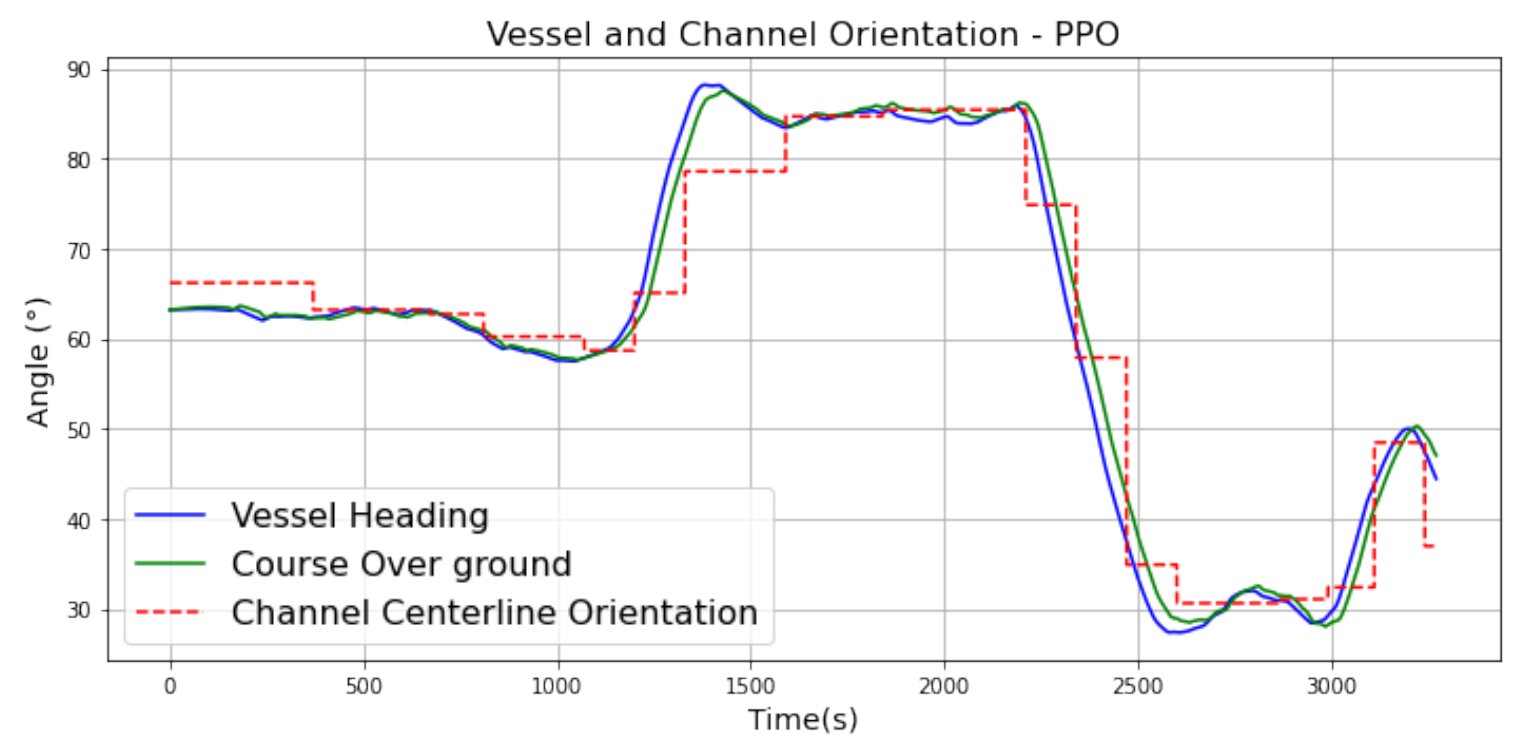

Figure 26: Vessel's heading and course over ground in Scenario F05 for PPO

The actions (rudder angles) are shown in Figure 27, for a 500s time-window . The agent applies minimum positive rudder angle to keep the required drift angle for counteracting the environmental forces in straight segments $(t<1000 s)$. During the curve, there is a higher rudder action necessary to control the rate-of-turn. The frequency of rudder 
commands is similar to a human-controlled maneuver. Frequent angle inversions occur to avoid high rate-of-turn values and further destabilization.

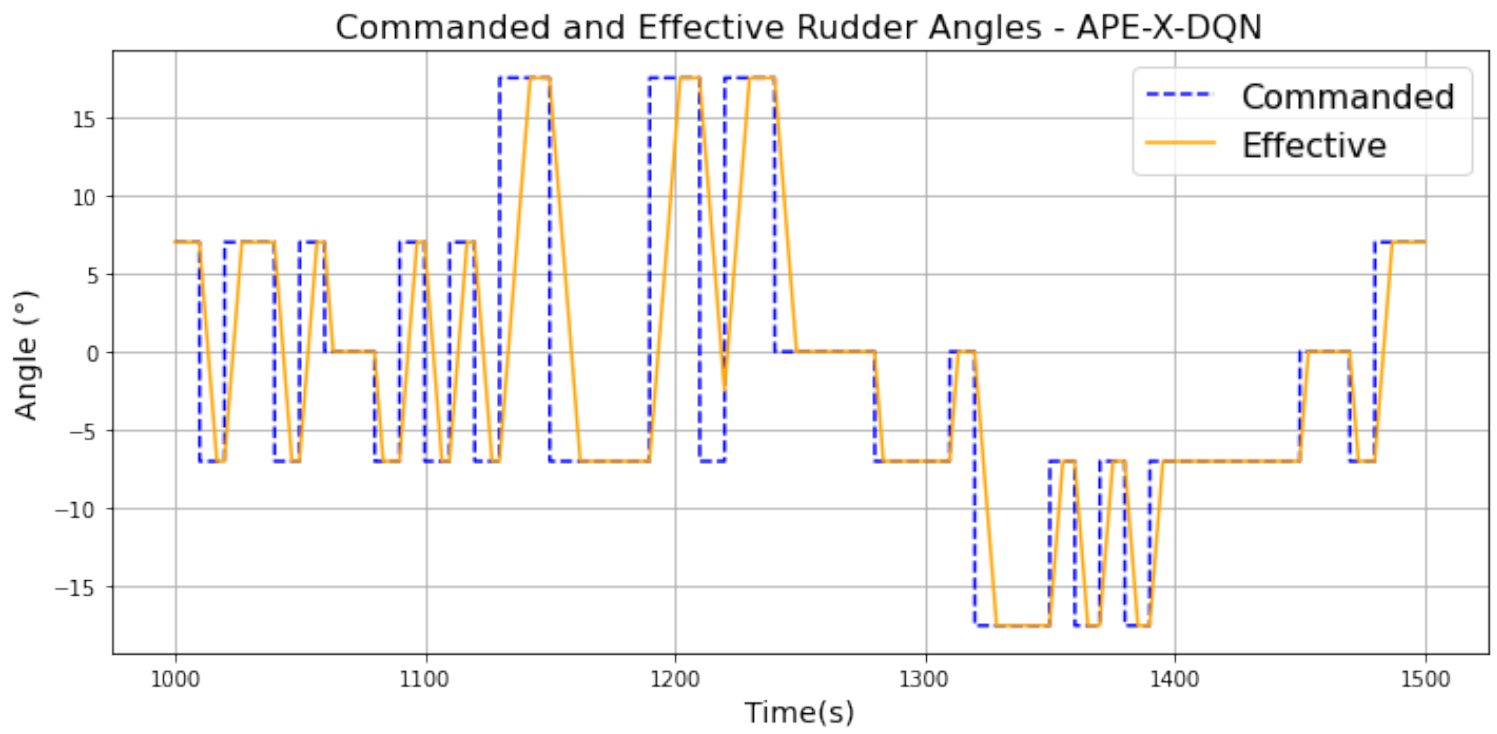

Figure 27: Rudder angles commanded by the agent and its effective value in Scenario F05 for APE-X-DQN

The actions taken with PPO (Figure 28) reveal a different strategy. Although the policy managed to navigate the channel, the angle inversions are much less frequent in the straight segment. Higher angles were also required for the curve.

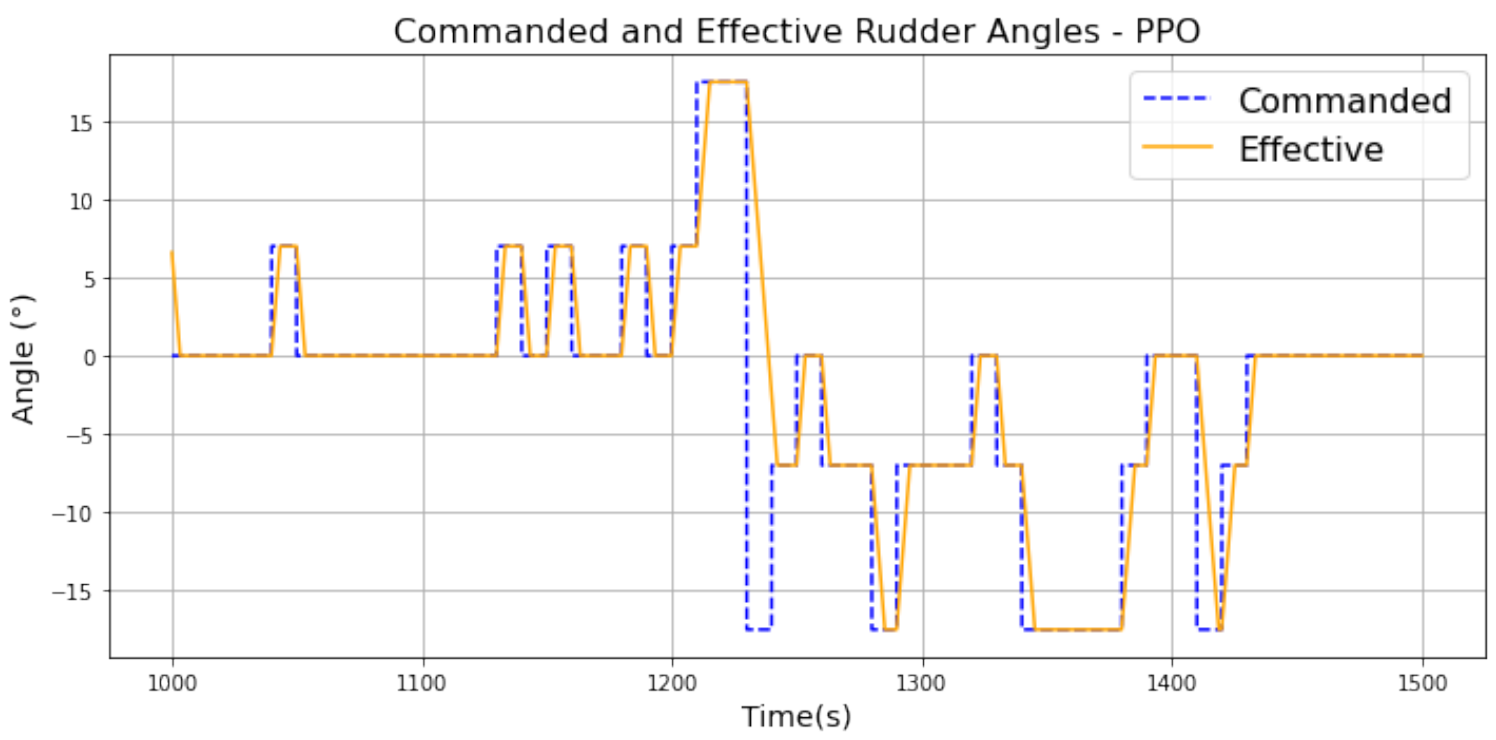

Figure 28: Rudder angles commanded by the agent and its effective value in Scenario F05 for $\mathrm{PPO}$

Although the maneuver is executed with a fixed propeller command, the speed over ground varies from $3.7 \mathrm{~m} / \mathrm{s}$ to $5.3 \mathrm{~m} / \mathrm{s}$, due to the variation of the longitudinal environmen- 
tal forces and the speed loss after curves (Figures 29 and 30). This variation is sufficiently small such that the RL agent (that does not take the speed as a state variable) could accurately control the vessel's maneuver. The experiences acquired during training enabled the agent to learn how to generalize the vessel's behavior in this speed interval. The vessel stops accelerating in the curves because the higher rudder angles cause an increase in hydrodynamic resistance forces.

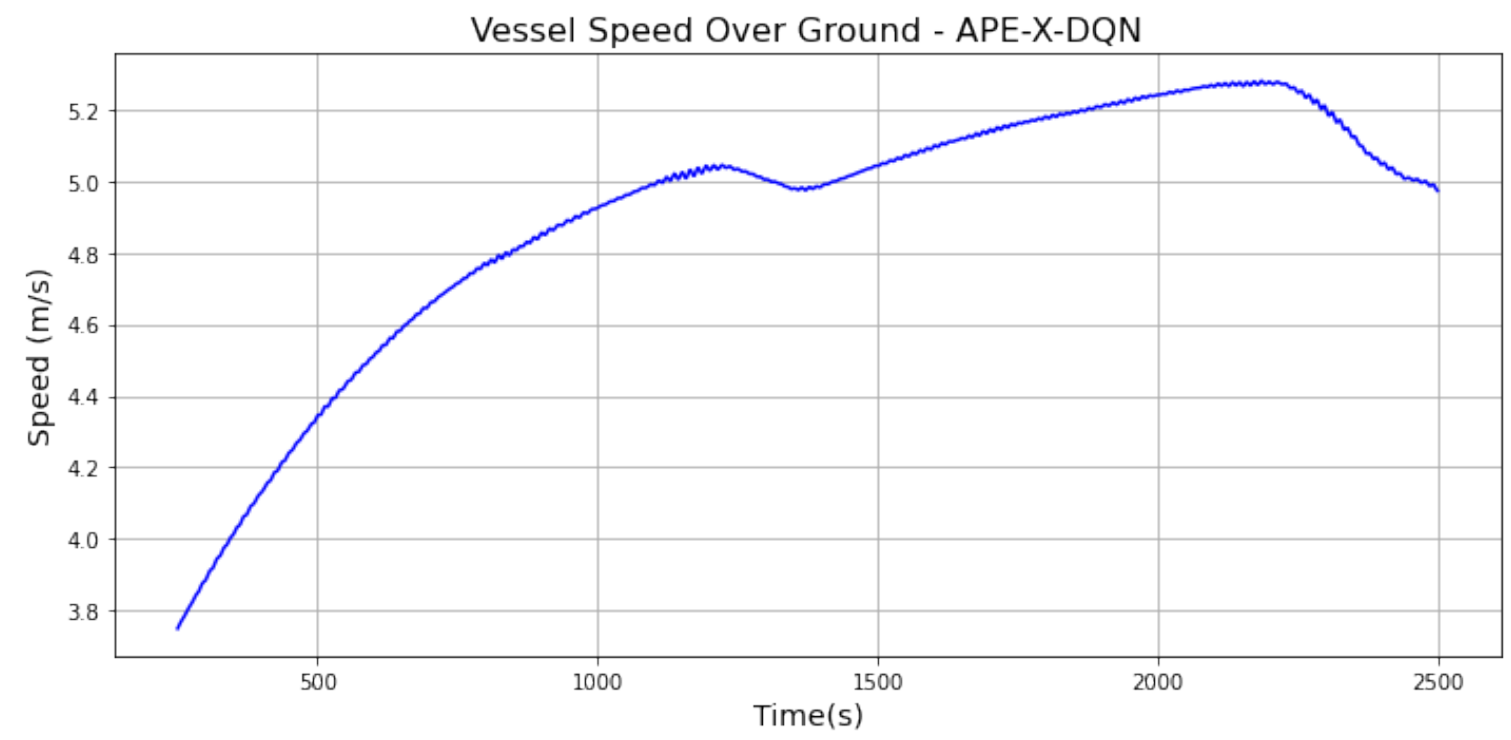

Figure 29: Speed over ground along the navigation in Scenario F05 using APE-X-DQN.

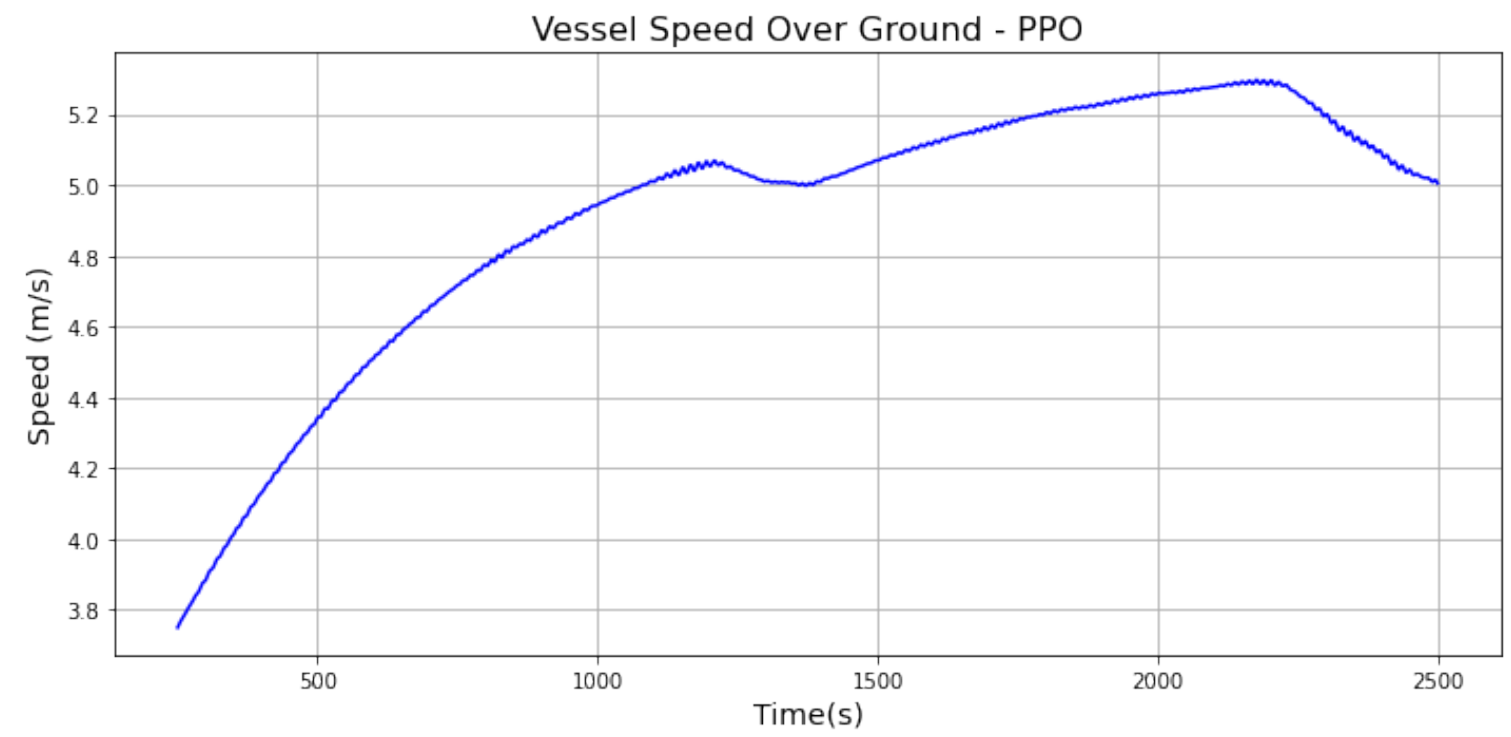

Figure 30: Speed over ground along the navigation in Scenario F05 using PPO.

The distances to margins for all Porto Sudeste evaluated scenarios are depicted in Figure 31 for APE-X-DQN and in Figure 32 for PPO. All trajectories succeed in reaching 
the end of the channel. The vessel was able to navigate at safe distances from margins, getting closer to them when encountering curves, specially the one for the interval between $t=1250 \mathrm{~s}$ and $t=1500 \mathrm{~s}$. The small differences in velocity for different scenarios are evident with the offset among valleys in the chart. Minimum distances, however, are acceptable for most scenarios, as usual acceptance criteria adopted by pilots are distances larger than 0.5 Beam $\approx 23 m$. The only trajectory that violated it was for F10 using APE$\mathrm{X}$-DQN. In this case, the current direction tends to push the vessel towards left margin in the curve and the wind coming from north causes a momentum that rotates the vessel bow towards the same margin. This condition makes this curve particularly critical.

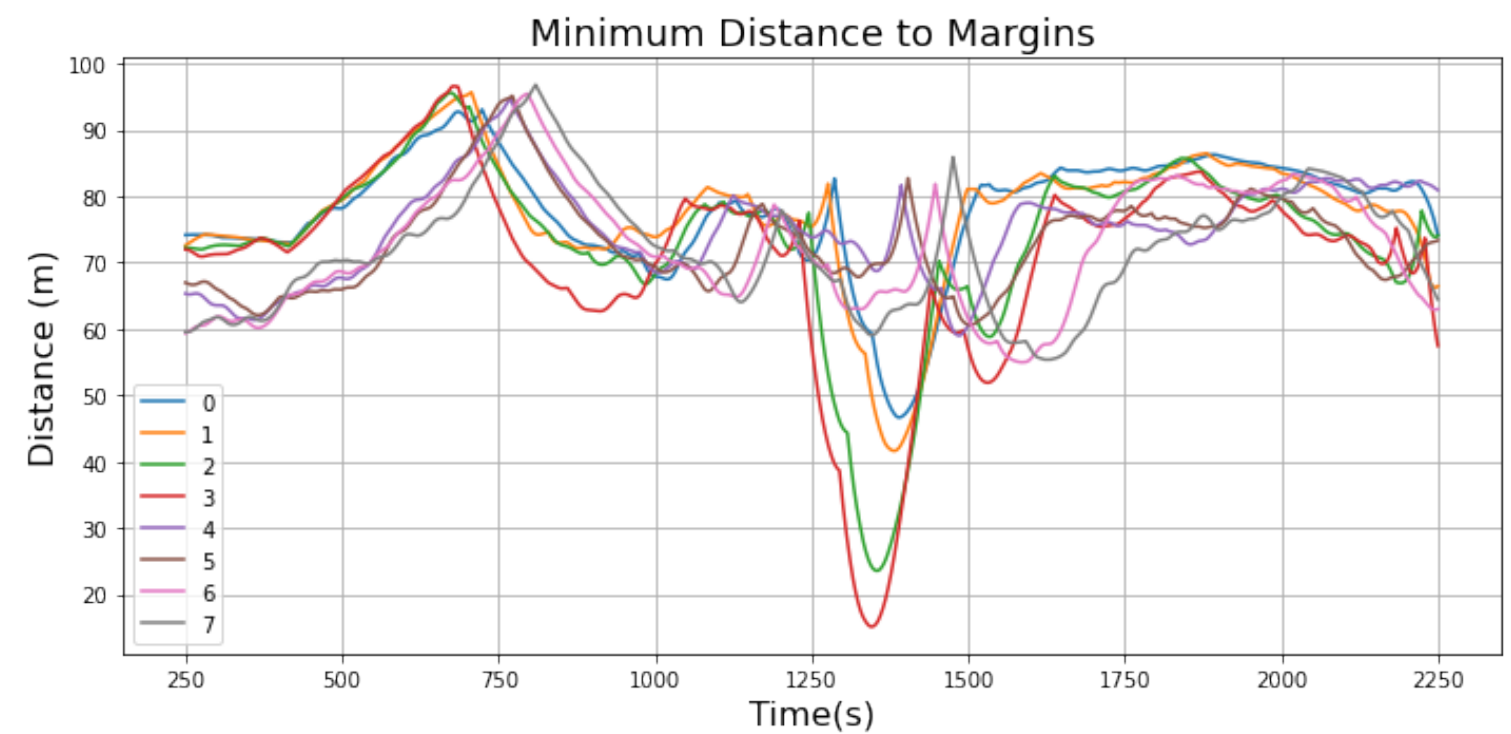

Figure 31: Distance to margins at Porto Sudeste for scenarios using APE-X-DQN. 0-F04, 1-F05, 2-F08, 3-F10, 4-E04, 5-E05, 6-E08, 7-E10 


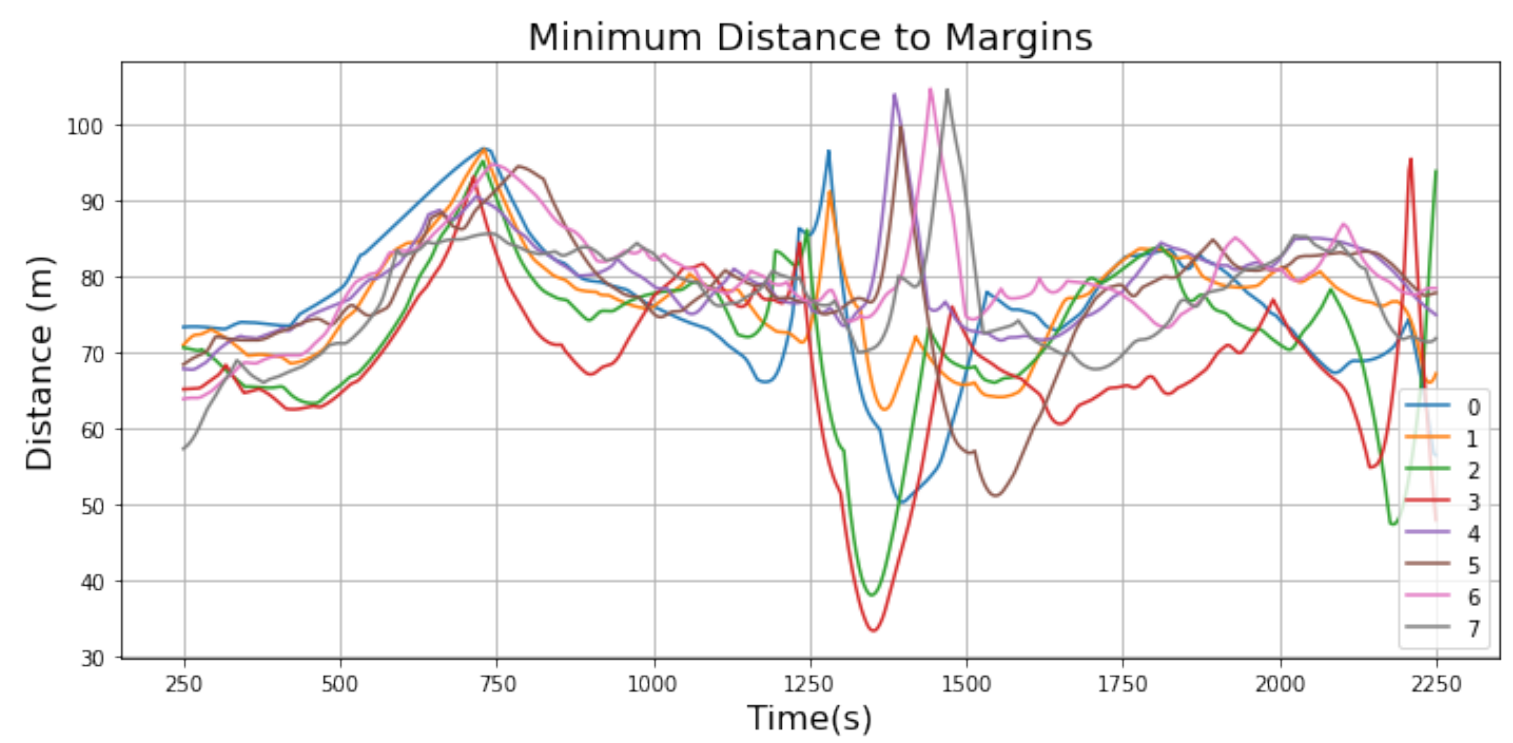

Figure 32: Distance to margins at Porto Sudeste for scenarios using PPO. 0-F04, 1-F05, 2-F08, 3-F10, 4-E04, 5-E05, 6-E08, 7-E10

The policy obtained was able to keep track of the desired path segments at the same time that environmental forces were compensated; that was achieved with rudder commands that closely resemble human piloting. As expected, the presence of a context variable and the distance to way-point provided to the policy improved the ability to anticipate curves. Extrapolation of the policy trained to conditions of Porto Sudeste was achieved because experiences under different scenarios were processed during training. That strategy prevented the neural network from overfitting to one environmental scenario. Better trajectories, however, can be obtained by continuing the policy training process using the specific channel and the realistic environmental scenarios.

\subsection{Evaluation in Suape Channel}

The policy trained in the fictitious channel was also evaluated in the scenario representing Suape Channel, located in northeast of Brazil (Figure 33). 


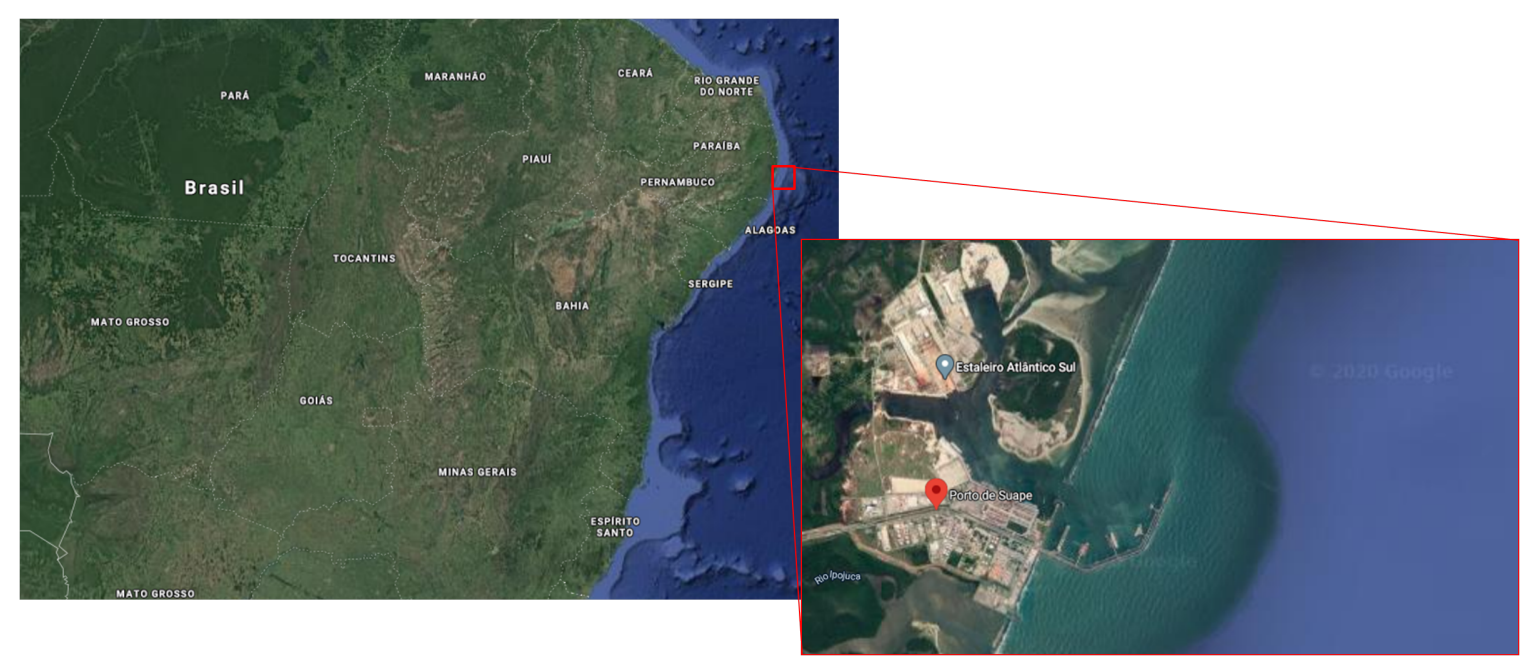

Figure 33: Location of Suape Access Channel

Differently from Porto Sudeste, the topology contains only one long bend, as illustrated in Figure 34. It is composed by two straight segments: The first has width $240 \mathrm{~m}$ and heading of $-13.5^{\circ}$. The second has width $210 \mathrm{~m}$ and heading of $-104^{\circ}$.

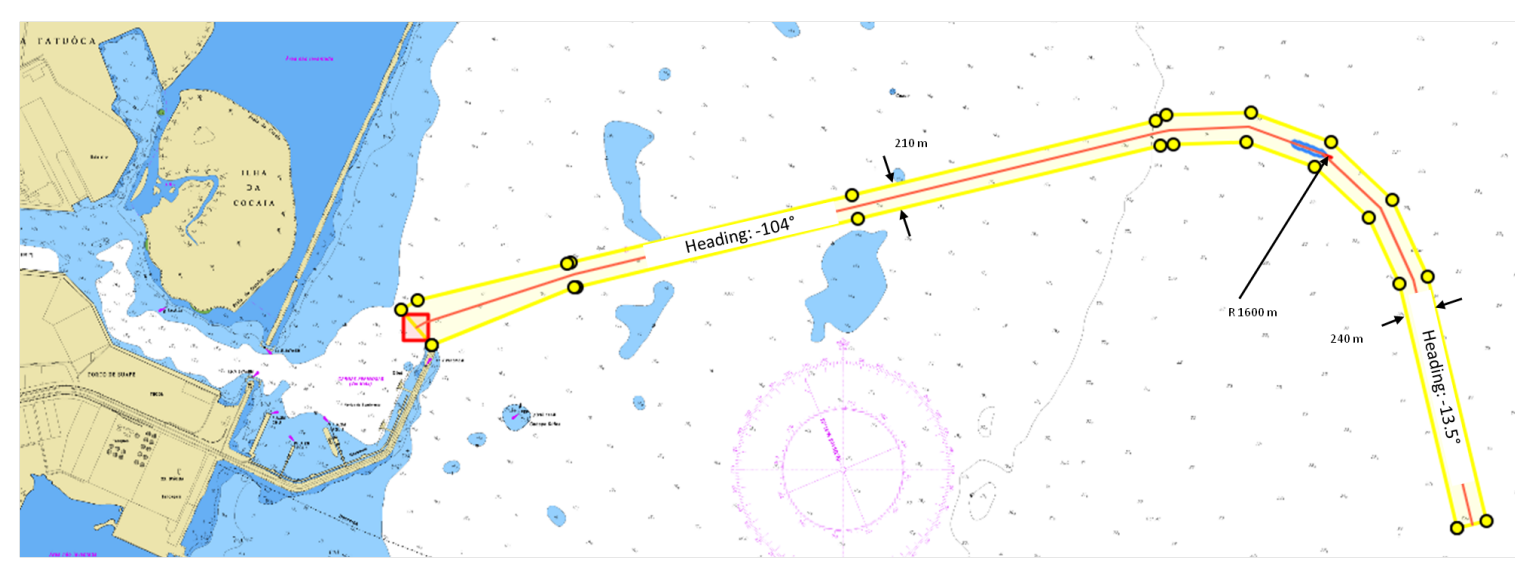

Figure 34: Suape Access Channel over the Nautical Chart

Table 6 lists all environmental scenarios considered for evaluation at Suape channel. Those are realistic conditions deemed representative for the area. Those are further illustrated in Figure 35 
Table 6: Environmental scenarios evaluated in Suape.

\begin{tabular}{|l|l|l|}
\hline Scenario & Current & Wind \\
\hline N04W20 & $\begin{array}{l}\text { Direction: North } \\
\text { Speed: 0.4kn }\end{array}$ & $\begin{array}{l}\text { Direction: } 225^{\circ} \\
\text { Speed: 20kn }\end{array}$ \\
\hline N08W20 & $\begin{array}{l}\text { Direction: North } \\
\text { Speed: 0.8kn }\end{array}$ & $\begin{array}{l}\text { Direction: } 315^{\circ} \\
\text { Speed: 20kn }\end{array}$ \\
\hline N08W24 & $\begin{array}{l}\text { Direction: North } \\
\text { Speed: 0.8kn }\end{array}$ & $\begin{array}{l}\text { Direction: } 225^{\circ} \\
\text { Speed: 24kn }\end{array}$ \\
\hline N08W20-330 & $\begin{array}{l}\text { Direction: North } \\
\text { Speed: 0.8kn }\end{array}$ & $\begin{array}{l}\text { Direction: } 330^{\circ} \\
\text { Speed: } 14 \mathrm{kn}\end{array}$ \\
\hline
\end{tabular}

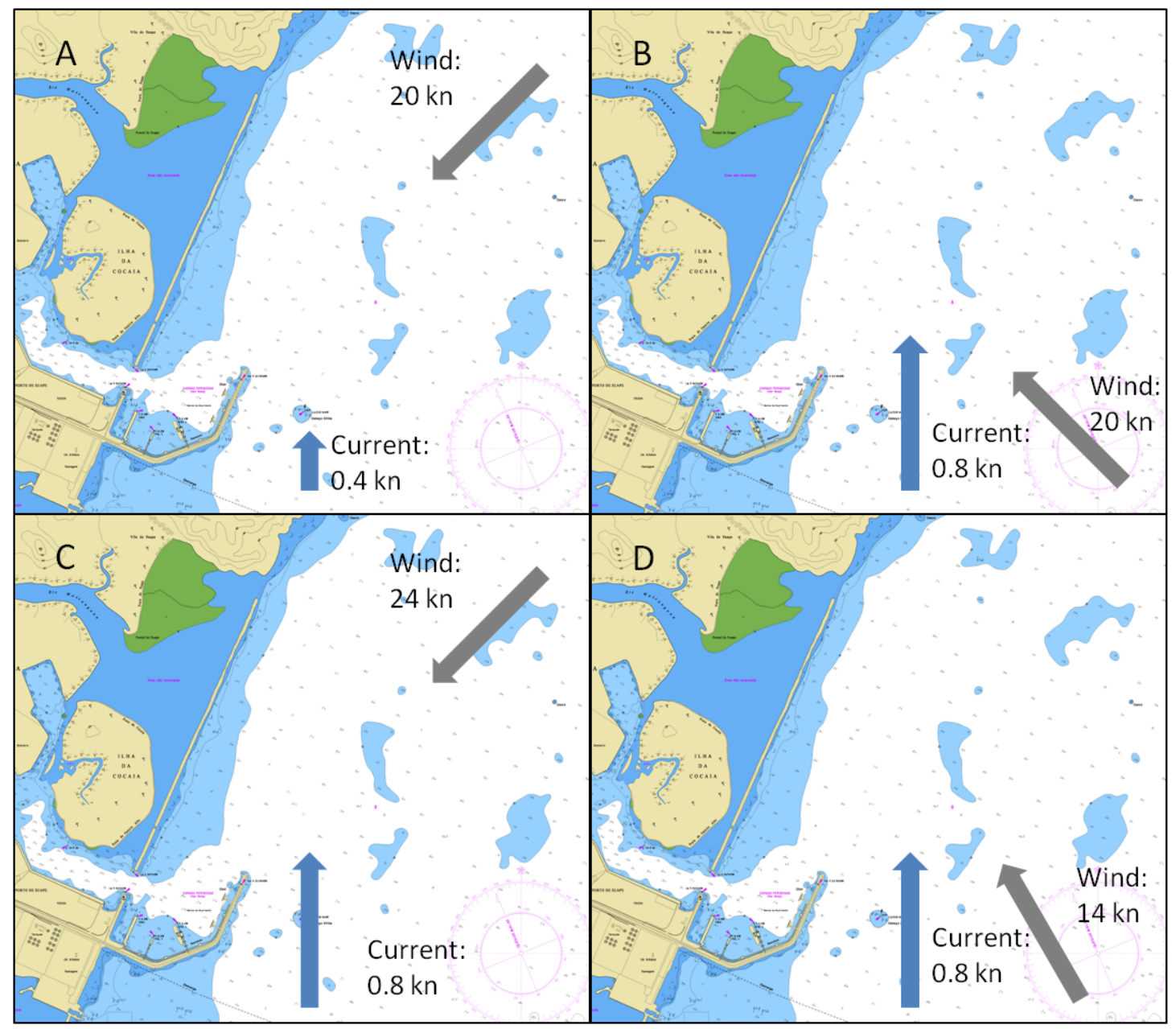

Figure 35: Visual representation of wind and current conditions evaluated in Suape Channel.A- N04W20, B-N08W20, C-N08W24, D-N08W20-330.

Figure 36 illustrates trajectories obtained by the policy using APE-X-DQN. As example of trajectory characteristics, further analysis were conducted using trajectory for 
Scenario N08W20.

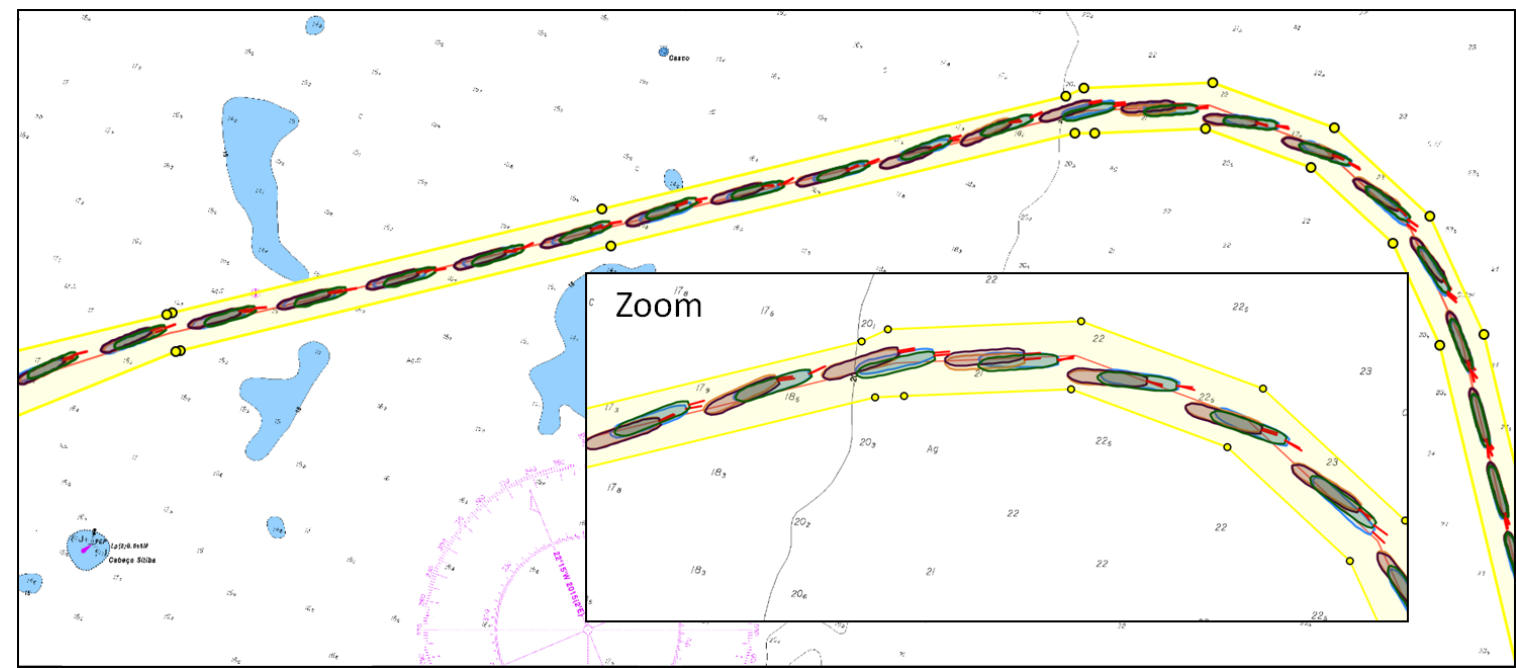

Figure 36: Trajectories for policy trained with APE-X-DQN. Blue ship: Scenario N04W20; Orange ship: Scenario N08W20-330; Purple ship:Scenario N08W20 , Green ship: Scenario N08W24.

Figures 37 and 38 depict vessel orientation and course over ground for N08W20 using policies obtained with APE-X-DQN and PPO respectively. The drift angle (difference between course over ground and heading) is smaller than $5^{\circ}$ for the straight portions for both cases. It is expected a higher drift angle during the curve due to the prolonged vessel orientation change. The policy for APE-X-DQN, however, presented higher overshooting in the final straight portion, specially for interval [1000, 1500].

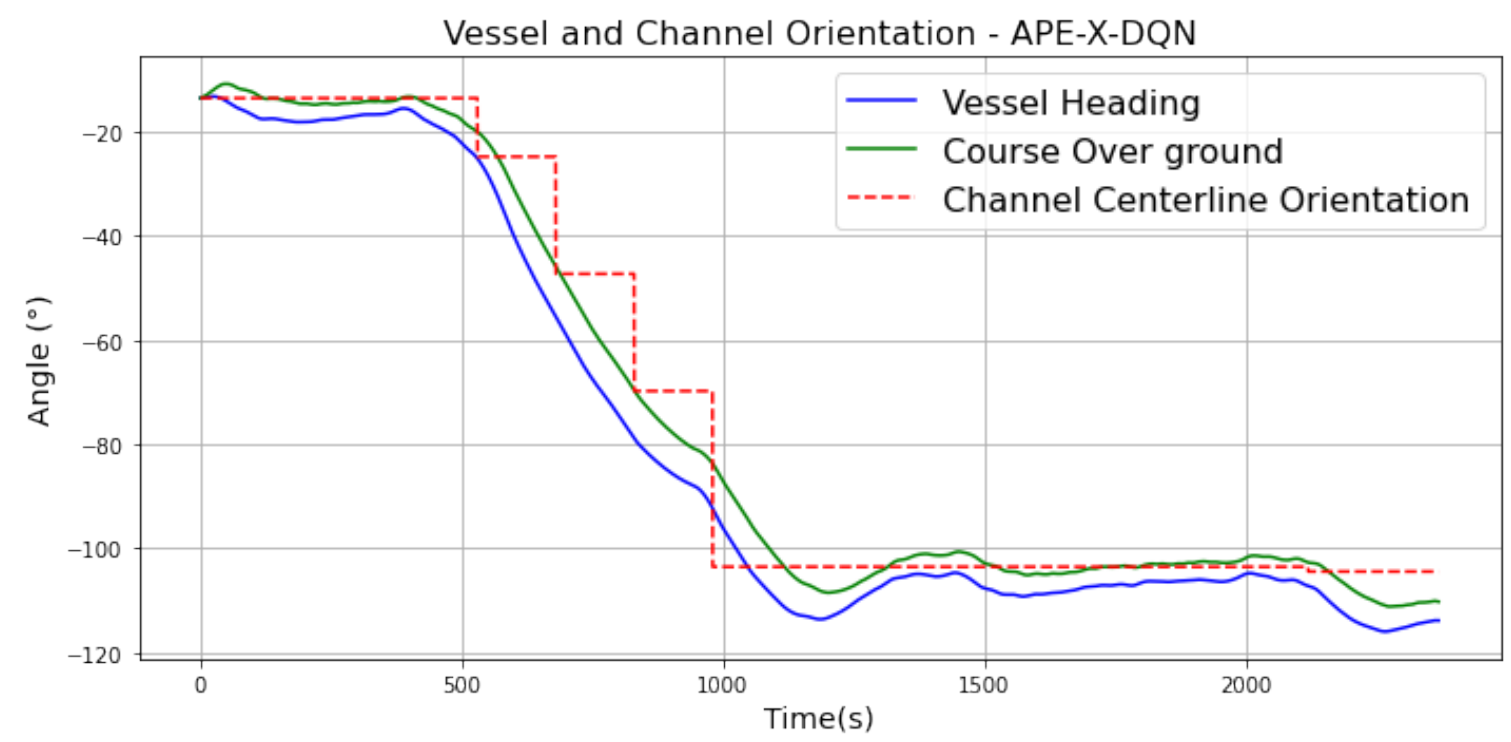

Figure 37: Vessel's heading and course over ground in Scenario N08W20 for APE-X-DQN 


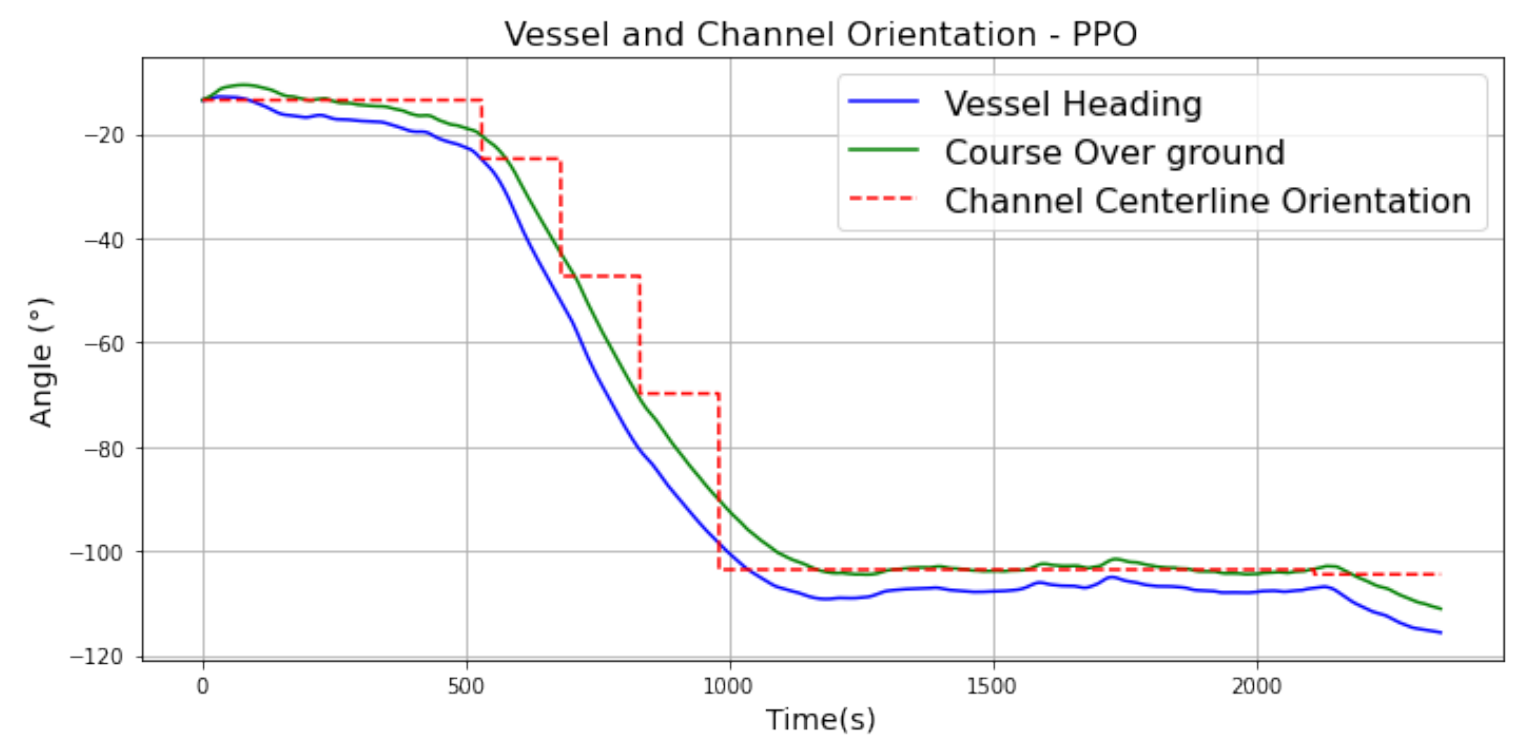

Figure 38: Vessel's heading and course over ground in Scenario N08W20 for PPO

Rudder commands are also displayed for both policies in Figures 39 and 40 in a 1000 s time window. As previously observed in Section 5.4, higher rudder angles are required for curve, with frequent angle changes in order to stabilize its orientation. The trajectory generated with APE-X-DQN, however, changed the rudder level commanded with lower frequency, which might explain the higher heading overshooting observed previously.

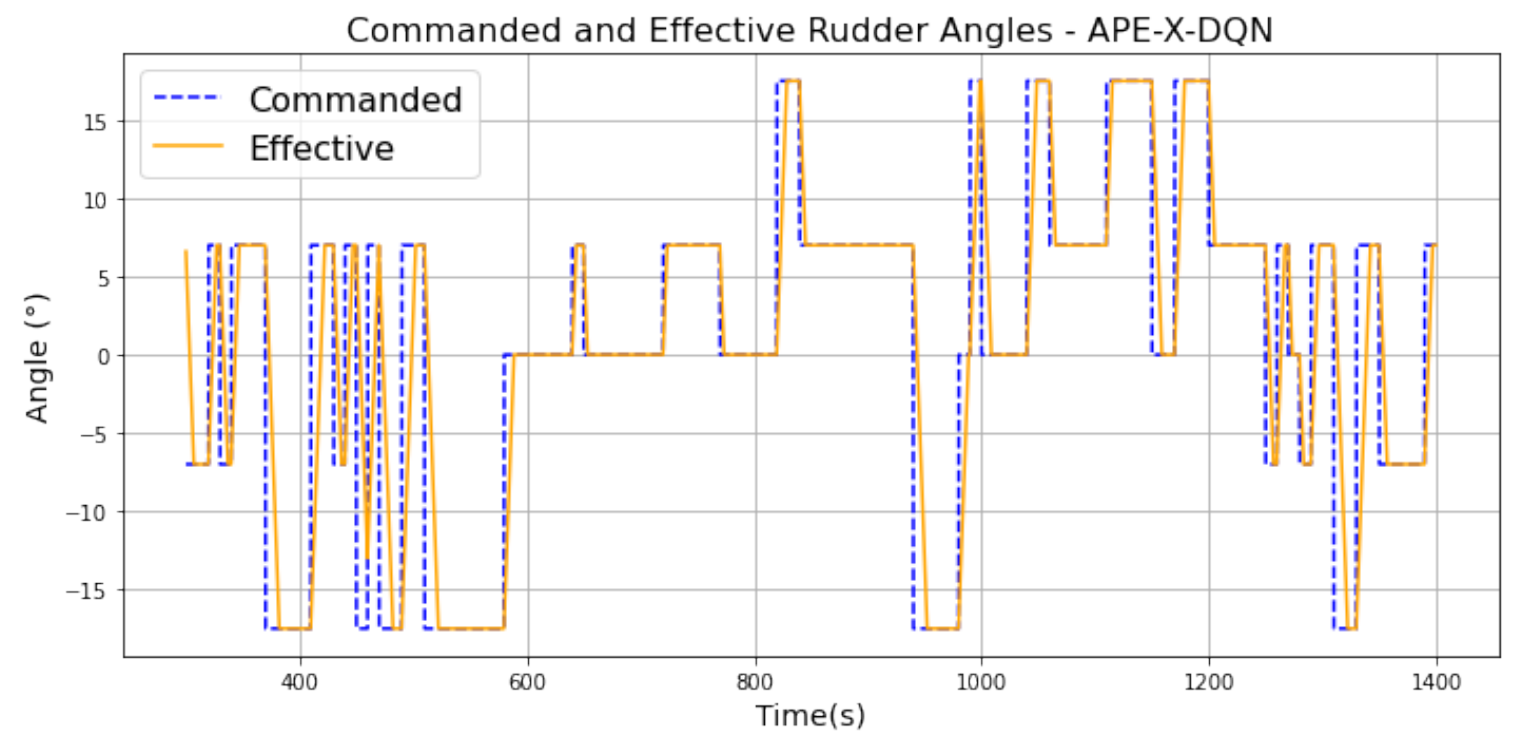

Figure 39: Rudder angles commanded by the agent and its effective value in Scenario N08W20 for APE-X-DQN 


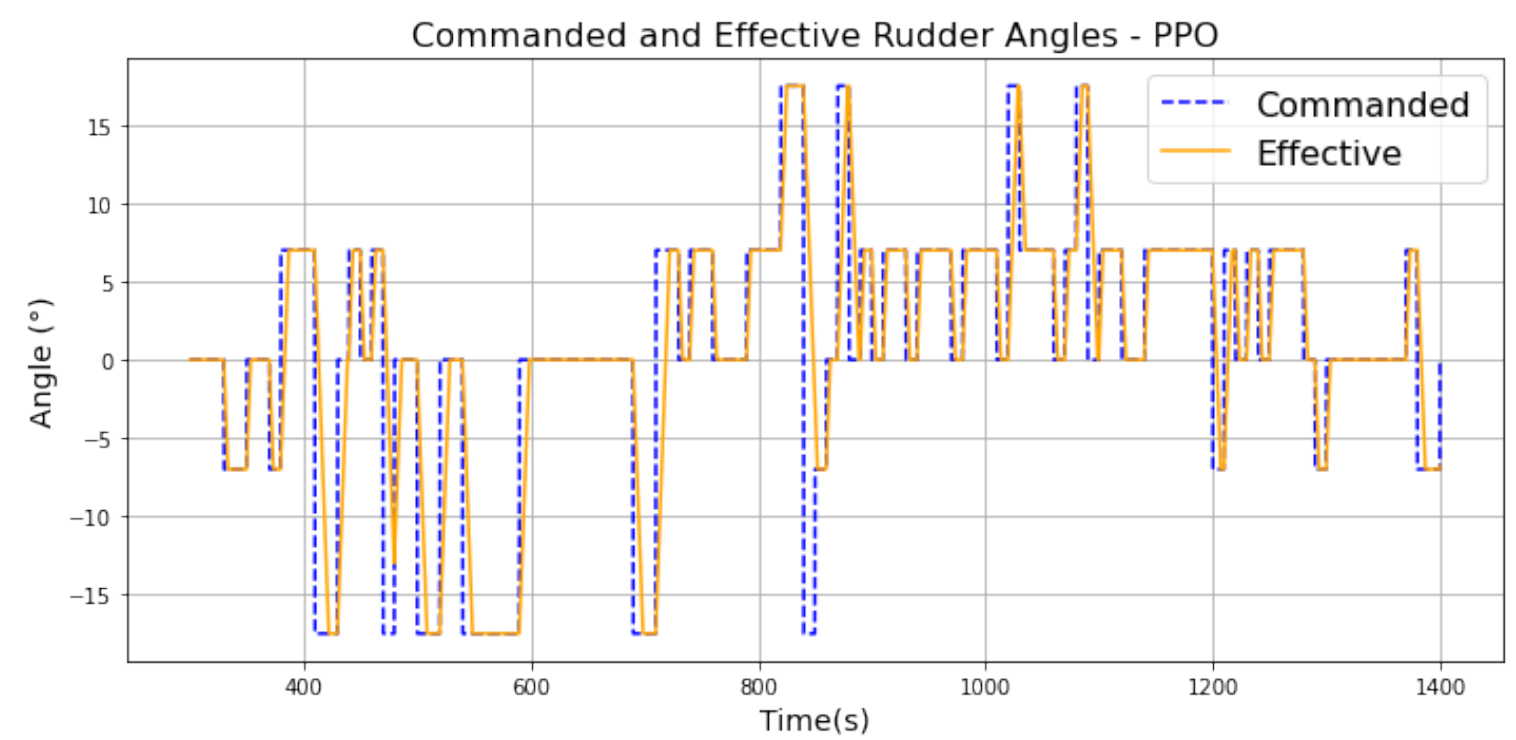

Figure 40: Rudder angles commanded by the agent and its effective value in Scenario N08W20 for PPO

Speed over ground for Scenario N08W20 are depicted for both policies in Figures 41 and 42. As expected, its value only reduces at the channel curved portion. Another decrease observed for APE-X-DQN in interval [1000, 1300] can be related to the lower rudder angle commutation during this period, causing a higher hydrodynamic resistance.

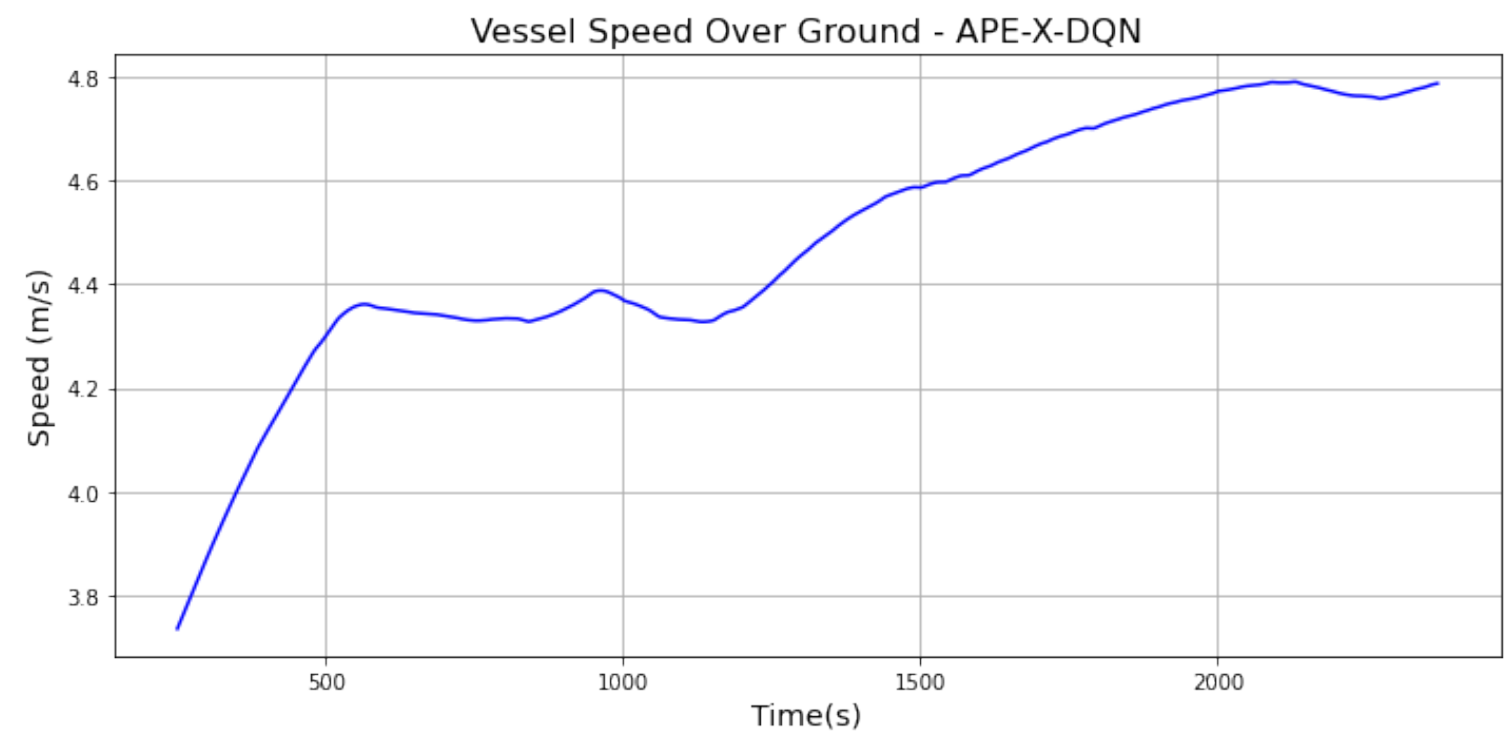

Figure 41: Speed over ground along the navigation in Scenario F05 using APE-X-DQN. 


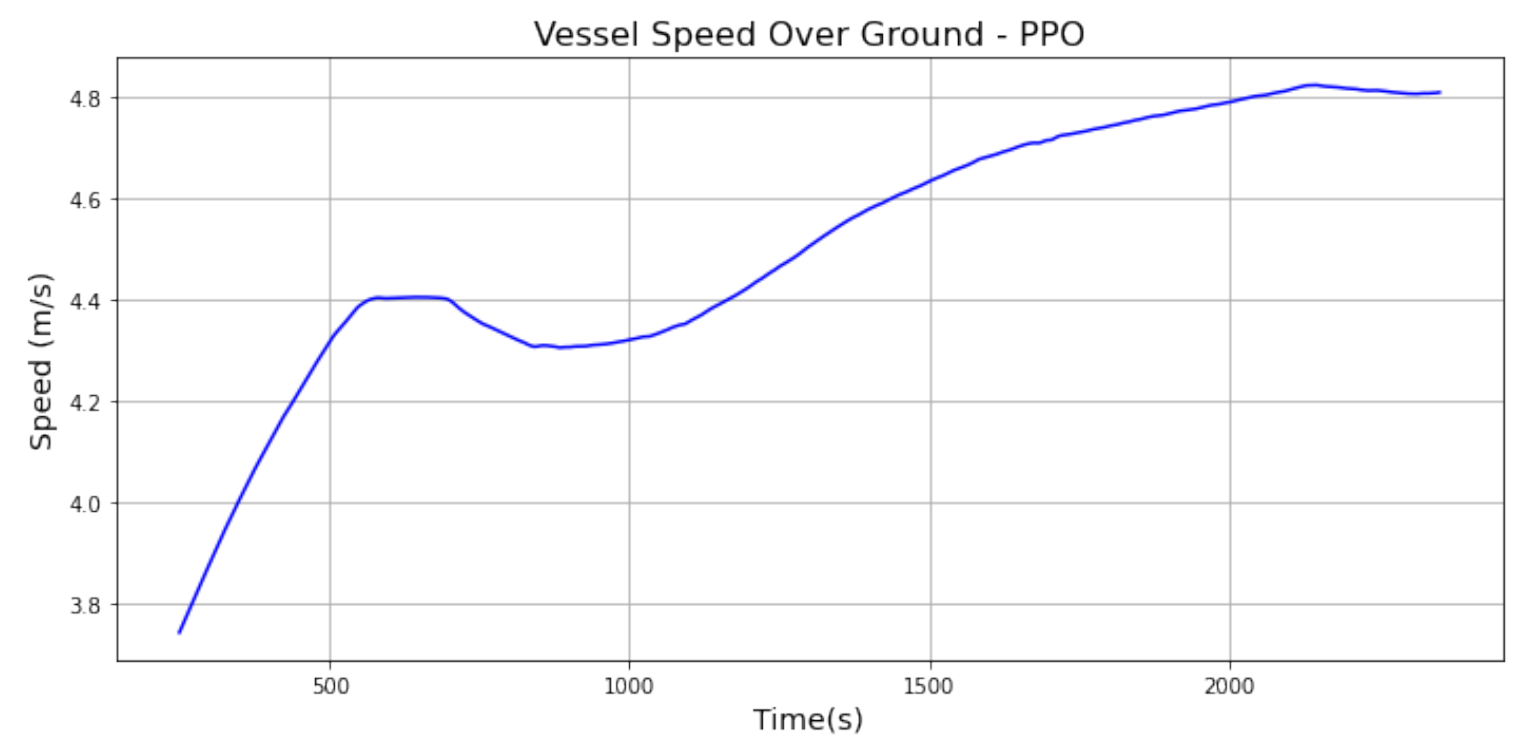

Figure 42: Speed over ground along the navigation in Scenario F05 using PPO.

Distances to margins are portrayed for all scenarios in Figures 43 and 44. The lowest distances occur during channel curved portion and, still, are mostly higher than $23 \mathrm{~m}$, except for Scenario N08W20-330 using APE-X-DQN.

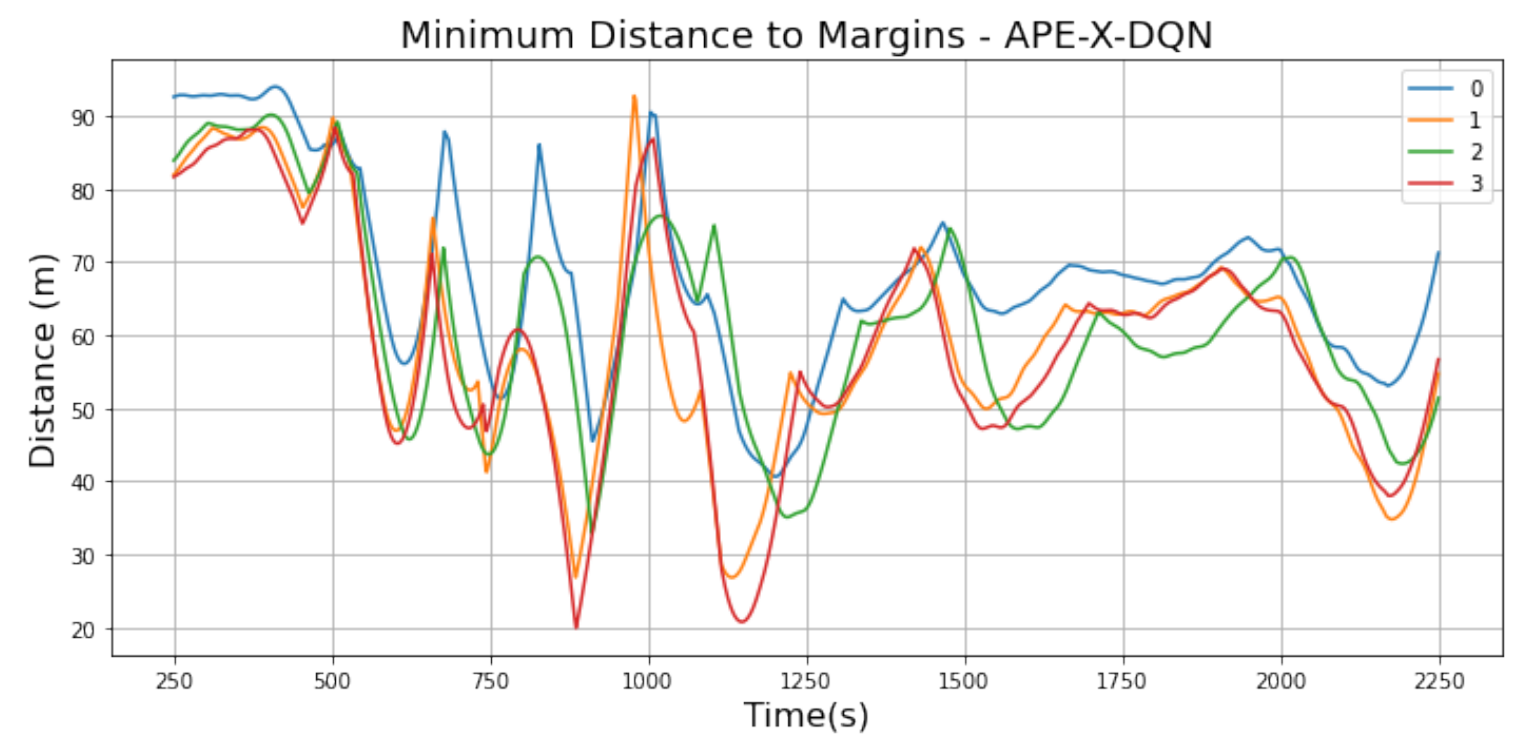

Figure 43: Distance to margins for scenarios using APE-X-DQN. 0-N04W20, 1-N08W20, 2-N08W24, 3-N08W20-330. 


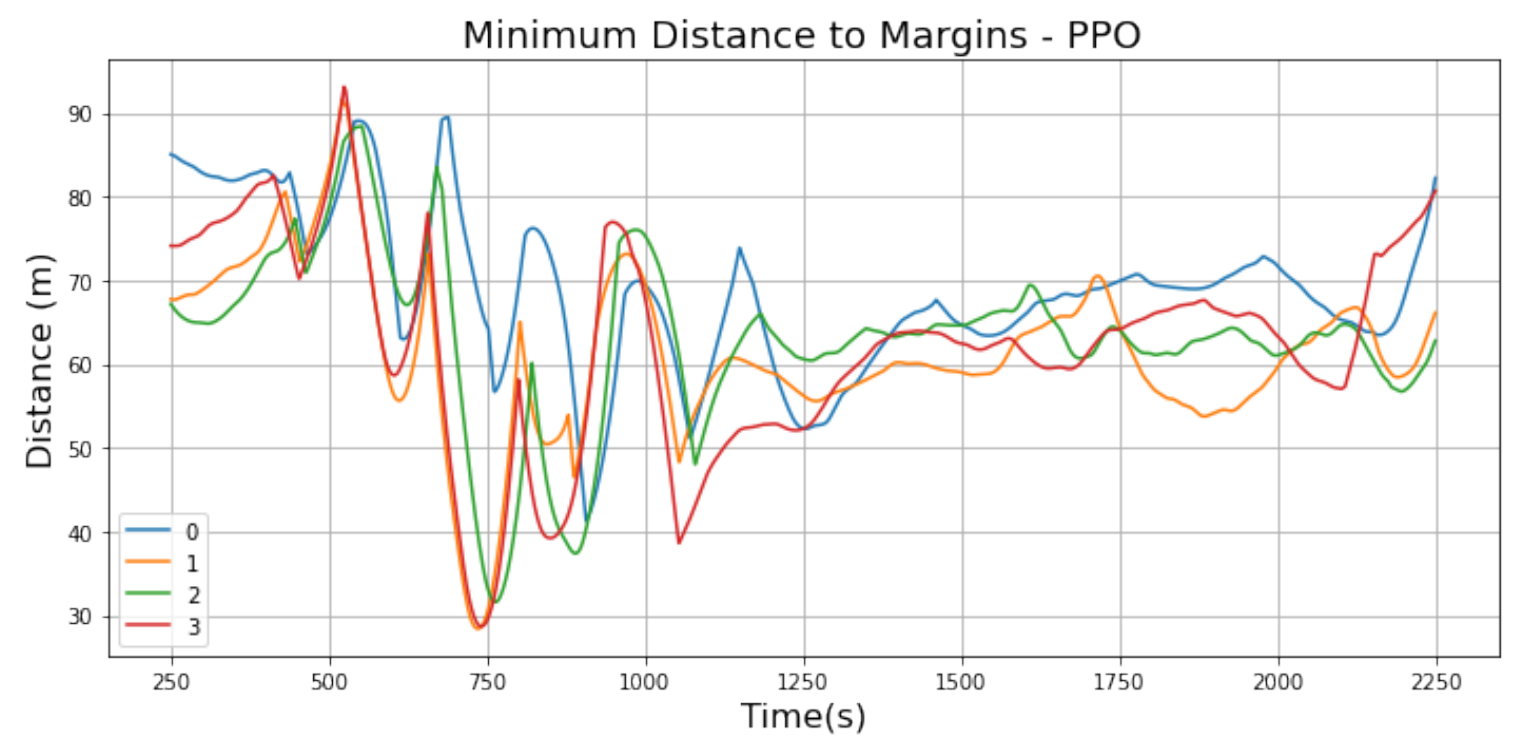

Figure 44: Distance to margins for scenarios using PPO. 0-N04W20, 1-N08W20, 2N08W24, 3-N08W20-330.

The policy was also successful in navigating in a channel with a prolonged curvature. In this case, APE-X-DQN policy generated trajectories with characteristics that are slightly worse than ones generated with PPO. Nonetheless, that analysis should not disqualify APE-X-DQN, since training occured under different conditions. The main attribute validated in this case is the capacity of extrapolation, which was accomplished in this case. Section 5.6 demonstrates that the policy can be improved for a specific channel.

\subsection{Training in Porto Sudeste Channel}

For a more thorough analysis, an experiment was conducted with trials using experiences only from Porto Sudeste channel. Experiment details and results were published in (Amendola et al., 2020). The environmental scenarios adopted during training for experience collection were E04, E08, F04 and F08.

Figure 45 shows the vessel course over ground and heading for a trajectory under scenario F08. The policy was trained with APE-X-DQN. In this case, even with a current intensity higher than F05, the heading angle in relation to channel orientation is clearly more satisfactory than observed in Figure 25. The vessel could anticipate channel orientation changes with a much lower overshooting, as for interval [1200, 1500] s. 


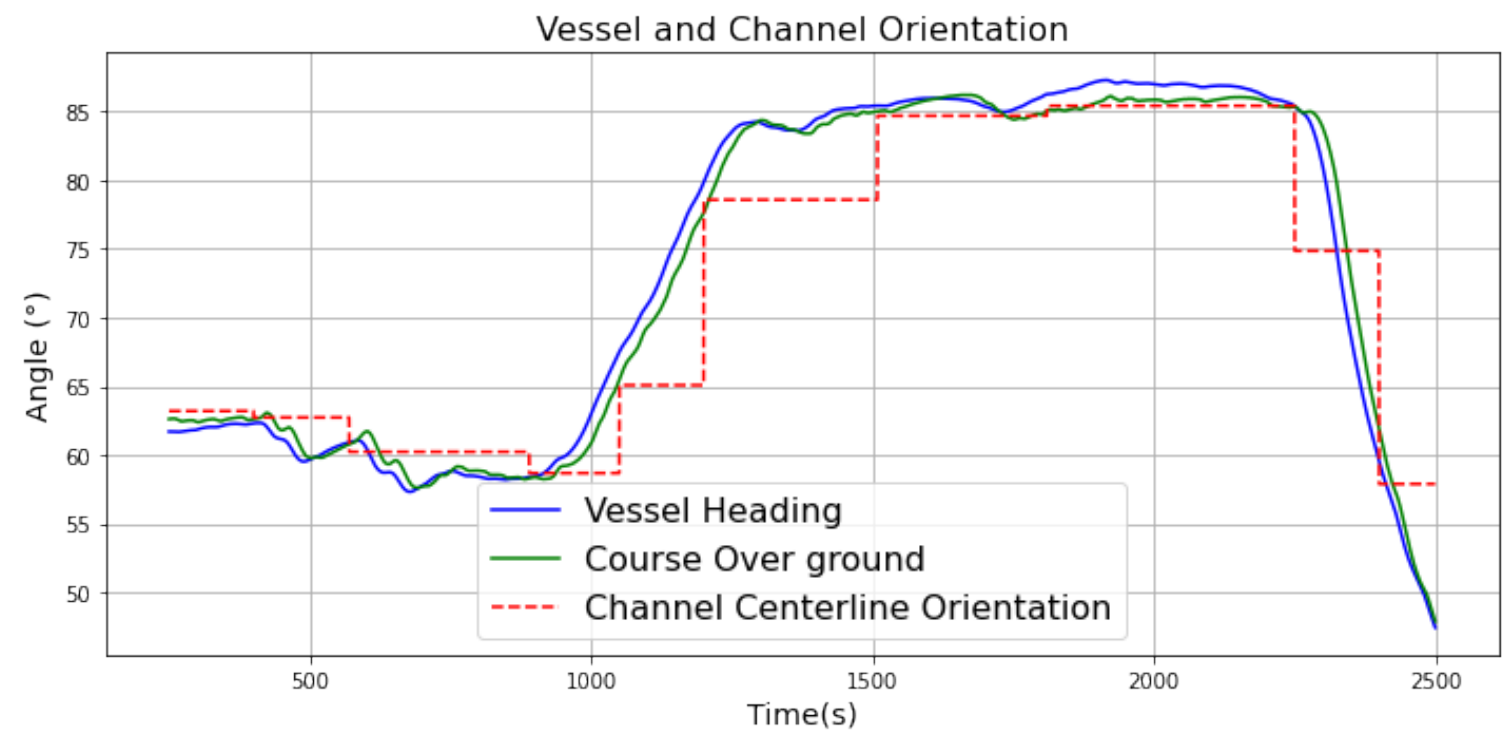

Figure 45: Vessel's heading and course over ground in Scenario F08 for APE-X-DQN training in Porto Sudeste

The distance to margins for the same trajectory in F08 is depicted in 46. When compared to distances in Figure 31, it is possible to see that the trajectory presented is safer. The minimum distance is higher than the minimum obtained with generic policy for scenario F08 and other scenarios with even lower current.

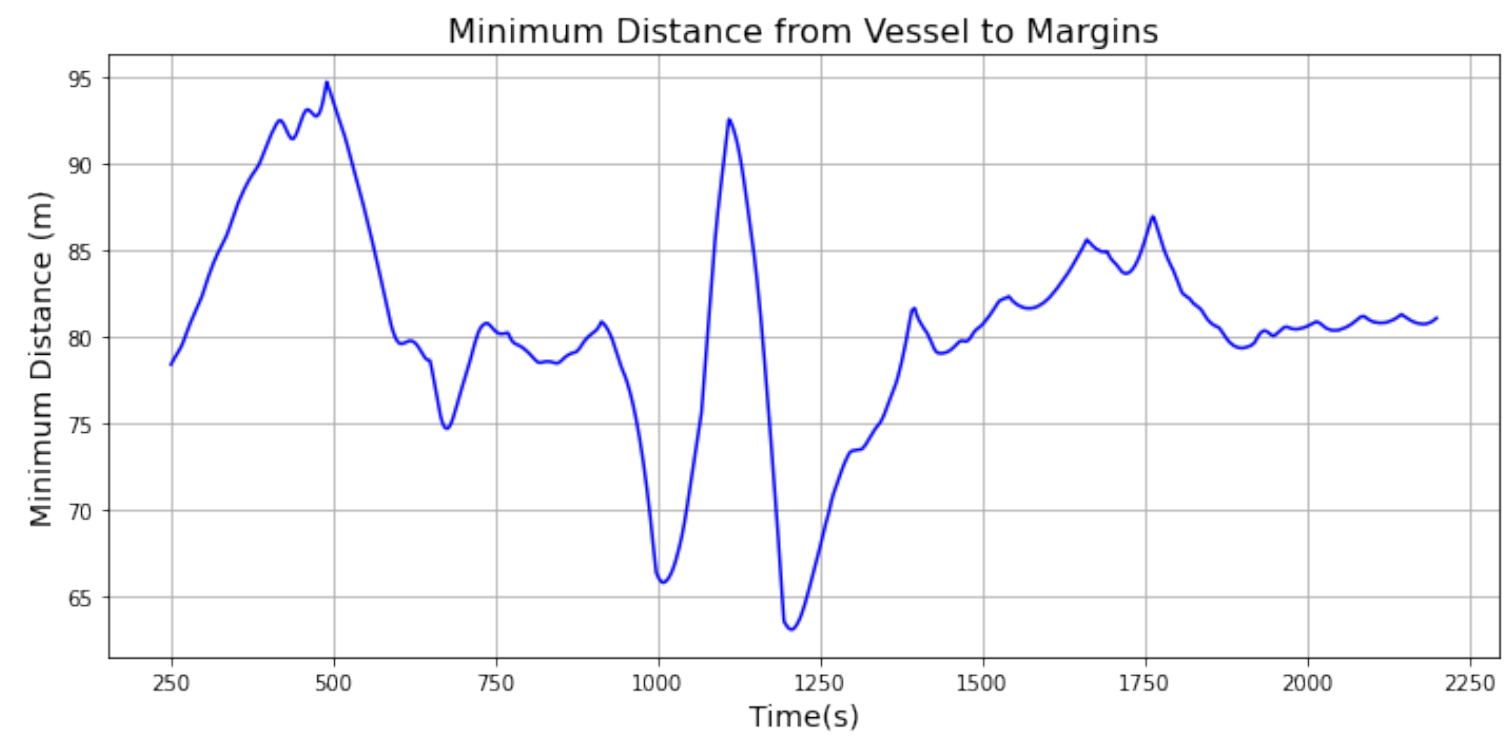

Figure 46: Distance to margins in Scenario F08 for APE-X-DQN training in Porto Sudeste

Training for a specific channel under a restricted number of scenarios has shown that it is possible to obtain a policy with higher performance than the generic one. That occurs because the curvature and environment conditions used during trial exactly match the experiences of the evaluated scenario. 
There is a trade-off between generalization capacity and trajectory optimization. In practice, a middle ground solution would be the definition of a generic policy, as in Section 5.3 , and further training of this policy under specific scenario on demand. This technique is also called Transfer Learning. 


\section{CONCLUSION AND NEXT STEPS}

This work developed a reinforcement learning based solution for trajectory generation in fast-time simulations of navigation in restricted port channels. The solution can handle narrow curves and environmental forces that might affect the vessel differently as channel orientation changes. requiring hand-crafted way-points and the tuning of control algorithms, presenting a human-labor efficient solution. It is quite a compact representation that enabled human-style navigation through discrete rudder levels and discrete interval between actions.

The adopted algorithms resorted to substantial parallel computing resources. After training for a set of representative navigation scenarios, the policy learned to navigate in different channels and generalize its ability to a variety of environmental conditions. It was also demonstrated that a generic policy can be improved and optimized when trained for specific scenarios.

Both reinforcement learning algorithms revealed a trade-off: PPO was more stable but it does not provide estimated values for state-action pairs in a greedy policy. On the other hand, APE-X-DQN is value based can achieve a desirable policy faster, but training is more oscillatory. Still, performance relies on hyperparameters, so future efforts can involve hyper parameter optimization.

The solution can be further extended to the subsequent phases in simulated navigation, with formulations that include propulsion command for slow-down process and towing process. The dimensionality would increase and so the complexity of the problem.

In a long-term horizon, reinforcement learning paradigm can turn fast-time simulations more significant in project analysis context. Since the goal is to obtain a policy as close as human behavior in real piloting, other techniques could cope for finding reward functions which could maximize this similarity, even for specific pilots, as in the so-called inverse reinforcement learning (FU; LUO; LEVINE, 2017) (ARORA; DOSHI, 2018). Also, other innovative approaches which combine partial human feedback with 
reinforcement learning (KNOX; STONE, 2012) (GRIFFITH et al., 2013) (CRUZ et al., 2018) can be explored in the domain of this work. Assuming the pilot well represented by a policy, criticality of navigation might consider emotion models from values estimated in reinforcement learning, as in robotics (MOERLAND; BROEKENS; JONKER, 2018).

Although the present work focuses on fast-time simulations, where obstacles are static, future work must also consider two-way channels with passing vessels, with further analysis on safety so that the model can be transferred to autonomous marine vehicles. 
Appendices 


\section{A NEURAL NETWORKS}

An Artificial Neural Network (ANN) (HAYKIN, 1999), as depicted in Figure 47, is an efficient computing system whose central theme is borrowed from the analogy of biological neural networks. ANNs are also named as "artificial neural systems," or "parallel distributed processing systems," or "connectionist systems." They constitute a collection of units that are interconnected in some pattern to allow communication between the units. These units, also referred to as nodes or neurons, are simple processors which operate in parallel.

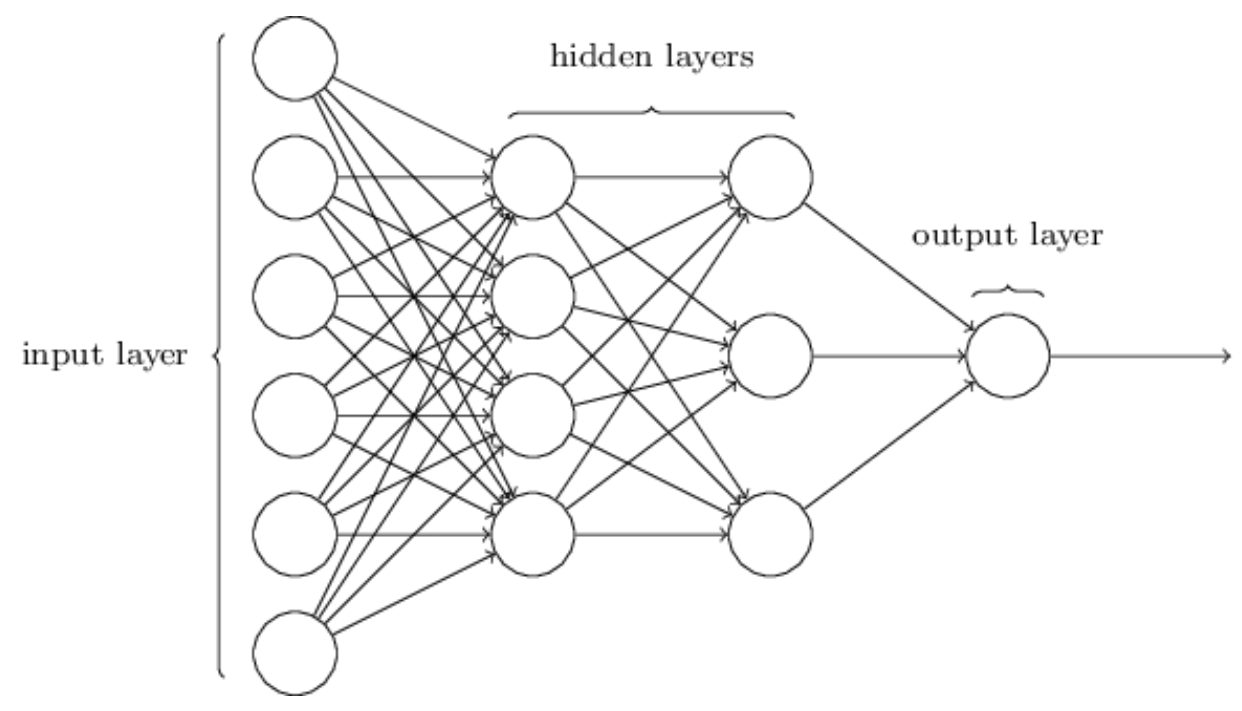

Figure 47: Neural network

Every neuron is connected with another neuron through a connection link. Each connection link is associated with a weight that has information about the input signal. This is the most useful information for neurons to solve a particular problem because the weight usually excites or inhibits the signal that is being communicated. As displayed in Figure 48, each neuron has an internal state, which is called an activation signal. Output signals, which are produced after combining the input signals and activation rule, may be sent to other units.

Although very often applied to classification tasks, neural networks can be considered 


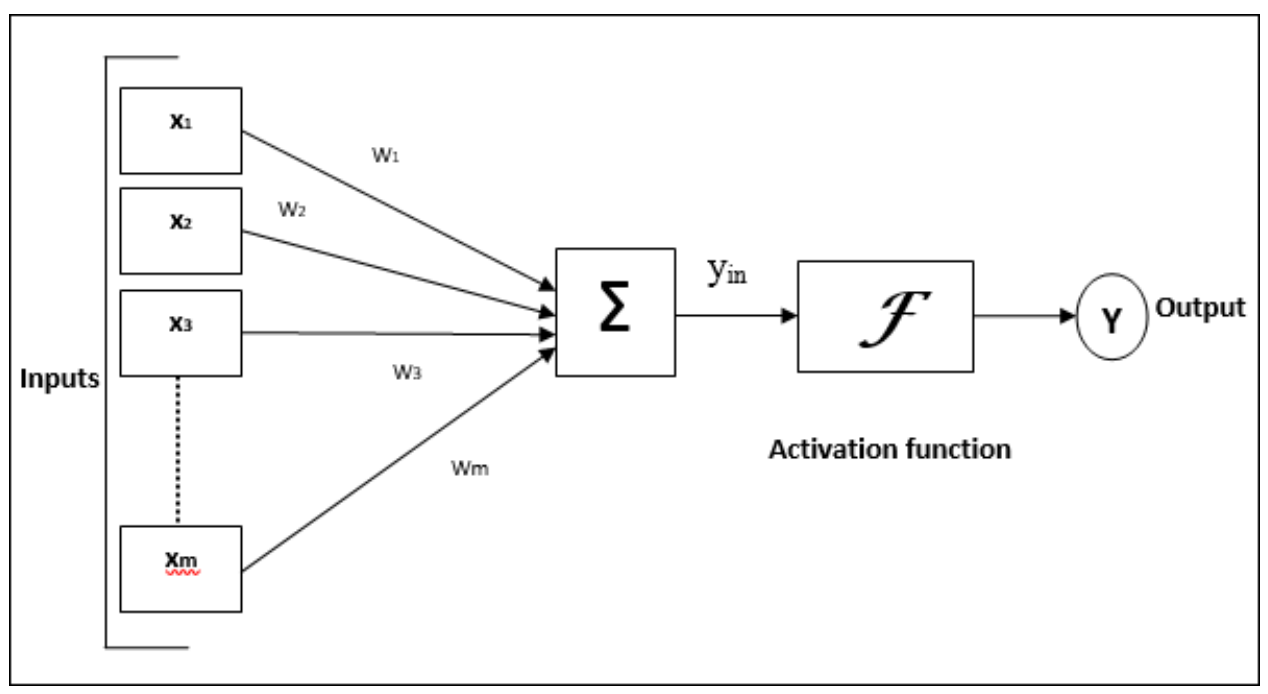

Figure 48: Neuron basic scheme

non-linear function approximators. In the mathematical theory of ANNs, the universal approximation theorem states that a feed-forward network with a single hidden layer containing a finite number of neurons can approximate any continuous function under mild assumptions on the activation function (CYBENKO, 1989). The theorem, thus, states that simple neural networks can represent a wide variety of interesting functions when given appropriate parameters. However, it does not address the algorithmic learnability of those parameters.

One of the first versions of the theorem was proved by George Cybenko in 1989 (CYBENKO, 1989) for sigmoid activation functions, given by:

$$
h(x)=\frac{1}{1+e^{-x}}
$$

The curve for the function is given in Figure 49 .

It was shown in 1991 (HORNIK, 1991) that it is not the specific choice of the activation function, but rather the multilayer feedforward architecture itself which gives neural networks the potential of being universal approximators.

The learning process in ANNs is performed by adjusting the weights of connections. It occurs in two phases: forward propagation and backpropagation as illustrated in Figure 50 .

In forward propagation the network is exposed to the training data and these cross the entire neural network for their predictions (labels) to be calculated. That is, passing the input data through the network in such a way that all the neurons apply their 


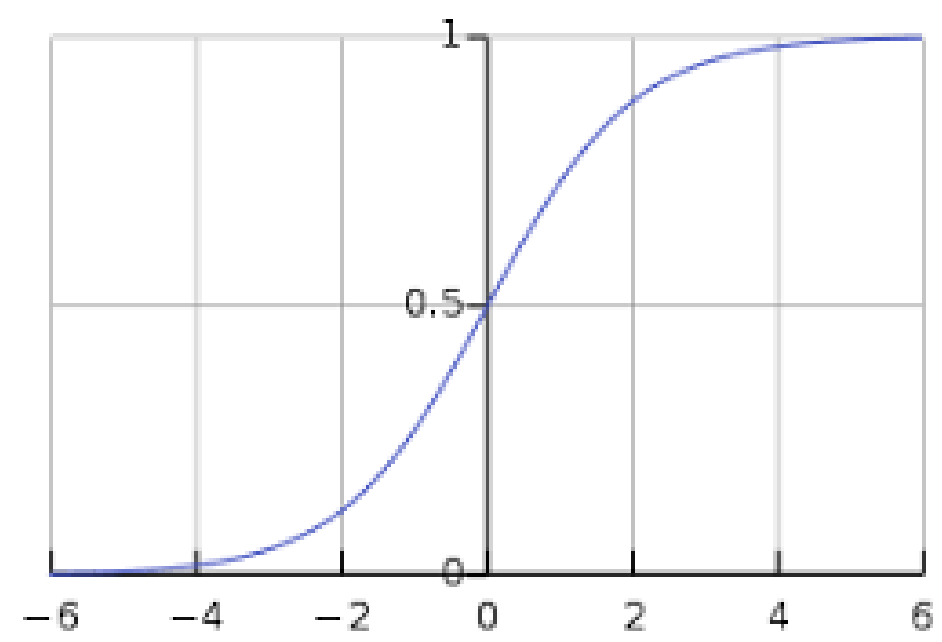

Figure 49: Sigmoidal function

transformation to the information they receive from the neurons of the previous layer and sending it to the neurons of the next layer.

Next, the loss function (or error) measures how good the prediction result was in relation to the correct result which was supplied (also known as label in classification tasks or target in approximation tasks). The weights of the interconnections of the neurons will gradually be adjusted until good predictions are obtained.

Once the loss has been calculated, this information is propagated backwards. Hence, its name: backpropagation. Starting from the output layer, that loss information propagates to all the neurons in the hidden layer that contribute directly to the output. However, the neurons of the hidden layer only receive a fraction of the total signal of the loss, based on the relative contribution that each neuron has given to the original output. This process is repeated, layer by layer, until all the neurons in the network have received a loss signal that describes their relative contribution to the total loss.

After that information was spread back, the weights of connections must be adjusted with the goal of reducing loss function. This is performed by a technique called gradient descent. This technique changes the weights in small increments with the help of the calculation of the derivative (or gradient) of the loss function, which allows to see in which direction "to descend" towards the global minimum; this is usually done in batches of data in the successive iterations (epochs) of all the data set that are passed to the network in each iteration. The update for each weight is given by:

$$
W_{i}^{\text {new }}=W_{i}^{\text {old }}-\eta \frac{\partial L(y, \hat{y})}{\partial W_{i}^{\text {old }}}
$$




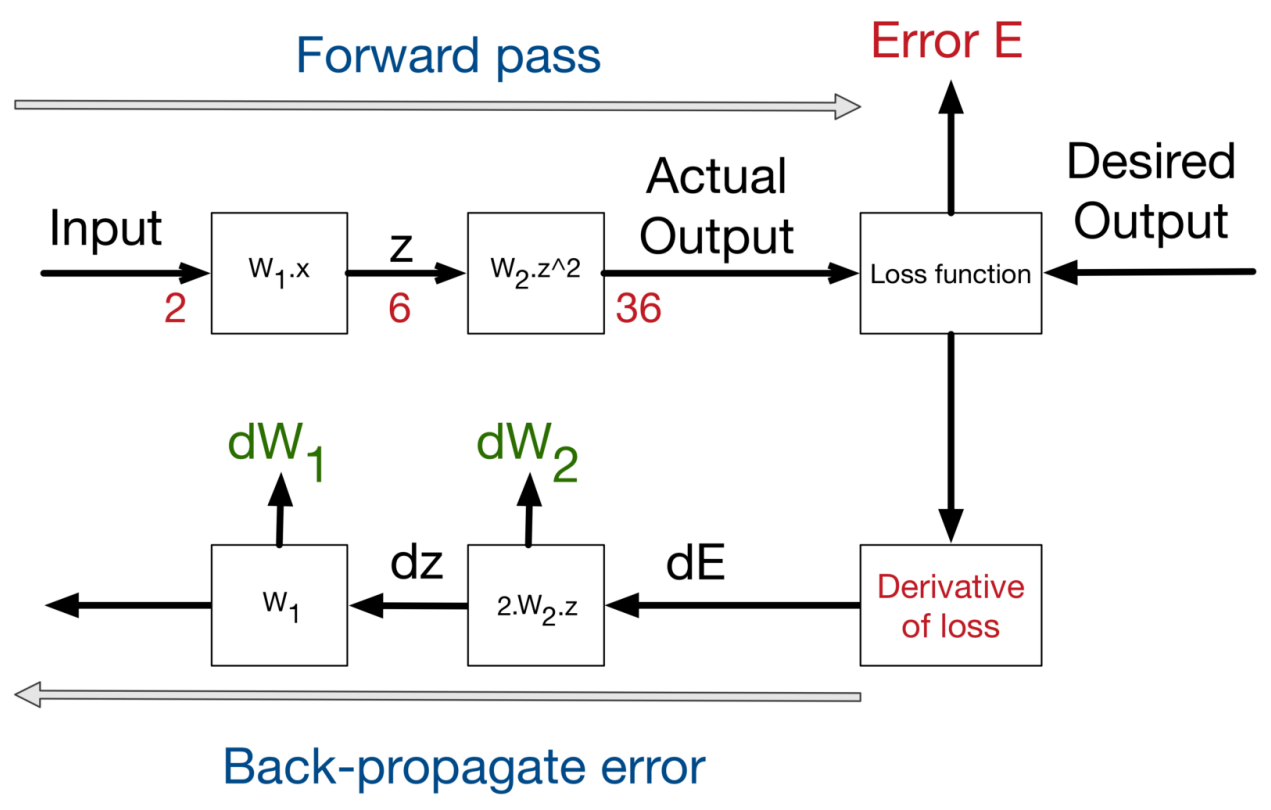

Figure 50: Forward propagation and Backpropagation

where $W_{i}^{\text {new }}$ is the new value of the parameter, $W_{i}^{\text {old }}$ is the old value, $\eta$ is the learning rate and $L(y, \hat{y})$ is the loss function. 


\section{REFERENCES}

Amendola, J.; Miura, L. S.; Costa, A. H. R.; Cozman, F. G.; Tannuri, E. A. Navigation in restricted channels under environmental conditions: Fast-time simulation by asynchronous deep reinforcement learning. IEEE Access, p. 1-1, 2020.

AMENDOLA, J.; TANNURI, E. A.; COZMAN, F. G.; COSTA, A. H. R. Batch reinforcement learning of feasible trajectories in a ship maneuvering simulator. Anais do Encontro Nacional de Inteligência Artificial e Computacional (ENIAC), p. 263-274, 2018. Disponível em:〈http://portaldeconteudo.sbc.org.br/index.php/eniac/article/ view $/ 4422\rangle$.

AMENDOLA, J.; TANnURI, E. A.; COZMAN, F. G.; COSTA, A. H. R. Port channel navigation subjected to environmental conditions using reinforcement learning. In: AMERICAN SOCIETY OF MECHANICAL ENGINEERS. International Conference on Offshore Mechanics and Arctic Engineering. [S.l.], 2019. v. 58844, p. V07AT06A042.

ANTOS, A.; SZEPESVÁRI, C.; MUNOS, R. Fitted q-iteration in continuous actionspace mdps. In: Advances in neural information processing systems. [S.l.: s.n.], 2008. p. $9-16$.

ARORA, S.; DOSHI, P. A survey of inverse reinforcement learning: Challenges, methods and progress. arXiv preprint arXiv:1806.06877, 2018.

BELLMANN, R. Dynamic programming princeton university press. Princeton, NJ, 1957.

BENEDICT, K.; FISCHER, S.; GLUCH, M.; KIRCHHOFF, M.; SCHAUB, M.; BALDAUF, M.; MÜLLER, B. Innovative fast time simulation tools for briefing/debriefing in advanced ship handling simulator training and ship operation. Transactions on maritime science, Pomorski fakultet u Splitu, v. 6, n. 01, p. 24-38, 2017.

BHATT, A. N.; ARGUS, M.; AMIRANASHVILI, A.; BROX, T. Crossnorm:

Normalization for off-policy td reinforcement learning. ArXiv, abs/1902.05605, 2019.

BISHOP, C. M. Pattern recognition and machine learning. Springer, 2013.

CHEN, C.; CHEN, X.-Q.; MA, F.; ZENG, X.-J.; WANG, J. A knowledge-free path planning approach for smart ships based on reinforcement learning. Ocean Engineering, Elsevier, v. 189, p. 106299, 2019.

CHENG, Y.; ZHANG, W. Concise deep reinforcement learning obstacle avoidance for underactuated unmanned marine vessels. Neurocomputing, Elsevier, v. 272, p. 63-73, 2018.

CRUZ, F.; MAGG, S.; NAGAI, Y.; WERMTER, S. Improving interactive reinforcement learning: What makes a good teacher? Connection Science, Taylor \& Francis, v. 30, n. 3, p. 306-325, 2018. 
CYBENKO, G. Approximation by superpositions of a sigmoidal function. Mathematics of Control, Signals and Systems, v. 2, n. 4, p. 303-314, Dec 1989. ISSN 1435-568X. Disponível em: 〈https://doi.org/10.1007/BF02551274〉.

DULAC-ARNOLD, G.; MANKOWITZ, D.; HESTER, T. Challenges of real-world reinforcement learning. arXiv preprint arXiv:1904.12901, 2019.

FOSSEN, T. I. Handbook of Marine Craft Hydrodynamics and Motion Control. Chichester, UK: John Wiley \& Sons, Ltd, 2011. ISBN 9781119994138. Disponível em: 〈http://doi.wiley.com/10.1002/9781119994138〉.

FU, J.; LUO, K.; LEVINE, S. Learning robust rewards with adversarial inverse reinforcement learning. arXiv preprint arXiv:1710.11248, 2017.

GRIFFITH, S.; SUBRAMANIAN, K.; SCHOLZ, J.; ISBELL, C. L.; THOMAZ, A. L. Policy shaping: Integrating human feedback with reinforcement learning. In: Advances in neural information processing systems. [S.1.: s.n.], 2013. p. 2625-2633.

GUO, S.; ZHANG, X.; ZHENG, Y.; DU, Y. An autonomous path planning model for unmanned ships based on deep reinforcement learning. Sensors, Multidisciplinary Digital Publishing Institute, v. 20, n. 2, p. 426, 2020.

HAFNER, R.; RIEDMILLER, M. Reinforcement learning in feedback control. Machine learning, Springer, v. 84, n. 1-2, p. 137-169, 2011.

HAFNER, R.; RIEDMILLER, M. Reinforcement learning in feedback control. Machine Learning, v. 84, n. 1, p. 137-169, Jul 2011. ISSN 1573-0565. Disponível em: $\langle$ https://doi.org/10.1007/s10994-011-5235-x〉.

HAYKIN, S. Neural Networks: A Comprehensive Foundation. Prentice Hall, 1999. (International edition). ISBN 9780139083853. Disponível em: 〈https: //books.google.com.br/books?id=M5abQgAACAAJ $\rangle$.

HENDERSON, P.; ISLAM, R.; BACHMAN, P.; PINEAU, J.; PRECUP, D.; MEGER, D. Deep reinforcement learning that matters. In: Thirty-Second AAAI Conference on Artificial Intelligence. [S.l.: s.n.], 2018.

HORGAN, D.; QUAN, J.; BUDDEN, D.; BARTH-MARON, G.; HESSEL, M.; HASSELT, H. V.; SILVER, D. Distributed prioritized experience replay. arXiv preprint arXiv:1803.00933, 2018.

HORNIK, K. Approximation capabilities of multilayer feedforward networks. Neural Networks, v. 4, n. 2, p. 251 - 257, 1991. ISSN 0893-6080. Disponível em: 〈http://www.sciencedirect.com/science/article/pii/089360809190009T〉.

KAMIO, T.; MITSUBORI, K.; TANAKA, T.; FUJISAKA, H.; HAEIWA, K. Effects of prior knowledge on multi-agent reinforcement leaning system to find courses of ships. Australian Journal of Intelligent Information Processing Systems, v. 12, n. 2, 2010.

KINGMA, D. P.; BA, J. Adam: A method for stochastic optimization. In: BENGIO, Y.; LECUN, Y. (Ed.). 3rd International Conference on Learning Representations, ICLR 2015, San Diego, CA, USA, May 7-9, 2015, Conference Track Proceedings. [s.n.], 2015. Disponível em: 〈http://arxiv.org/abs/1412.6980〉. 
KNOX, W. B.; STONE, P. Reinforcement learning from simultaneous human and mdp reward. In: INTERNATIONAL FOUNDATION FOR AUTONOMOUS AGENTS AND MULTIAGENT SYSTEMS. Proceedings of the 11th International Conference on Autonomous Agents and Multiagent Systems-Volume 1. [S.l.], 2012. p. 475-482.

KOBER, J.; BAGNELL, J. A.; PETERS, J. Reinforcement learning in robotics: A survey. The International Journal of Robotics Research, SAGE Publications Sage UK: London, England, v. 32, n. 11, p. 1238-1274, 2013.

KOOIJ, C.; COLLING, A.; BENSON, C. When will autonomous ships arrive? a technology forecasting perspective. INEC Conference 2018, 2018.

LACKI, M. Reinforcement Learning in Ship Handling. TransNav, the International Journal on Marine Navigation and Safety of Sea Transportation, Gdynia Maritime University, Faculty of Navigation, v. 2, n. 2, p. 157-160, 2008. ISSN 2083-6473. Disponível em:〈./Article\_Reinforcement\_Learning \_in\_Ship \_Lacki,6,86.html〉.

LANGE, S.; GABEL, T.; RIEDMILLER, M. Batch reinforcement learning. In: Reinforcement learning. [S.l.]: Springer, 2012. p. 45-73.

LAURINEN, M. Remote and Autonomous Ships: The next steps. 2016. 88 p. Disponível em: 〈http://www.rolls-royce.com/\{ \}/media/Files/R/Rolls-Royce/documents/ customers/marine/ship-intel/aawa-whitepaper-210616.p $\rangle$.

LIANG, E.; LIAW, R.; MORITZ, P.; NISHIHARA, R.; FOX, R.; GOLDBERG, K.; GONZALEZ, J. E.; JORDAN, M. I.; STOICA, I. Rllib: Abstractions for distributed reinforcement learning. arXiv preprint arXiv:1712.09381, 2017.

LIXING, Z.; LEI, Q.; JIANLIANG, C.; WEIDONG, Z. Neural-network-based reinforcement learning control for path following of underactuated ships. 2016 35th Chinese Control Conference (CCC), p. 5786-5791, 2016.

LLOYD J.; RODRIGUES, I. Simulation, an effective tool in the risk management of vessels under pilotage. In: International Conference on Marine Simulation and Ship Manoeuvrability. [S.l.: s.n.], 2012. p. 749-757.

MAAS, A. L.; HANNUN, A. Y.; NG, A. Y. Rectifier Nonlinearities Improve Neural Network Acoustic Models. In: International conference on machine learning. [s.n.], 2013. Disponível em: 〈https://pdfs.semanticscholar.org/367f/ 2c63a6f6a10b3b64b8729d601e69337ee3cc.pdf $\rangle$.

MARTINSEN, A. B.; LEKKAS, A. M. Curved path following with deep reinforcement learning: Results from three vessel models. In: OCEANS 2018 MTS/IEEE Charleston. [S.l.: s.n.], 2018. p. 1-8. ISSN 0197-7385.

MARTINSEN, A. B.; LEKKAS, A. M. Straight-path following for underactuated marine vessels using deep reinforcement learning. IFAC-PapersOnLine, Elsevier BV, v. 51, n. 29, p. 329-334, 2018. Disponível em:〈https://doi.org/10.1016/j.ifacol.2018.09.502〉.

MCBRIDE, M.; BRIGGS, M.; GROENVELD, R.; BOLL, M.; CAO, L.; COCKRILL, D.; DEBAILlON, P.; DIETZE, W.; HARTIKAINEN, J.; IRIBARREN, J.; MOES, H.; O'BRIEN, T.; OHTSU, K.; PATEL, S.; FERNANDEZ, C.; SCHERRER, P.; SIRKIä, 
E.; TSUGAnE, M.; MOLEN, W. Van der; VANTORRE, M. PIANC Report No. 121 - 2014, Harbour Approach Channels - Design Guidelines. [S.l.: s.n.], 2014. ISBN 978-2-87223-210-9.

MEYER, E.; ROBINSON, H.; RASHEED, A.; SAN, O. Taming an autonomous surface vehicle for path following and collision avoidance using deep reinforcement learning. IEEE Access, IEEE, 2020.

MITSUBORI, K.; KAMIO, T.; TANAKA, T. Finding the shortest course of a ship based on reinforcement learning algorithm. The Journal of Japan Institute of Navigation, Japan Institute of Navigation, v. 110, p. 9-18, 2004.

Mnih, V.; Kavukcuoglu, K.; Silver, D.; Rusu, A. A.; Veness, J.; Bellemare, M. G.; Graves, A.; Riedmiller, M.; Fidjeland, A. K.; Ostrovski, G.; Petersen, S.; Beattie, C.; Sadik, A.; Antonoglou, I.; King, H.; Kumaran, D.; Wierstra, D.; Legg, S.; Hassabis, D. Human-level control through deep reinforcement learning. , v. 518, p. 529-533, fev. 2015.

MOERLAND, T. M.; BROEKENS, J.; JONKER, C. M. Emotion in reinforcement learning agents and robots: a survey. Machine Learning, Springer, v. 107, n. 2, p. 443-480, 2018.

MOLlAND, A.; TURNOCK, S. Marine Rudders and Control Surfaces. [S.l.: s.n.], 2007. ISBN 9780750669443.

MUNIN. Research in maritime autonomous systems project results and technology potentials. 2016. 12 p. Disponível em:〈http://www.unmanned-ship.org/munin/wpcontent/uploads/2016/02/MUNIN-final-brochure.pdf $\rangle$.

NAKAYAMA, M.; KAMIO, T.; MITSUBORI, K.; TANAKA, T.; FUJISAKA, H. Reinforcement learning based search for ships' courses controlled by safety. In: Proc. of NOLTA. [S.l.: s.n.], 2014. p. 28-31.

NG, A. Y.; COATES, A.; DIEL, M.; GANAPATHI, V.; SCHUlTE, J.; TSE, B.; BERGER, E.; LIANG, E. Autonomous inverted helicopter flight via reinforcement learning. In: Experimental Robotics IX. [S.l.]: Springer, 2006. p. 363-372.

PERERA, L. P. Autonomous ship navigation under deep learning and the challenges in colregs. In: AMERICAN SOCIETY OF MECHANICAL ENGINEERS. ASME 2018 37th International Conference on Ocean, Offshore and Arctic Engineering. [S.l.], 2018. p. V11BT12A005-V11BT12A005.

RAK, A.; SC, M. Reinforcement Learning in Discrete and Continuous Domains Applied to Ship Trajectory Generation. v. 19, n. 74, p. 31-36, 2012. ISSN 12332585.

RANDL $\varnothing \mathrm{V}$, J.; ALSTR $\varnothing \mathrm{M}$, P. Learning to Drive a Bicycle using Reinforcement Learning and Shaping. Proceedings of the International Conference on Machine Learning (ICML), p. 463-471, 1998.

RIEDMILLER, M. Neural fitted q iteration-first experiences with a data efficient neural reinforcement learning method. In: SPRINGER. European Conference on Machine Learning. [S.l.], 2005. p. 317-328. 
RUSSELL, S.; NORVIG, P. Artificial Intelligence: A Modern Approach. 3rd. ed. Upper Saddle River, NJ, USA: Prentice Hall Press, 2009. ISBN 0136042597, 9780136042594.

SCHAUL, T.; HORGAN, D.; GREGOR, K.; SILVER, D. Universal value function approximators. In: International conference on machine learning. [S.l.: s.n.], 2015. p. $1312-1320$.

SCHULMAN, J.; MORITZ, P.; LEVINE, S.; JORDAN, M.; ABBEEL, P. Highdimensional continuous control using generalized advantage estimation. arXiv preprint arXiv:1506.02438, 2015.

SCHULMAN, J.; WOLSKI, F.; DHARIWAL, P.; RADFORD, A.; KLIMOV, O. Proximal policy optimization algorithms. arXiv preprint arXiv:170\%.0634\%, 2017.

SPEIGHT R.; STRANNIGAN, D. Culturally aware simulation. In: International Conference on Marine Simulation and Ship Manoeuvrability. [S.l.: s.n.], 2015.

STAMENKOVICH, M. An application of artificial neural networks for autonomous ship navigation through a channel. In: IEEE PLANS 92 Position Location and Navigation Symposium Record. Monterey, CA, USA: IEEE, 1992. p. 346-352. ISBN 0-7803-0468-3. ISSN 87568470. Disponível em: 〈http://ieeexplore.ieee.org/document/185865/ $\rangle$.

SUTTON, R. S.; BARTO, A. G. Reinforcement Learning: An Introduction. MIT Press, 2018. (Adaptive Computation and Machine Learning). ISBN 9780262039246. Disponível em:〈https://mitpress.mit.edu/books/reinforcement-learning-second-edition〉.

SZEPESVÁRI, C. Algorithms for reinforcement learning. Synthesis lectures on artificial intelligence and machine learning, Morgan \& Claypool Publishers, v. 4, n. 1, p. 1-103, 2010.

TANIGAWA, T.; KAMIO, T.; MITSUBORI, K.; TANAKA, T.; FUJISAKA, H.; HAEIWA, K. Modified multi-agent reinforcement learning system to find ships' courses. IEICE Proceedings Series, The Institute of Electronics, Information and Communication Engineers, v. 2, n. 487, 2013.

TUYEN, L. P.; LAYEK, A.; VIEN, N. A.; CHUNG, T. Deep reinforcement learning algorithms for steering an underactuated ship. In: $201^{77}$ IEEE International Conference on Multisensor Fusion and Integration for Intelligent Systems (MFI). [S.l.: s.n.], 2017. p. 602-607.

Van Den Boogaard, M.; FEYS, A.; OVERBEEK, M.; Le Poole, J.; HEKKENBERG, R. Control concepts for navigation of autonomous ships in ports. 10Th Symposium on High-Performance Marine Vehicles, n. October, 2016.

WANG, C.; ZHANG, X.; LI, R.; DONG, P. Path planning of maritime autonomous surface ships in unknown environment with reinforcement learning. In: SPRINGER. International Conference on Cognitive Systems and Signal Processing. [S.1.], 2018. p. 127-137.

WATKINS, C. J. C. H.; DAYAN, P. Q-learning. Machine Learning, v. 8, n. 3, p. 279-292, May 1992. ISSN 1573-0565. Disponível em:〈https://doi.org/10.1007/BF00992698〉. 
WU, X.; CHEN, H.; CHEN, C.; ZHONG, M.; XIE, S.; GUO, Y.; FUJITA, H. The autonomous navigation and obstacle avoidance for usvs with anoa deep reinforcement learning method. Knowledge-Based Systems, Elsevier, p. 105201, 2020.

ZACCONE, R.; MARTELLI, M. A random sampling based algorithm for ship path planning with obstacles. In: Proceedings of the International Ship Control Systems Symposium (iSCSS). [S.l.: s.n.], 2018. v. 2, p. 4.

ZHANG, X.; WANG, C.; LIU, Y.; CHEN, X. Decision-making for the autonomous navigation of maritime autonomous surface ships based on scene division and deep reinforcement learning. Sensors, Multidisciplinary Digital Publishing Institute, v. 19, n. 18, p. 4055, 2019.

ZHAO, L.; ROH, M.-I. Colregs-compliant multiship collision avoidance based on deep reinforcement learning. Ocean Engineering, Elsevier, v. 191, p. 106436, 2019.

ZHOU, C.; GU, S.; WEN, Y.; DU, Z.; XIAO, C.; HUANG, L.; ZHU, M. The review unmanned surface vehicle path planning: Based on multi-modality constraint. Ocean Engineering, Elsevier, v. 200, p. 107043, 2020.

ZHU, H.; GUPTA, A.; RAJESWARAN, A.; LEVINE, S.; KUMAR, V. Dexterous manipulation with deep reinforcement learning: Efficient, general, and low-cost. In: IEEE. 2019 International Conference on Robotics and Automation (ICRA). [S.l.], 2019. p. 3651-3657. 